\title{
Total Synthesis of the Boron Containing Ion Carrier Antibiotic Macrodiolide Tartrolon B
}

\author{
Johann Mulzer* and Markus Berger \\ Institut für Organische Chemie der Universität Wien, Währinger Strasse 38, A-1090 Wien, Austria
}

\section{Supporting Information}

General: Fourier transform infrared spectra were calibrated on an internal standard and are reported in wavenumbers $\left(\mathrm{cm}^{-1}\right) .{ }^{1} \mathrm{H}$ - and ${ }^{13} \mathrm{C}$-NMR spectra were taken on 250, 400 and 600 $\mathrm{MHz}$ NMR machines. Proton chemical shifts are reported in $\delta$, using the residual $\mathrm{CHCl}_{3}$ as internal reference $(7.26 \mathrm{ppm})$. Carbon chemical shifts are reported in $\delta$, using $\mathrm{CDCl}_{3}$ as an internal standard (77.0 ppm). Mass spectra were obtained under electron impact (EI), field desorption (FD), field ionization and fast atom bombardment (FAB) conditions. Fast atom bombardment high resolution spectra were calibrated with CsI. Most FAB samples were doped with CsI and 3-nitrobenzoyl alcohol was used as matrix. A cesium gun was used as ion source with settings of $20 \mu \mathrm{A}$ and $20 \mathrm{KV}$.

Commercially available chemicals were used without further purification. Where appropriate, reactions were performed in flame-dried glassware under an argon atmosphere.

Ether $\left(\mathrm{Et}_{2} \mathrm{O}\right)$, tetrahydrofuran (THF) and toluene were freshly distilled from sodium benzophenone ketyl under an argon atmosphere. Dichloromethane $\left(\mathrm{CH}_{2} \mathrm{Cl}_{2}\right)$, diisopropylamine $\left(\operatorname{Pr}_{2} \mathrm{NH}\right)$, triethylamine $\left(\mathrm{Et}_{3} \mathrm{~N}\right)$, Hünig's base $\left({ }^{\mathrm{i}} \mathrm{Pr}_{2} \mathrm{EtN}\right)$ and dimethyl sulfoxide (DMSO) were distilled from calcium hydride. Hexanes and ethyl acetate (EtOAc) were distilled to remove higher boiling fractions.

Silica gel 60 (230-400 mesh) was used for column chromatography. Precoated aluminium sheets 60 were used for analytical thin layer chromatography (TLC). Compounds were visualised with UV light, p-anisaldehyde stain, or phosphomolybdic acid in EtOH. 


\section{Typical Procedures (TPs)}

\section{TP 1. Swern Oxidation}

Oxalyl chloride $(1 \mathrm{~mL}, 11 \mathrm{mmol})$ in dichloromethane $(25 \mathrm{~mL})$ was treated with DMSO $(1.7$ $\mathrm{mL}, 25 \mathrm{mmol})$ at $-78^{\circ} \mathrm{C}$. The mixture was stirred for $15 \mathrm{~min}$ at $-78^{\circ} \mathrm{C}$, then the alcohol $(10$ mmol) in dichlorometane $(10 \mathrm{~mL})$ was added and the mixture was stirred at $-78^{\circ} \mathrm{C}$ for $1 \mathrm{~h}$. $\mathrm{NEt}_{3}(5.5 \mathrm{~mL}, 40 \mathrm{mmol})$ was added and the cooling was removed. The completeness of the reaction was checked via TLC. Water $(25 \mathrm{~mL})$ was added, the organic layer was separated, washed with brine, dried over magnesium sulfate and concentrated under reduced pressure. The residue was purified by chromatography.

\section{TP 2. DiBAL-H Reduction of Esters}

The ester $(10 \mathrm{mmol})$ in diethylether $(25 \mathrm{~mL})$ was cooled to $-90^{\circ} \mathrm{C}$ and treated dropwise with a $1.2 \mathrm{M}$ solution $(10 \mathrm{~mL}, 12 \mathrm{mmol}) \mathrm{DIBAL}-\mathrm{H}$ in toluene at a temperature of below $-85^{\circ} \mathrm{C}$. The mixture was stirred for $2 \mathrm{~h}$ at $-90^{\circ} \mathrm{C}$. A satd. aqueous solution of tartaric acid $(10 \mathrm{~mL})$ was added, the organic layer was separated, washed with brine, dried over magnesium sulfate and concentrated under reduced pressure. The residue was purified by chromatography.

\section{TP 3. Hydrogenation with Raney Nickel}

The substrate in methanol $(3 \mathrm{~mL} / \mathrm{mmol})$ and Raney nickel $(0.2 \mathrm{~g} / \mathrm{mmol})$ were placed in a Parr apparatus and hydrogenated under 6 bar of hydrogen until no more hydrogen was absorbed. The hydrogen was then replaced by argon and the solution was filtered, concentrated under reduced pressure and the residue was purified by chromatography.

\section{TP 4. Desilylation with Dowex 50}

The silyl ether in THF $(5 \mathrm{ml} / \mathrm{mmol})$ was treated with DOWEX $(1 \mathrm{~g} / \mathrm{mmol})$. The mixture was stirred at room temperature until the starting material was consumed. After filtration the ion exchange resin was washed with ether, and the organic phase was concentrated under reduced pressure. The residue was purified by chromatography.

\section{TP 5. Desilylation with aqueous HF in acetonitrile}


The silyl ether in acetonitrile $(2 \mathrm{~mL} / \mathrm{mmol})$ was placed into a polypropylene vessel and treated

dropwise with conc. aqueous $\mathrm{HF}(0.5 \mathrm{~mL} / \mathrm{mmol})$. The mixture was stirred at room temperature until the starting material was consumed. Ether was added and the reaction was quenched with satd. aqu. $\mathrm{NaHCO}_{3}$. The organic phase was separated, washed with brine, dried over magnesium sulfate, concentrated under reduced pressure and the residue was chromatographed.

\section{TP 6. OH-Protection with PMB}

The alcohol in cyclohexane/dichloromethane $4: 1(2.5 \mathrm{~mL} / \mathrm{mmol})$ was treated dropwise with 1.2 eq $p$-methoxybenzyltrichloracetamidate at room temperature. The mixture was cooled to $0^{\circ} \mathrm{C}$ and camphorsulfonic acid $(0.1 \mathrm{eq})$ was added. The mixtuere was stirred without cooling over night, filtered over celite and concentrated under reduced pressure. The residue was purified by chromatography.

\section{TP 7. Removal of PMB Group}

The PMB ether in dichloromethane/ 1M phosphate buffer $\mathrm{pH} 7(9: 1,5 \mathrm{~mL} / \mathrm{mmol})$ was treated with solid DDQ $(1.2 \mathrm{eq})$ and stirred at room temperature for $1 \mathrm{~h}$. After completion the reaction was quenched with satd. aqueous $\mathrm{NaHCO}_{3}$. The organic phase was washed with brine, dried over magnesium sulfate, concentrated under reduced pressure and chromatographed..

\section{Ethyl-(2E, 4S)-4-tert-Butyldiphenylsilyloxy-pent-2-enoate}

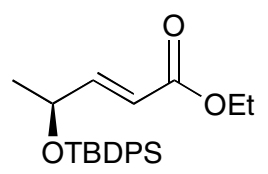

A suspension of sodium hydride $(80 \%, 1.68 \mathrm{~g}, 56 \mathrm{mmol})$ in THF $(30 \mathrm{~mL})$ was treated dropwise with carboxyethylmethylene diethylphosphonate $(12.8 \mathrm{~g}, 57 \mathrm{mmol})$ in THF $(20 \mathrm{~mL})$ for $2 \mathrm{~h}$. (2S)-2-tert-butyldimethylsilyl-oxypropanal (10 g, $28 \mathrm{mmol})$ in THF (100 mL) was added and the mixture was stirred at $22^{\circ} \mathrm{C}$ for $16 \mathrm{~h}$. After addition of saturated aqueous sodium fluoride the product was extracted with ether, filtered, dried over $\mathrm{NaSO}_{4}$ and purified 
by column chromatography (hexanes: ether $=5: 1$ ) to give ethyl- $(2 E, 4 S)$-4-tertbutyldiphenylsilyloxy-pent-2-enoate $(8.0 \mathrm{~g}, 75 \%)$ as a colorless oil. Anal calcd. For $\mathrm{C}_{23} \mathrm{H}_{30} \mathrm{O}_{3} \mathrm{Si}: \mathrm{C}, 72.21 ; \mathrm{H}, 7.90$; found: C, 72.09; H, 8.12. ${ }^{1} \mathrm{H}-\mathrm{NMR}\left(250 \mathrm{MHz} ; \mathrm{CDCl}_{3}\right): \delta$ $[\mathrm{ppm}]=7.73-7.66(\mathrm{~m}, 4 \mathrm{H}), 7.45-7.33(\mathrm{~m}, 6 \mathrm{H}), 6.94(\mathrm{dd}, J=15.1,4.7 \mathrm{~Hz}, 1 \mathrm{H}), 6.06(\mathrm{~d}, J=$ $15.1 \mathrm{~Hz}, 1 \mathrm{H}), 4.42(\mathrm{qt}, J=6.1,4.7 \mathrm{~Hz}, 1 \mathrm{H}), 4.09(\mathrm{q}, J=7.5 \mathrm{~Hz}, 2 \mathrm{H}), 1.23(\mathrm{t}, J=7.1 \mathrm{~Hz}$, 3H), 1.05 (s, 9H), 1.06 (d, $J=6.1 \mathrm{~Hz}, 3 \mathrm{H}) .{ }^{13} \mathrm{C}-\mathrm{NMR}\left(63 \mathrm{MHz} ; \mathrm{CDCl}_{3}\right): \delta[\mathrm{ppm}]=166.4$, $151.4,135.8,134.1,129.5,129.4,127.5,127.4,119.1,68.6,60.2,26.9,23.2,19.2,14.2 . \mathrm{MS}$ (EI, 70eV, 200 $\left.{ }^{\circ} \mathrm{C}\right): \mathrm{m} / \mathrm{z}=383$ [M-1] (<1), 327 [M-57] (100), 281 (4), 227 (58), 199 (82), $183(43), 139(32) ;[\alpha]_{\mathrm{D}}^{20}=-40.0^{\circ}\left(\mathrm{c}=2.0, \mathrm{CHCl}_{3}\right)$. IR ( Film) $1 / \lambda\left[^{\mathrm{cm}-1}\right]=3071,2964$, 2931, 2858, 1737, 1473, 1427, 1376, 1265, 1177, 1135, 1110, 1026, 997, 822, 740, 701.

\section{Ethyl-(4S)-tert- Butyldiphenylsilyloxy-pentanoate}

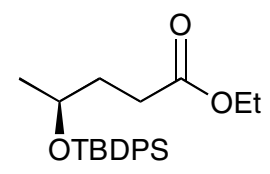

Ethyl-(2E, 4S)-4-tert-butyldiphenylsilyloxy-pent-2-enoate $(8.0 \mathrm{~g}, 21 \mathrm{mmol})$ were hydrogenated with Raney nickel (TP 3) to give ethyl-(4S)-tert- butyldiphenylsilyloxypentanoate $(6.7 \mathrm{~g}, 83 \%)$ as a colorless oil after chromatography (hexane/EtOAc $10: 1, \mathrm{R}_{\mathrm{f}}$ =0.42). Anal.calcd.for $\mathrm{C}_{23} \mathrm{H}_{32} \mathrm{O}_{3} \mathrm{Si}: \mathrm{C}, 71.83 ; \mathrm{H}, 8.39$; found: $\mathrm{C}, 71.99 ; \mathrm{H}, 8.45$. ${ }^{1} \mathrm{H}-\mathrm{NMR}$ $\left(250 \mathrm{MHz} ; \mathrm{CDCl}_{3}\right): \delta[\mathrm{ppm}]=7.73-7.66(\mathrm{~m}, 4 \mathrm{H}), 7.45-7.33(\mathrm{~m}, 6 \mathrm{H}), 4.09(\mathrm{q}, J=7.5 \mathrm{~Hz}$, 2H), 3.96 (qt, $J=6.1,5.5 \mathrm{~Hz}, 1 \mathrm{H}), 2.38(\mathrm{t}, J=7.5 \mathrm{~Hz}, 1 \mathrm{H}), 2.37(\mathrm{t}, J=7.5 \mathrm{~Hz}, 1 \mathrm{H}), 1.78(\mathrm{~m}$, 2H), $1.23(\mathrm{t}, J=7.1 \mathrm{~Hz}, 3 \mathrm{H}), 1.05(\mathrm{~s}, 9 \mathrm{H}), 1.04(\mathrm{~d}, J=6.1 \mathrm{~Hz}, 3 \mathrm{H}) .{ }^{13} \mathrm{C}-\mathrm{NMR}(63 \mathrm{MHz}$; $\left.\mathrm{CDCl}_{3}\right): \delta[\mathrm{ppm}]=173.8,135.8,134.1,129.5,129.4,127.5,127.4,68.6,60.2,34.2,30.0$, 27.0, 23.0, 19.2, 14.2. MS (EI, 70eV, $\left.200^{\circ} \mathrm{C}\right): \mathrm{m} / \mathrm{z}=382$ [M-2] $(<1), 325$ [M-57] (48), 227 (37), 199 (100), $183(23) .[\alpha]_{\mathrm{D}}^{20}=-6.6^{\circ}\left(\mathrm{c}=2.1, \mathrm{CHCl}_{3}\right)$. IR ( Film) $1 / \lambda\left[^{\mathrm{cm}-1}\right]=3071,2959$, 2931, 2858, 1719, 1659, 1472, 1428, 1367, 1272, 1152, 1111, 1054, 977, 822, 740, 702.

\section{Ethyl- (4S)-tert- Butyldiphenylsilyloxy-pentanoate}

was prepared analogously.

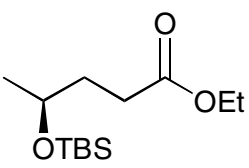


Anal calcd. for $\mathrm{C}_{23} \mathrm{H}_{32} \mathrm{O}_{3} \mathrm{Si}$ : C, 71.83; H, 8.39; found: C, 71.99 ;H, 8.45. ${ }^{1} \mathrm{H}-\mathrm{NMR}(250 \mathrm{MHz}$; $\left.\mathrm{CDCl}_{3}\right): \delta[\mathrm{ppm}]=7.73-7.66(\mathrm{~m}, 4 \mathrm{H}), 7.45-7.33(\mathrm{~m}, 6 \mathrm{H}), 6.94(\mathrm{dd}, J=15.1,4.7 \mathrm{~Hz}, 1 \mathrm{H})$, $6.06(\mathrm{~d}, J=15.1 \mathrm{~Hz}, 1 \mathrm{H}), 4.42(\mathrm{qt}, J=6.1,4.7 \mathrm{~Hz}, 1 \mathrm{H}), 4.09$ (q, $J=7.5 \mathrm{~Hz}, 2 \mathrm{H}), 1.23(\mathrm{t}, J=$ $7.1 \mathrm{~Hz}, 3 \mathrm{H}), 1.05(\mathrm{~s}, 9 \mathrm{H}), 1.06(\mathrm{~d}, J=6.1 \mathrm{~Hz}, 3 \mathrm{H})$;

(4S)-4-tert-Butyldimethylsilyloxypentanal (8a)

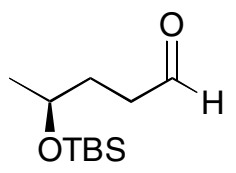

Ethyl-4(S)-4-tert-butyldimethylsilyloxypentanoate (10 g, $26 \mathrm{mmol})$ was reduced with DIBAL-H (TP 2) to give, after column chromatography (hexanes/ EtOAc $10: 1, \mathrm{R}_{\mathrm{f}}=0.39$ ) $8 \mathbf{a}(7.54 \mathrm{~g}, 90 \%)$ as a colorless oil. Anal.Calcd.for $\mathrm{C}_{11} \mathrm{H}_{24} \mathrm{O}_{2} \mathrm{Si}$ : C, 61,05; H, 11,18; found: C, 61,33; H, 10,94. ${ }^{1} \mathrm{H}-\mathrm{NMR}\left(250 \mathrm{MHz} ; \mathrm{CDCl}_{3}\right): \delta=9.78(\mathrm{t}, J=1.7 \mathrm{~Hz}, 1 \mathrm{H}) ; 3.87(\mathrm{tq}, J=7.1$, $6.2 \mathrm{~Hz}, 1 \mathrm{H}), 2.49$ (td, $J=7.3,1.7 \mathrm{~Hz}, 2 \mathrm{H}), 1.69$ (m, 2H), 1.14 (d, $J=6.2 \mathrm{~Hz}, 3 \mathrm{H}) ; 0.88$ (s, 9H,), 0.05 (s, 3H), 0.04 (s, 3H). ${ }^{13} \mathrm{C}-\mathrm{NMR}\left(63 \mathrm{MHz} ; \mathrm{CDCl}_{3}\right): \delta[\mathrm{ppm}]=202.7,67.5,40.1$, 31.7, 25.8, 23.6, 18.0, -4.4, -4.8. MS (EI, 70eV, 50 $\mathrm{C}): \mathrm{m} / \mathrm{z}=217$ [M+1] (2), 199 [M-OH] (4), 175 (48), 159 [M-57] (45), 157 (72), $141(8) ; 75(100) .[\alpha]_{\mathrm{D}}^{20}=+20,8^{\circ}\left(\mathrm{c}=2.0 ; \mathrm{CHCl}_{3}\right)$. IR (Film) $1 / \lambda\left[\mathrm{cm}^{-1}\right]=2957,2931,2858,1712,1463,1377,1256,1138,1091,1031,837$, 775.

(4S)-4-tert-Butyldiphenylsilyloxypentanal (8b)

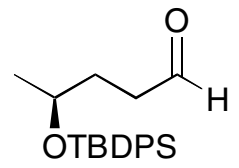

Ethyl (4S)-tert- Butyldiphenylsilyloxy-pentanoate $(22.0 \mathrm{~g}, 57.3 \mathrm{mmol})$ was reduced analogously to give $\mathbf{8 b}$ (18.3 g (94\%) after chromatography (hexanes/EtOAc, 5:1, $\mathbf{R}_{\mathrm{f}}=0.43$ ) as a colorless oil. Anal.calcd. for $\mathrm{C}_{21} \mathrm{H}_{28} \mathrm{O}_{2} \mathrm{Si}$ : C, 74.07; H, 8.29; found: C, 74.17; H, 8.31. ${ }^{1} \mathrm{H}-\mathrm{NMR}\left(250 \mathrm{MHz} ; \mathrm{CDCl}_{3}\right): \delta=9.69(\mathrm{t}, J=1.7 \mathrm{~Hz}, 1 \mathrm{H}), 7.73-7.66(\mathrm{~m}, 4 \mathrm{H}), 7.45-7.33(\mathrm{~m}$, $6 \mathrm{H}), 3.96(\mathrm{qt}, J=6.1,5.5 \mathrm{~Hz}, 1 \mathrm{H}), 2.49(\mathrm{td}, J=7.5,1.7 \mathrm{~Hz}, 2 \mathrm{H}), 1.78(\mathrm{~m}, 2 \mathrm{H}), 1.08(\mathrm{~d}, J=$ 
$6.1 \mathrm{~Hz}, 3 \mathrm{H}) ; 1.07(\mathrm{~s}, 9 \mathrm{H}) .{ }^{13} \mathrm{C}-\mathrm{NMR}\left(63 \mathrm{MHz} ; \mathrm{CDCl}_{3}\right): \delta[\mathrm{ppm}]=202.4,135.8,134.0$, $129.5,129.4,127.6,127.5,68.4,39.6,31.3,26.9,23.0,19.2 . \mathrm{MS}\left(\mathrm{EI}, 70 \mathrm{eV}, 50^{\circ} \mathrm{C}\right): \mathrm{m} / \mathrm{z}=$ 339 [M-1] (3), 321 [M-19] (2), 283 [M-57] (48), 221 (53), 199 (100), 181 (17), 139 (38);

$[\alpha]_{\mathrm{D}}^{20}=-1.5^{\circ}\left(\mathrm{c}=2.0, \mathrm{CHCl}_{3}\right) . \mathrm{IR}($ Film $) 1 / \lambda\left[\mathrm{cm}^{-1}\right]=3066,2957,2934,2853,1726,1472$, $1432,1381,1142,1107,1041,1005,817,701$.

(5S)-1,1-Dibromo-5-tert-butyldimethylsilyloxy-1-hexene

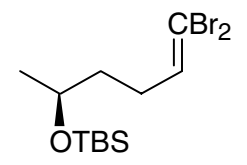

Triphenylphosphine (20.2 g , $77 \mathrm{mmol})$ and powdered zinc $(5.0 \mathrm{~g}, 77 \mathrm{mmol}, 2 \mathrm{eq})$ were suspended in dichloromethane $(200 \mathrm{~mL})$ and treated dropwise at $0^{\circ} \mathrm{C}$ with tetrabromomethane $(25.5 \mathrm{~g}, 77 \mathrm{mmol})$. The mixture was stirred at ambient temperature for 16 $\mathrm{h}$, until the zinc had dissolved. Pyridine (10 drops) and then $8 \mathbf{8}(8.38 \mathrm{~g}, 38.5 \mathrm{mmol}, 1 \mathrm{eq}$.$) in$ dichloromethane $(100 \mathrm{~mL})$ was added and the mixture was stirred for $2 \mathrm{~h}$. Then hexane (200 $\mathrm{mL}$ ) was added and the mixture was filtered and concentrated under reduced pressure, diluted with hexanes, filtered, and evaporated under reduced pressure.

${ }^{1} \mathrm{H}-\mathrm{NMR}\left(250 \mathrm{MHz} ; \mathrm{CDCl}_{3}\right): \delta[\mathrm{ppm}]=6.41(\mathrm{t}, J=7.2 \mathrm{~Hz}, 1 \mathrm{H}), 3.81(\mathrm{tq}, J=6.2,5.9 \mathrm{~Hz}$, 1H), 2.0-2.23 (m, 2H), 1.48-1.62 (m, 2H), 1.14 (d, J = 5.9 Hz, 3H), 0.89 (s, 9H), 0.05 (s, $6 \mathrm{H}) .{ }^{13} \mathrm{C}-\mathrm{NMR}\left(63 \mathrm{MHz} ; \mathrm{CDCl}_{3}\right): \delta[\mathrm{ppm}]=138.7,88.6,67.8,37.4,29.5,25.9,23.7,18.1$, 4.3, -4.7. HRMS (EI) calcd. for: $\left[\mathrm{M}\left(2^{79} \mathrm{Br}\right)-\mathrm{C}_{4} \mathrm{H}_{9}\right]: 312.9259$; found: [M(2 $\left.\left.\left.{ }^{79} \mathrm{Br}\right)\right)-\mathrm{C}_{4} \mathrm{H}_{9}\right]$ :312.9249. MS (EI, 70eV, $\left.60^{\circ} \mathrm{C}\right): \mathrm{m} / \mathrm{z}=373\left[\mathrm{M}\left(2^{81} \mathrm{Br}\right)-1\right](<1), 371\left[\mathrm{M}\left({ }^{79} \mathrm{Br} /{ }^{81} \mathrm{Br}\right)-1\right](<1)$, $369\left[\mathrm{M}\left(2^{79} \mathrm{Br}\right)-1\right](<1), 359\left[\mathrm{M}\left(2^{81} \mathrm{Br}\right)-15\right]$ (1), $357\left[\mathrm{M}\left({ }^{79} \mathrm{Br} /{ }^{81} \mathrm{Br}\right)-15\right]$ (2), $355\left[\mathrm{M}\left(2^{79} \mathrm{Br}\right)-15\right]$ (1), 317 [M(2 $\left.\left.2^{81} \mathrm{Br}\right)-57\right](32), 315\left[\mathrm{M}\left({ }^{79} \mathrm{Br} /{ }^{81} \mathrm{Br}\right)-57\right](64), 313$ [M(2 $\left.\left.2^{79} \mathrm{Br}\right)-57\right]$ (32), 201 (4), 199 (6), 197 (4), 161 (17), 159 (28), 157 (9), 141 (8), 139 (31), 137 (24), 97 (24), 80 (45), 79 (48), 75 (100). $[\alpha]_{\mathrm{D}}^{20}=+7.2^{\circ}\left(\mathrm{c}=2, \mathrm{CHCl}_{3}\right)$. IR (Film) $1 / \lambda\left[\mathrm{cm}^{-1}\right]=3307,2955,2930,2856$, $1771,1471,1463,1407,1378,1256,1135,1074,1051,837,808,775$.

\section{(5S)-1,1-Dibromo-5-tert-butyldiphenylsilyloxy-1-hexene}

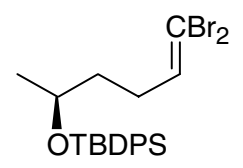


The compound was prepared analogously from $8 \mathbf{b}(7.5 \mathrm{~g}, 22.1 \mathrm{mmol})$. Chromatography (hexanes/EtOAc, 10:1, $\mathrm{R}_{\mathrm{f}}=0.61$ ) furnished $8.2 \mathrm{~g} \mathrm{(} 75 \%$ ) of the dibromide. Anal.calcd.for $\mathrm{C}_{22} \mathrm{H}_{28} \mathrm{OBr}_{2} \mathrm{Si}: \mathrm{C}, 53.24 ; \mathrm{H}, 5.69$; found: C, 53.01; H, 5.67. ${ }^{1} \mathrm{H}-\mathrm{NMR}\left(250 \mathrm{MHz} ; \mathrm{CDCl}_{3}\right): \delta$ $[\mathrm{ppm}]=7.66-7.73(\mathrm{~m}, 4 \mathrm{H}), 7.35-7.43(\mathrm{~m}, 6 \mathrm{H}), 6.25(\mathrm{t}, J=7.2 \mathrm{~Hz}, 1 \mathrm{H}), 3.88(\mathrm{tq}, J=6.2,5.9$ $\mathrm{Hz}, 1 \mathrm{H}), 2.00-2.23(\mathrm{~m}, 2 \mathrm{H}), 1.48-1.62(\mathrm{~m},, 2 \mathrm{H}), 1.11(\mathrm{~d}, J=6.2 \mathrm{~Hz}, 3 \mathrm{H}), 1.09(\mathrm{~s}, 9 \mathrm{H}) .{ }^{13} \mathrm{C}-$ $\operatorname{NMR}\left(63 \mathrm{MHz} ; \mathrm{CDCl}_{3}\right): \delta[\mathrm{ppm}]=138.5,135.9,135.8,134.5,134.0,129.6,129.5,127.5$, 127.3, 88.6, 68.7, 37.1, 28.9, 26.9, 23.0, 19.1. MS (EI, 70eV, 300 $\left.{ }^{\circ} \mathrm{C}\right): \mathrm{m} / \mathrm{z}=441\left[\mathrm{M}\left(2^{81} \mathrm{Br}\right)-\right.$ 57] (11), 439 [M( $\left.\left({ }^{79} \mathrm{Br} /{ }^{81} \mathrm{Br}\right)-57\right]$ (21), 437 [M( $\left.\left.2^{79} \mathrm{Br}\right) 57\right]$ (11), 263 (26), 261 (25), 213 (57), 199 (100), $183(32), 181(35), 79(26), 77(31) .[\alpha]_{\mathrm{D}}^{20}=-10.2^{\circ}\left(\mathrm{c}=2.0, \mathrm{CHCl}_{3}\right) . \mathrm{IR}(\mathrm{Film})$ $1 / \lambda\left[\mathrm{cm}^{-1}\right]=3071,3049,2963,2931,2856,1726,1472,1462,1427,1378,1136,1112$, $1044,998,822,741,703$.

\section{(5S) 5-tert-Butyldimethylsilyloxy-1-hexyne (9a)}

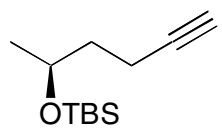

Variation A: Magnesium turnings (1.4 g), and (5S)-1,1-dibromo-5-tertbutyldimethylsilyloxy-1-hexene $(1 \mathrm{~g})$ in THF $(10 \mathrm{~mL})$ were treated dropwise with $0.5 \mathrm{~mL} 1,2-$ dibromoethane until the reaction started. Then additional dibromohexene (10.3 g, total 30.33 mmol) in THF (50 mL) was added slowly to keep the mixture under gentle reflux. Then the mixture was filtered and chromatographed (hexanes/EtOAc 5:1; $\left.\mathrm{R}_{\mathrm{f}}=0.69\right)$ to furnish 9a (6.1 $\mathrm{g}, 95 \%)$ as a colorless oil.

Variation B: (5S)-1,1-Dibromo-5-tert-butyldimethyl-silyloxy-1-hexene (22.3 g, $60 \mathrm{mmol})$ in THF $(200 \mathrm{~mL})$ was treated at $-50^{\circ} \mathrm{C}$ with $1.6 \mathrm{M}$ buthyl lithium in hexane $(7 \mathrm{~mL}, 11 \mathrm{mmol})$ The mixture was stirred $1 \mathrm{~h}$ at $-40^{\circ} \mathrm{C}$, and for $30 \mathrm{~min}$ at room temperature. The reaction was quenched with sat. aqu. $\mathrm{NH}_{4} \mathrm{Cl}(50 \mathrm{~mL})$ and extracted with ether. The ether phase was separated, dried $\left(\mathrm{MgSO}_{4}\right)$ evaporated and chromatographed to give 9a $(12.0 \mathrm{~g}, 94 \%)$ as a colorless oil.

Anal. Calcd. for $\mathrm{C}_{12} \mathrm{H}_{24} \mathrm{OSi}$ : C, 67.86; H, 11.39; found: C, 68.03; H, 11.22. ${ }^{1} \mathrm{H}-\mathrm{NMR}(250$ $\left.\mathrm{MHz} ; \mathrm{CDCl}_{3}\right): \delta=3.93(\mathrm{tq}, J=6.3,5.9 \mathrm{~Hz}, 1 \mathrm{H}), 2.24(\mathrm{dt}, J=7.2 \mathrm{~Hz}, 2.6 \mathrm{~Hz}, 2 \mathrm{H}), 1.92(\mathrm{t}$, $J=2.6 \mathrm{~Hz}, 1 \mathrm{H}), 1.62(\mathrm{td}, J=7.2, \mathrm{~Hz}, 6.3 \mathrm{H}), 1.14(\mathrm{~d}, J=5.9 \mathrm{~Hz}, 3 \mathrm{H}), 0.89(\mathrm{~s}, 9 \mathrm{H}), 0.06(\mathrm{~s}$, $6 \mathrm{H}) .{ }^{13} \mathrm{C}-\mathrm{NMR}\left(63 \mathrm{MHz} ; \mathrm{CDCl}_{3}\right): \delta[\mathrm{ppm}]=84.7,68.3,67.2 ., 38.4,29.9,26.0,23.8,18.2$, - 
4.2, -4.6. MS: (EI, $\left.70 \mathrm{eV}, 30^{\circ} \mathrm{C}\right) \mathrm{m} / \mathrm{z}=211$ [M-1] (<1), 197 [M-15] (3), 159 (12), 155 [M57] (68), 111 ( 8), 75 (100). $[\alpha]_{\mathrm{D}}^{20}=+41.7^{\circ}\left(\mathrm{c}=2, \mathrm{CHCl}_{3}\right)$. IR (Film) $1 / \lambda\left[\mathrm{cm}^{-1}\right]=3314$, 2956, 29302858 1727, 1462, 1374, 1255, 1137, 1088, 1037, 997, 838, 775, 738.

(5S)-5-tert-Butyldiphenylsilyloxy-1-hexyne (9b)

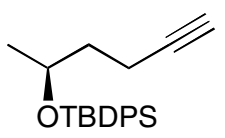

Variation A was used to convert (5S)-1,1-dibromo-5-tert-butyldiphenylsilyloxy-1-hexene (8.0 g, $16.1 \mathrm{mmol})$ into 9b (4.9 g, $91 \%)$. Anal. Calcd. for $\mathrm{C}_{22} \mathrm{H}_{28} \mathrm{OSi}: \mathrm{C}, 78.51 ; \mathrm{H}, 8.39$; found: C, 78.26; H, 8.30. ${ }^{1} \mathrm{H}-\mathrm{NMR}\left(250 \mathrm{MHz} ; \mathrm{CDCl}_{3}\right)$ : $\delta=7.66-7.73(\mathrm{~m}, 4 \mathrm{H}), 7.35-7.45(\mathrm{~m}$, $6 \mathrm{H}), 3.99(\mathrm{qt}, J=6.2,5.9 \mathrm{~Hz}, 1 \mathrm{H}), 2.27(\mathrm{dt}, J=7.4 \mathrm{~Hz}, 2.6 \mathrm{~Hz}, 2 \mathrm{H}), 1.87$ (t, $J=2.6 \mathrm{~Hz}$, $1 \mathrm{H}), 1.61-1.80(\mathrm{~m}, 2 \mathrm{H}), 1.07$ (s, 9H), $1.06(\mathrm{~d}, J=6.2 \mathrm{~Hz}, 3 \mathrm{H}) .{ }^{13} \mathrm{C}-\mathrm{NMR}\left(63 \mathrm{MHz} ; \mathrm{CDCl}_{3}\right)$ : $\delta[\mathrm{ppm}]=135.9,134.7,134.0,129.5,129.4,127.5,127.3,84.5,68.4,68.0 ., 38.1,26.9,23.0$, 19.3, 14.5. MS: (EI, $\left.70 \mathrm{eV}, 200^{\circ} \mathrm{C}\right) \mathrm{m} / \mathrm{z}=337$ [M+1] (2), 279 [M-57] (100), 259 (12), 237 (16), $199(61), 183(14), 84(48) .[\alpha]_{\mathrm{D}}^{20}=+4.9^{\circ}\left(\mathrm{c}=2, \mathrm{CHCl}_{3}\right)$. IR (Film) $1 / \lambda\left[\mathrm{cm}^{-1}\right]=3309$, 3071, 2963, 2931, 2893, 2858, 1472, 1137, 1111, 1086, 1035, 996, 822, 741, 703.

\section{$(3 R / S)(8 S)-8-t e r t-B u t y l d i m e t h y l s i l y l o x y-n o n-1-e n-4-y n-9-o l ~(10 a)$}

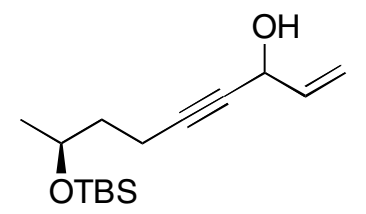

9a $(10 \mathrm{~g}, 29.7 \mathrm{mmol})$ in $\mathrm{THF}(60 \mathrm{~mL})$ at $-78^{\circ} \mathrm{C}$ were treated with $14 \mathrm{~mL}(35 \mathrm{mmol}) 2.5 \mathrm{M} n$ $\mathrm{BuLi}$ in hexane. The mixture was stirred for $20 \mathrm{~min}$ and then warmed to $20^{\circ} \mathrm{C}$. $\mathrm{LiBr}(1.3 \mathrm{~g}$, $15 \mathrm{mmol}, 0.5 \mathrm{eq}$ ) was added and the mixture was stirred for $20 \mathrm{~min}$ to dissolve the $\mathrm{LiBr}$. Then the mixture was cooled to $-78^{\circ} \mathrm{C}$ and acrolein $(2.1 \mathrm{~g}, 37.5 \mathrm{mmol})$ in THF ( $10 \mathrm{~mL}$ ) was added over a period of $2 \mathrm{~h}$. After hydrolytic workup 10a (89\%) was isolated as a mixture of two diastereomers. Anal.calcd. for $\mathrm{C}_{15} \mathrm{H}_{28} \mathrm{O}_{2} \mathrm{Si}: \mathrm{C}, 67,11 ; \mathrm{H}, 10,51$; found: $\mathrm{C}, 67,41 ; \mathrm{H}$, 10,40. ${ }^{1} \mathrm{H}-\mathrm{NMR}\left(400 \mathrm{MHz} ; \mathrm{CDCl}_{3}\right): \delta[\mathrm{ppm}]=5.96(\mathrm{ddd}, J=17.0,10.3,5.2 \mathrm{~Hz}, 1 \mathrm{H}), 5.42$ $(\mathrm{d}, J=17.0 \mathrm{~Hz}, 1 \mathrm{H}), 5.18(\mathrm{~d}, J=10.3 \mathrm{~Hz}, 1 \mathrm{H}), 4.85$ (dd, $J=5.3,2.1 \mathrm{~Hz}, 1 \mathrm{H}) 3.90$ (tq, $J$ $=6.2,5.9 \mathrm{~Hz}, 1 \mathrm{H}), 2.29(\mathrm{td}, J=7.2,2.1 \mathrm{~Hz}, 2 \mathrm{H}), 1.87(\mathrm{~m}, 1 \mathrm{H}), 1.64(\mathrm{td}, J=7.2,5.9 \mathrm{~Hz}$, $2 \mathrm{H}), 1.13(\mathrm{~d}, J=6.2 \mathrm{~Hz}, 3 \mathrm{H}), 0.88(\mathrm{~s}, 9 \mathrm{H}) ; 0.06(\mathrm{~s}, 6 \mathrm{H})$. 
${ }^{13} \mathrm{C}-\mathrm{NMR}\left(63 \mathrm{MHz} ; \mathrm{CDCl}_{3}\right): \delta[\mathrm{ppm}]=137.7,115.9,87.1,79.1,67.1,63.4,38.3,25.9,23.7$, 18.1, 15.2, -4.3; -4.8. MS (EI, 70eV, 50 C) m/z = 267 [M-1] (1), 253 [M-15] (3), 211 (17), 169 (33), 159 (19), $119(100), 117(27), 103(17), 91(94), 7591) .[\alpha]_{\mathrm{D}}^{20}=+45^{\circ}(\mathrm{c}=2.0$; $\left.\mathrm{CHCl}_{3}\right) . \mathrm{IR}(\mathrm{Film}) 1 / \lambda\left[\mathrm{cm}^{-1}\right]=2942,2871,1727,1455,1442,1376,1352,1200,1160$, $1132,1115,1077,1034,912,870$.

\section{$(3 R / S, 8 S)-8$-tert-Butyldiphenylsilyloxy-non-1-en-4-yn-9-ol (10b)}

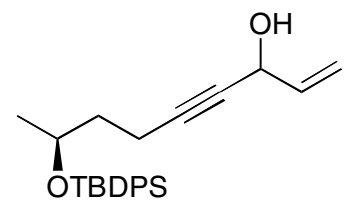

Analogously, 9b $(6.7 \mathrm{~g}, 22 \mathrm{mmol})$ was converted into 10b. After chromatography ( $n$ hexane/EtOAc, 3:1, $\left.\mathrm{R}_{\mathrm{f}}=0.27\right) \mathbf{1 0 b}(7.7 \mathrm{~g}, 89 \%)$ was obtained as a colorless oil. Anal. calcd. for $\mathrm{C}_{27} \mathrm{H}_{32} \mathrm{O}_{2} \mathrm{Si}: \mathrm{C}, 76.48 ; \mathrm{H}, 8.22$; found: C, 76.21; H, 8.23. ${ }^{1} \mathrm{H}-\mathrm{NMR}\left(400 \mathrm{MHz} ; \mathrm{CDCl}_{3}\right)$ : $\delta$ $[\mathrm{ppm}]=7.73-7.66(\mathrm{~m}, 4 \mathrm{H}), 7.45-7.33(\mathrm{~m}, 6 \mathrm{H}), 5.94(\mathrm{ddd}, J=17.0,10.3,5.2 \mathrm{~Hz}, 1 \mathrm{H}), 5.43$ (d, $J=17.0 \mathrm{~Hz}, 1 \mathrm{H}), 5.20(\mathrm{~d}, J=10.3 \mathrm{~Hz}, 1 \mathrm{H}), 4.83(\mathrm{~m}, 1 \mathrm{H}) 3.98(\mathrm{tq}, J=6.2,5.9 \mathrm{~Hz}, 1 \mathrm{H})$, $2.31(\mathrm{td}, J=7.4,2.1 \mathrm{~Hz}, 2 \mathrm{H}), 1.87(\mathrm{~m}, 1 \mathrm{H}), 1.61-1.80(\mathrm{~m}, 2 \mathrm{H}), 1.07$ (s, 9H), 1.06 (d, $J=6.2$ $\mathrm{Hz}, 3 \mathrm{H}) .{ }^{13} \mathrm{C}-\mathrm{NMR}\left(63 \mathrm{MHz} ; \mathrm{CDCl}_{3}\right): \delta[\mathrm{ppm}]=137.5,135.8,134.6,134.1,129.5,129.4$, $127.5,127.4,115.9,86.9,78.9,68.3,63.2,38.1,27.0,23.1,19.2,14.9 . \mathrm{MS}\left(\mathrm{EI}, 70 \mathrm{eV}, 70^{\circ} \mathrm{C}\right)$ $\mathrm{m} / \mathrm{z}=333$ [M-59] (25), 257 (6), 199 (100), $181(13), 159(11), 139(44), 119(40) .[\alpha]_{\mathrm{D}}^{20}=-$ $2.9^{\circ}\left(\mathrm{c}=2.1 ; \mathrm{CHCl}_{3}\right)$. IR (Film) $1 / \lambda\left[\mathrm{cm}^{-1}\right]=2942,2871,1727,1455,1442,1376,1352$, 1200, 1160, 1132, 1115, 1077, 1034, 912, 870.

Ethyl- (4E, 10S) 10-tert-Butyldimethylsilyloxyundec-4-en-6-ynoate (11a)

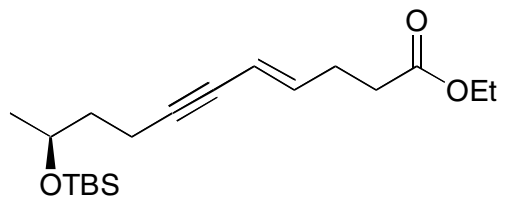

10a $(1.5 \mathrm{~g}, 43 \mathrm{mmol})$, triethylorthoacetate $(40 \mathrm{~mL})$ and propionic acid $(0.2 \mathrm{~mL})$ in xylenes $(200 \mathrm{~mL})$ were refluxed for $2 \mathrm{~h}$. Then the mixture was concentrated under reduced pressure, and the residue was chromatographed (hexanes/ethyl acetate $5: 1, \mathrm{R}_{\mathrm{f}}=0.50$ ) to give 11a ( $13.7 \mathrm{~g}, 94 \%)$ as a $6: 1-(E / Z)$-mixture, from which the $E$-isomer was separated by HPLC (2.5\% EtOAc in hexane; $\left.\mathrm{t}_{\mathrm{R}}=3.2 \mathrm{~min}\right)$. Anal. Calcd. for $\mathrm{C}_{19} \mathrm{H}_{34} \mathrm{O}_{3} \mathrm{Si}: \mathrm{C}, 67,40 ; \mathrm{H}, 10,12$; 
found C, 67.59; H, 9.95. ${ }^{1} \mathrm{H}-\mathrm{NMR}\left(250 \mathrm{MHz} ; \mathrm{CDCl}_{3}\right): \delta[\mathrm{ppm}]=6.01(\mathrm{dt}, J=15.7,6.7 \mathrm{~Hz}$, $1 \mathrm{H}), 5.50(\mathrm{dt}, J=15.7,2.0 \mathrm{~Hz}, 1 \mathrm{H}), 4.13(\mathrm{q}, J=7.1 \mathrm{~Hz}, 2 \mathrm{H}), 3.90(\mathrm{tq}, J=6.2,5.9 \mathrm{~Hz}, 1 \mathrm{H})$, 2.36-2.44 (m, 4H), 2.32 (td, $J=7.3,2.0 \mathrm{~Hz}, 2 \mathrm{H}), 1.59$ (td, $J=7.3,5.9 \mathrm{~Hz}, 2 \mathrm{H}), 1.25$ (t, $J$ $=7.1 \mathrm{~Hz}, 3 \mathrm{H}), 1.13(\mathrm{~d}, J=6.2 \mathrm{~Hz}, 3 \mathrm{H}), 0.88(\mathrm{~s}, 9 \mathrm{H}) ; 0.06(\mathrm{~s}, 6 \mathrm{H}) \cdot{ }^{13} \mathrm{C}-\mathrm{NMR}(63 \mathrm{MHz}$; $\left.\mathrm{CDCl}_{3}\right): \delta[\mathrm{ppm}]=172.3,140.2,111.2,89.1,78.7,66.9,60.2,38.3,33.3,28.0,25.7,23.6$, 17.9, 15.6, 14.1, -4.5, -4.9. MS (EI, 70eV, 30 $\left.{ }^{\circ} \mathrm{C}\right) \mathrm{m} / \mathrm{z}=323$ [M-15] (1); 281 [M-57] (16); 239 (12); 235 (38); $207(100), 119(24), 103(13), 91(17), 75(42) .[\alpha]_{\mathrm{D}}^{20}=+38.0^{\circ}(\mathrm{c}=2$; $\mathrm{CH}_{2} \mathrm{Cl}_{2}$ ). IR (Film) $1 / \lambda\left[^{\mathrm{cm}-1}\right]=2930 ; 2857 ; 1738 ; 1472 ; 1374 ; 1255 ; 1137 ; 1086 ; 1031$; $954 ; 837 ; 775$.

\section{Ethyl-(4E, 10S)-10-tert-butyldiphenylsilyloxyundec-4-en-6-ynoate (11b)}

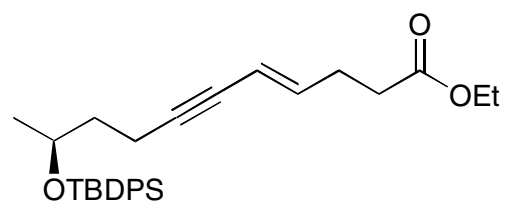

Analogously 10b $(5.1 \mathrm{~g}, 12.9 \mathrm{mmol})$ was converted into 11b. After chromatography (nhexane/EtOAc, 10:1, $\left.\mathrm{R}_{\mathrm{f}}=0.35\right) 5.3 \mathrm{~g}(88 \%)$ were obtained as a 6:1- $(\mathrm{E} / \mathrm{Z})$ mixture from which the $E$-isomer was isolated by HPLC $\left(2.5 \%\right.$ EtOAc in hexane; $\left.\mathrm{t}_{\mathrm{R}}=2.8 \mathrm{~min}\right)$. Anal. Calcd. for $\mathrm{C}_{29} \mathrm{H}_{38} \mathrm{O}_{3} \mathrm{Si}$ : C, 75.28; H, 8.28; found: C, 75.11; H, 8.39. ${ }^{1} \mathrm{H}-\mathrm{NMR}(400 \mathrm{MHz}$; $\left.\mathrm{CDCl}_{3}\right): \delta[\mathrm{ppm}]=7.73-7.66(\mathrm{~m}, 4 \mathrm{H}), 7.45-7.33(\mathrm{~m}, 6 \mathrm{H}), 6.35(\mathrm{dd}, J=15.0,11.1 \mathrm{~Hz}, 1 \mathrm{H})$, $5.92(\mathrm{dd}, J=11.0,10.9 \mathrm{~Hz}, 1 \mathrm{H}), 5.64(\mathrm{dt}, J=15.0,6.7 \mathrm{~Hz}, 1 \mathrm{H}), 5.34(\mathrm{dt}, J=10.9,7.4 \mathrm{~Hz}$, $1 \mathrm{H}), 4.14(\mathrm{q}, J=7.1 \mathrm{~Hz}, 2 \mathrm{H}), 3.98(\mathrm{qt}, J=6.2,6.0 \mathrm{~Hz}, 1 \mathrm{H}), 2.35-2.45(\mathrm{~m}, 6 \mathrm{H}), 2.05-2.33(\mathrm{t}$, $2 \mathrm{H}), 1.38-1.58(\mathrm{~m}, 2 \mathrm{H}), 1.25(\mathrm{t}, J=7.1 \mathrm{~Hz}, 3 \mathrm{H}), 1.07(\mathrm{~s}, 9 \mathrm{H}), 1.06(\mathrm{~d}, J=6.2 \mathrm{~Hz}, 3 \mathrm{H}) .{ }^{13} \mathrm{C}-$ $\operatorname{NMR}\left(63 \mathrm{MHz} ; \mathrm{CDCl}_{3}\right): \delta[\mathrm{ppm}]=172.5,140.4,135.8,134.7,134.0,129.5,129.4,127.5$, 127.3, 111.2, 89.2, 78.7, 68.4, 60.4, 38.3, 33.4, 28.1, 26.9, 23.1, 19.2, 15.5, 14.2. MS (EI, $\left.70 \mathrm{eV}, 50^{\circ} \mathrm{C}\right) \mathrm{m} / \mathrm{z}=406$ [M-56] (35); 405 [M-57] (100); 359 (12); 307 (20); 227 (11); 207 (44); $199(60) ; 139(11) .[\alpha]_{\mathrm{D}}^{20}=-14,4^{\circ}\left(\mathrm{c}=2.1, \mathrm{CHCl}_{3}\right)$. IR (Film) $1 / \lambda\left[^{\mathrm{cm}-1}\right]=2963,2931$, 2857, 1737, 1472, 1428, 1375, 1252, 1162, 1137, 1111, 1025, 953, 822, 702.

\section{Ethyl-(4E, 6Z, 10S)-10-tert-Butyldimethylsilyloxyundeca-4,6-dienoate (12a)}

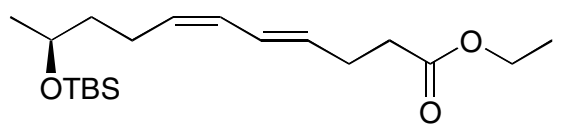


Zn powder $(13.7 \mathrm{~g})$ in ethanol $(20 \mathrm{~mL})$ was heated with 1,2-dibromoethane $(0.78 \mathrm{~mL})$, until a vigorous reaction occurred. The mixture was again treated with 1,2-dibromoethane $(0.78 \mathrm{~mL})$ and refluxed for $10 \mathrm{~min}$. The mixture was cooled to $50^{\circ} \mathrm{C}$ and $\mathrm{LiBr}(4.7 \mathrm{~g})$ and $\mathrm{CuBr}$ $(3.14 \mathrm{~g})$ in THF $(10 \mathrm{ml})$ were added within $3 \mathrm{~min} .11 \mathrm{a}(2.0 \mathrm{~g}, 5.9 \mathrm{mmol})$ in THF $(15 \mathrm{~mL})$ was added, the mixture was refluxed for $2 \mathrm{~h}$ and then quenched with sat. aqueous $\mathrm{NH}_{4} \mathrm{Cl}$. Normal workup furnished, after chromatography (hexanes/ethyl acetate $5: 1 ; R_{\mathrm{f}}=0.52$ ). 12a $(1.78 \mathrm{~g}, 89 \%)$ as a colorless oil. Anal. Calcd. for $\mathrm{C}_{19} \mathrm{H}_{36} \mathrm{O}_{3} \mathrm{Si}: \mathrm{C}, 67,01 ; \mathrm{H}, 10,65$; found: $\mathrm{C}$, 67,24; H, 10,46. ${ }^{1} \mathrm{H}-\mathrm{NMR}\left(400 \mathrm{MHz} ; \mathrm{CDCl}_{3}\right): \delta[\mathrm{ppm}]=6.35(\mathrm{dd}, J=15.0,11.1 \mathrm{~Hz}, 1 \mathrm{H})$, $5.92(\mathrm{dd}, J=11.0,10.9 \mathrm{~Hz}, 1 \mathrm{H}), 5.64(\mathrm{dt}, J=15.0,6.7 \mathrm{~Hz}, 1 \mathrm{H}), 5.34(\mathrm{dt}, J=10.9,7.4 \mathrm{~Hz}$, $1 \mathrm{H}), 4.14(\mathrm{q}, J=7.1 \mathrm{~Hz}, 2 \mathrm{H}), 3.81$ (qt, $J=6.1,6.0 \mathrm{~Hz}, 1 \mathrm{H}), 2.35-2.45$ (m, 4H), 2.05-2.33 (m, 2H), 1.38-1.58 (m, 2H), $1.25(\mathrm{t}, J=7.1 \mathrm{~Hz}, 3 \mathrm{H}), 1.13(\mathrm{~d}, J=6.1 \mathrm{~Hz}, 3 \mathrm{H}), 0.89$ (s, 9H), $0.06(\mathrm{~s}, 6 \mathrm{H}) .{ }^{13} \mathrm{C}-\mathrm{NMR}\left(63 \mathrm{MHz} ; \mathrm{CDCl}_{3}\right): \delta[\mathrm{ppm}]=172.8,131.8,130.6,128.2,126.6,68.0$, 60.2, 39.6, 34.0, 28.1, 25.8, 23.9, 23.7, 18.0, 14.2, -4.5, -4.8. MS (EI, 70eV, 30 $\left.{ }^{\circ} \mathrm{C}\right) \mathrm{m} / \mathrm{z}=$ 325 [M-15] (4); 283 [M-57] (100); 237 (15); 208 (36); 163 (19); 145 (12); 121 (40); 103 (15); 93 (25); 79 (22); 75 (42). $[\alpha]_{\mathrm{D}}^{20}=+24,1^{\circ}\left(\mathrm{c}=2 ; \mathrm{CHCl}_{3}\right)$. IR (Film) $1 / \lambda\left[\mathrm{cm}^{-1}\right]=2957$, 2930, 2857, 1739, 1463, 1373, 1302, 1255, 1162, 1137, 1095, 1037, 950, 884, 836, 775.

\section{Ethyl- (4E, 6Z, 10S)-10-tert-Butyldiphenylsilyloxyundeca-4,6-dienoate (12b)}

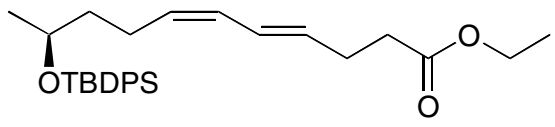

Analogously, 11b (1.20 g, $2.6 \mathrm{mmol})$ furnished 12b (1.13 g, $94 \%)$ after chromatography (hexanes/EtOAc, $10: 1, \mathrm{R}_{\mathrm{f}}=0.37$ ). Anal. Calcd. for $\mathrm{C}_{29} \mathrm{H}_{40} \mathrm{O}_{3} \mathrm{Si}: \mathrm{C}, 74.95 ; \mathrm{H}, 8.68$; found: $\mathrm{C}$, 75.19; H, 8.78. ${ }^{1} \mathrm{H}-\mathrm{NMR}\left(400 \mathrm{MHz} ; \mathrm{CDCl}_{3}\right): \delta[\mathrm{ppm}]=7.73-7.66(\mathrm{~m}, 4 \mathrm{H}), 7.45-7.33(\mathrm{~m}$, $6 \mathrm{H}), 6.35(\mathrm{dd}, J=15.0,11.1 \mathrm{~Hz}, 1 \mathrm{H}), 5.92(\mathrm{dd}, J=11.0,10.9 \mathrm{~Hz}, 1 \mathrm{H}), 5.64(\mathrm{dt}, J=15.0$, $6.7 \mathrm{~Hz}, 1 \mathrm{H}), 5.34$ (dt, $J=10.9,7.4 \mathrm{~Hz}, 1 \mathrm{H}), 4.14$ (q, $J=7.1 \mathrm{~Hz}, 2 \mathrm{H}), 3.89$ (qt, $J=6.1,5.9$ $\mathrm{Hz}, 1 \mathrm{H}), 2.35-2.45(\mathrm{~m}, 4 \mathrm{H}), 2.05-2.33(\mathrm{~m}, 2 \mathrm{H}), 1.38-1.58(\mathrm{~m}, 2 \mathrm{H}), 1.25(\mathrm{t}, J=7.1 \mathrm{~Hz}, 3 \mathrm{H})$, $1.09(\mathrm{~d}, J=6.1 \mathrm{~Hz}, 3 \mathrm{H}), 1.05(\mathrm{~s}, 9 \mathrm{H}) .{ }^{13} \mathrm{C}-\mathrm{NMR}\left(63 \mathrm{MHz} ; \mathrm{CDCl}_{3}\right): \delta[\mathrm{ppm}]=172.9,136.3$, 135.3, 134.9, 134.9, 132.3, 131.1, 129.9, 129.8, 127.9, 127.8, 127.0, 69.2, 60.3, 38.7, 34.5, 28.6, 27.5, 24.0, 23.6, 19.7, 14.4. MS (EI, 70eV, 70 $\mathrm{C}) \mathrm{m} / \mathrm{z}=408$ [M-56] (30), 407 [M-57] (100), 243 (13), 227 (19), 209 (16), 199 (17), 183 (30), 159 (22), 139 (23), 121 (38), 105 
(21). $[\alpha]_{\mathrm{D}}^{20}=+24,1^{\circ}\left(\mathrm{c}=2 ; \mathrm{CHCl}_{3}\right) . \mathrm{IR}\left(\right.$ Film) $1 / \lambda\left[\mathrm{cm}^{-1}\right]=3070,2963,2930,2857,1739$, $1473,1373,1248,1162,1134,1111,1036,950,822,703$.

(4E, 6Z, 10S)-10-tert-Butyldimethylsilyloxyundeca-4,6-dienal (4a)

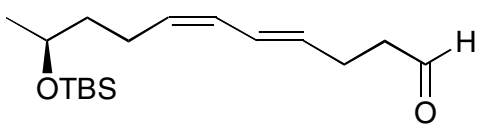

12a (1.0 g (2.9 mmol) was reduced with DIBAL-H (TP 2) to give, after chromatography (hexanes /ethyl acetate $\left.5: 1, \mathrm{R}_{\mathrm{f}}=0,43\right) \mathbf{4} \mathbf{a}(0.82 \mathrm{~g}, 93 \%)$ as a colorless oil. Anal.calcd. for $\mathrm{C}_{17} \mathrm{H}_{32} \mathrm{O}_{2} \mathrm{Si}$ : C, 68,86; H, 10,88; found: C, 69,13; H, 10,60. ${ }^{1} \mathrm{H}-\mathrm{NMR}$ (400 MHz; $\mathrm{CDCl}_{3}$ ): $\delta[\mathrm{ppm}]=9.88(\mathrm{t}, J=1.5 \mathrm{~Hz}, 1 \mathrm{H}), 6.36(\mathrm{dd}, J=15.1,11.0 \mathrm{~Hz}, 1 \mathrm{H}), 5.92(\mathrm{dd}, J=11.0$, $10.8 \mathrm{~Hz}, 1 \mathrm{H}), 5.64(\mathrm{dt}, J=15.1,6.6 \mathrm{~Hz}, 1 \mathrm{H}), 5.35$ (dt, $J=10.8,7.4 \mathrm{~Hz}), 3.82$ (tq, $J=6.1$ $\mathrm{Hz}, 1 \mathrm{H}), 2.24(\mathrm{dt}, J=7.2 \mathrm{~Hz}, J=2.6 \mathrm{~Hz}, 2 \mathrm{H}), 1.92(\mathrm{t}$, $J=2.6 \mathrm{~Hz}, 1 \mathrm{H}), 1.62(\mathrm{dt}, J=7.2, \mathrm{~Hz}, 2 \mathrm{H}), 1.13(\mathrm{~d}, J=6.1 \mathrm{~Hz}, 3 \mathrm{H}), 0.89(\mathrm{~s}, 9 \mathrm{H}), 0.06$ (s, $6 \mathrm{H}) .{ }^{13} \mathrm{C}-\mathrm{NMR}\left(63 \mathrm{MHz} ; \mathrm{CDCl}_{3}\right): \delta[\mathrm{ppm}]=201.9,131.7,131.2,128.3,127.1,68.3,43.6$, 39.8, 26.1, 25.5, 24.3, 23.9, 18.3, -4.1, -4.5. MS (EI, 70eV, 30 $\left.{ }^{\circ} \mathrm{C}\right) \mathrm{m} / \mathrm{z}=281[\mathrm{M}-15]$ (1), 239 [M-57] (59), 197 (7), 183 (7), 164 (43), 147 (16), 121 (100), 105 (19), 101 (24), 93 (97), 79 (89), 75 (72). $[\alpha]_{\mathrm{D}}^{20}=+25,5^{\circ}\left(\mathrm{c}=2.0 ; \mathrm{CHCl}_{3}\right)$. IR (Film) $1 / \lambda\left[^{\mathrm{cm}-1}\right]=2957 ; 2930 ; 2857$;

$1739 ; 1463 ; 1373 ; 1302 ; 1255 ; 1162 ; 1137 ; 1095 ; 1037 ; 950 ; 884,836 ; 775$.

\section{$(4 E, 6 Z, 10 S)$ 10-tert-Butyldiphenylsilyloxyundeca-4,6-dienal (4b)}

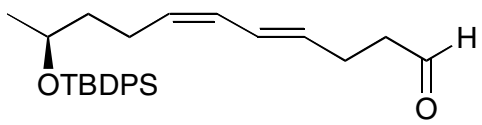

Analogously, 12b (1.13 g, mmol) gave 4b (1.0 g, 94\%) after chromatography ( $n$ hexane/EtOAc, 10:1, $\mathrm{R}_{\mathrm{f}}=0.33$ ). Anal. Calcd. for $\mathrm{C}_{27} \mathrm{H}_{36} \mathrm{O}_{2} \mathrm{Si}$ : C, 77.09; H, 8.63; found: $\mathrm{C}$, 76.82; H, 8.58. ${ }^{1} \mathrm{H}-\mathrm{NMR}\left(400 \mathrm{MHz} ; \mathrm{CDCl}_{3}\right): \delta[\mathrm{ppm}]=9.78(\mathrm{t}, J=1.4 \mathrm{~Hz}), 7.66-7.72(\mathrm{~m}$, 4H), 7.35-7.44 (m, 6H), $6.28(\mathrm{dd}, J=15.1,11.0 \mathrm{~Hz}, 1 \mathrm{H}), 5.87(\mathrm{dd}, J=11.0,10.7 \mathrm{~Hz}, 1 \mathrm{H})$, $5.62(\mathrm{dt}, J=15.1,7.1 \mathrm{~Hz}, 1 \mathrm{H}), 5.23(\mathrm{dt}, J=10.7,7.8 \mathrm{~Hz}), 3.87(\mathrm{qt}, J=6.2,5.9 \mathrm{~Hz}, 1 \mathrm{H}), 2.53$ (dt, $J=7.2,1.4 \mathrm{~Hz}, 2 H), 2.40(\mathrm{~m}, 2 \mathrm{H}), 2.17(\mathrm{~m}, 2 \mathrm{H}), 1.52(\mathrm{~m}, 2 \mathrm{H}), 1.07(\mathrm{~d}, J=6.1 \mathrm{~Hz}, 3 \mathrm{H})$, $1.06(\mathrm{~s}, 9 \mathrm{H}) .{ }^{13} \mathrm{C}-\mathrm{NMR}\left(63 \mathrm{MHz} ; \mathrm{CDCl}_{3}\right): \delta[\mathrm{ppm}]=201.7,135.9,135.8,134.8,134.5$, 131.4, 130.9, 129.5, 129.4, 128.0, 127.5, 127.4, 126.8, 69.2 43.3, 39.3, 27.0, 25.3, 23.6, 23.3, 19.3. MS (EI, 70eV, 200 $\mathrm{C}) \mathrm{m} / \mathrm{z}=421$ [M+1] (4), 363 [M-57] (21), 225 (22), 199 (68), 
183 (29), 121 (1), 121 (100), 105 (19), 101 (24), 93 (97), 79 (89), 75 (72). [ $\alpha]_{\mathrm{D}}^{20}=+25,5^{\circ}(\mathrm{c}$ $\left.=2.0 ; \mathrm{CHCl}_{3}\right)$. IR (Film) 1/ $\lambda\left[\mathrm{cm}^{-1}\right]=2957,2930,2857,1728,1589$ 1471, 1427, 1377, 1362, 1255, 1131, 1110, 1037, 998, 822.

\section{(1Z, 5S)-1-Iod-5-tert-butyldimethylsilyloxy-1-hexene (13)}

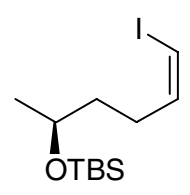

Iodomethyltriphenylphophonium iodide $(3.5 \mathrm{~g}, 66 \mathrm{mmol})$ in THF $(100 \mathrm{~mL})$ at $0^{\circ} \mathrm{C}$ was treated dropwise with 1.5 M NaHMDS in THF(45 mL). After 1 min the mixture was cooled to $-78^{\circ} \mathrm{C}$ and $(4 S)$-4-tert-Butyldimethylsilyloxypentanal $(1.42 \mathrm{~g}, 66 \mathrm{mmol})$ in THF $(150 \mathrm{~mL})$ was added. The mixture was stirred for $15 \mathrm{~min}$ at $-78^{\circ} \mathrm{C}$ and allowed to warm up for $30 \mathrm{~min}$. Hexane $(60 \mathrm{~mL})$ was added, the mixture was filtered and evaporated to dryness under reduced pressure. The residue was purified by chromatography (hexanes/EtOAc $10: 1$ ) to give $13(1.34 \mathrm{~g}, 60 \%)$ as a 7:1-E/Z-mixture. ${ }^{1} \mathrm{H}-\mathrm{NMR}\left(250 \mathrm{MHz} ; \mathrm{CDCl}_{3}\right): \delta[\mathrm{ppm}]=6.18$ (dt, $J=9.1,7.5 \mathrm{~Hz}, 1 \mathrm{H}), 6.17(\mathrm{~d}, J=9.1 \mathrm{~Hz}, 1 \mathrm{H}), 3.81$ (tq, $J=6.2,5.9 \mathrm{~Hz}, 1 \mathrm{H}), 2.0-2.23$ (m, 2H), 1.48-1.62 ( m, 2H) $1.14(\mathrm{~d}, J=5.9 \mathrm{~Hz}, 3 \mathrm{H}), 0.89(\mathrm{~s}, 9 \mathrm{H}), 0.05(\mathrm{~s}, 6 \mathrm{H}) .{ }^{13} \mathrm{C}-\mathrm{NMR}(63$ $\left.\mathrm{MHz}_{\mathrm{CDCl}}\right): \delta[\mathrm{ppm}]=141.2,82.2,68.0,37.7,31.3,25.9,23.7,18.1,-4.3,-4.7$; MS: (EI, 70eV, 20 $\left.{ }^{\circ} \mathrm{C}\right) \mathrm{m} / \mathrm{z}=440$ [M](2), 325 [M-15] (3),283 [M-57] (59),229 (21), 185 (22), 81 (15), 75 (100), 99 (67). HRMS (EI, $70 \mathrm{eV}$ ) calcd. for $\mathrm{C}_{8} \mathrm{H}_{16} \mathrm{OISi}$ :283.0015; found: 283.0016. $[\alpha]_{\mathrm{D}}^{20}=+18.5^{\circ}\left(\mathrm{c}=2.0 ; \mathrm{CHCl}_{3}\right)$. IR (Film) $1 / \lambda\left[\mathrm{cm}^{-1}\right]=2956,2929,2857,1609$, 1471, 1374, 1462, 1361, 1294, 1255, 1135, 1084, 1037, 1005, 836, 808, 776.

\section{1-Trityloxybut-3-yne}

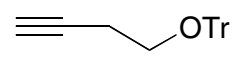

But-3-yn-1-ol (2.0 g, $28.5 \mathrm{mmol})$ in pyridine $(2 \mathrm{~mL})$ and dichloromethane $(70 \mathrm{~mL})$ was treated with molecular sieves $(20 \mathrm{~g})$ and tritylchloride $(8.3 \mathrm{~g}, 30 \mathrm{mmol})$ at $0^{\circ} \mathrm{C}$. The mixture was stirred at room temperature for $12 \mathrm{~h}$. Filtration and chromatography (hexanes /EtOAc 5 : 1) furnished 1-trityloxy-but-3-yne (8.27 g, 93\%) as an amorphous solid. ${ }^{1} \mathrm{H}-\mathrm{NMR}(250 \mathrm{MHz}$; $\left.\mathrm{CDCl}_{3}\right): \delta[\mathrm{ppm}]=7.42-7.48(\mathrm{~m}, 6 \mathrm{H}), 7.20-7.33(\mathrm{~m}, 9 \mathrm{H}), 3.25(\mathrm{t}, J=6.7 \mathrm{~Hz}, 2 \mathrm{H}), 2.52(\mathrm{dt}, J$ $=6.7,2.5 \mathrm{~Hz}, 2 \mathrm{H}), 2.04(\mathrm{t}, \mathrm{J}=3.1 \mathrm{~Hz}, 1 \mathrm{H}) .{ }^{13} \mathrm{C}-\mathrm{NMR}\left(63 \mathrm{MHz} ; \mathrm{CDCl}_{3}\right): \delta[\mathrm{ppm}]=144.0$, 128.7, 127.8, 127.0, 86.7, 81.6, 69.2, 62.0, 20.0. MS: (EI, 70eV, 50) $\mathrm{m} / \mathrm{z}=312$ [M] (19), 
284 (4), 243 (100), 236 (58), 165 (46), 105 (63), 83 (29). IR (Film) 1/ $\left[\mathrm{cm}^{-1}\right]=3289,2928$, 2880, 1594, 1488, 1446, 1209, 1155, 1082, 1029, 999, 740, 705.

\section{(3E)-1-Trityloxybut-3-entributylstannane (14)}

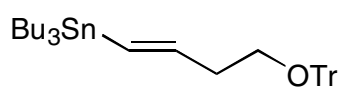

1-Trityloxy-but-3-yne $(8.10 \mathrm{~g}, 26 \mathrm{mmol})$ was heated with tri-n-butylstannane ( $30 \mathrm{mmol})$ and AIBN (500 mg) to $90^{\circ} \mathrm{C}$ for $5 \mathrm{~h}$. The product was purified by chromatography (hexanes/EtOAc $\left.10: 1 \mathrm{R}_{\mathrm{f}}=0.68\right)$ to give $14(11.3 \mathrm{~g}, 72 \%)$ as a colorless oil. ${ }^{1} \mathrm{H}-\mathrm{NMR}(250$ $\left.\mathrm{MHz} ; \mathrm{CDCl}_{3}\right): \delta[\mathrm{ppm}]=7.42-7.48(\mathrm{~m}, 6 \mathrm{H}), 7.20-7.33(\mathrm{~m}, 9 \mathrm{H}), 6.05(\mathrm{~m}, 2 \mathrm{H}), 3.17(\mathrm{t}, J=$ $6.7 \mathrm{~Hz}, 2 \mathrm{H}), 2.48(\mathrm{~m}, 2 \mathrm{H}), 1.45-1.58(\mathrm{~m}, 6 \mathrm{H}), 2.25-1.40(\mathrm{~m}, 6 \mathrm{H}), 0.85-0.95(\mathrm{~m}, 15 \mathrm{H}) .{ }^{13} \mathrm{C}-$ $\operatorname{NMR}\left(63 \mathrm{MHz} ; \mathrm{CDCl}_{3}\right): \delta[\mathrm{ppm}]=146.1,144.4,129.9$, 128.7, 127.7, 126.8, 86.3, 63.1, 38.6, 29.1, 27.3, 13.7, 9.4; MS: (EI, 70eV, 100 C) m/z =440 [M](2), 325 [M-15] (3),283 [M-57] (59),229 (21), 185 (22), 81 (15), 75 (100), 99 (67). HRMS (EI, $70 \mathrm{eV)} \mathrm{calcd.} \mathrm{for:}$ $\mathrm{C}_{31} \mathrm{H}_{39}$ OSn: 547.2022; found: 547.2043. IR (Film) $1 / \lambda\left[\mathrm{cm}^{-1}\right]=2932,2858,1428,1378$, 1214, 1137, 1110, 1025, 823, 703.

\section{(4E, 6Z, 10S)-10-tert-Butyldimethylsilyloxy-undeca-4,6-dien-1-tritylether (15)}

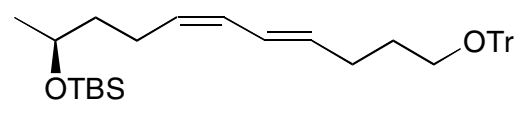

13 (280 mg, $0.82 \mathrm{mmol})$ and $14(495 \mathrm{mg}, 0.82 \mathrm{mmol})$ in DMF (5 mL) were treated with $\mathrm{Pd}(\mathrm{dba})_{3}(85 \mathrm{mg}, 0.08 \mathrm{mmol}), \mathrm{AsPh}_{3}(98 \mathrm{mg}, 0.3 \mathrm{mmol})$ and $\mathrm{CuI}(31 \mathrm{mg}, 0.16 \mathrm{mmol})$. The mixture was heated to $100{ }^{\circ} \mathrm{C}$ for $8 \mathrm{~h}$, diluted with water $(10 \mathrm{~mL})$ and extraceted with $\mathrm{Et}_{2} \mathrm{O}$ /hexanes 1:1. The organic layer was washed with brine, dried $\left(\mathrm{MgSO}_{4}\right)$ and concentrated under erduced pressure. The residue was chromatographed (hexanes/ethyl acetate 5:1; $\mathrm{R}_{\mathrm{f}}=$ $0.72)$ to give $15(350 \mathrm{mg}, 65 \%)$ as a colorless oil. ${ }^{1} \mathrm{H}-\mathrm{NMR}\left(400 \mathrm{MHz} ; \mathrm{CDCl}_{3}\right): \delta[\mathrm{ppm}]=$ 7.42-7.48 (m, 6H), 7.20-7.33 (m, 9H), 6.31 (dd, $J=15.0,10.9 \mathrm{~Hz}, 1 \mathrm{H}), 5.91$ (dd, $J=10.9$, $10.7 \mathrm{~Hz}, 1 \mathrm{H}), 5.62(\mathrm{dt}, J=15.0,6.8 \mathrm{~Hz}, 1 \mathrm{H}), 5.30(\mathrm{dt}, J=10.7,7.5 \mathrm{~Hz}), 3.82(\mathrm{tq}, J=6.1,5.9$ $\mathrm{Hz}, 1 \mathrm{H}), 3.09$ (t, $J=6.4 \mathrm{~Hz}, 2 \mathrm{H}), 2.08-2.35(\mathrm{~m}, 4 \mathrm{H}), 1.66-1.78(\mathrm{~m}, 2 \mathrm{H}), 1.43-1.55(\mathrm{~m}, 2 \mathrm{H})$, $1.14(\mathrm{~d}, J=5.9 \mathrm{~Hz}, 3 \mathrm{H}), 0.90(\mathrm{~s}, 9 \mathrm{H}), 0.06(\mathrm{~s}, 6 \mathrm{H}) .{ }^{13} \mathrm{C}-\mathrm{NMR}\left(63 \mathrm{MHz} ; \mathrm{CDCl}_{3}\right): \delta[\mathrm{ppm}]=$ 144.5, 134.1, 129.8, 128.7, 128.6, 127.7, 126.8, 125.8, 68.2, 63.0, 39.7, 29.9, 29.7, 25.9, 24.1, 23.7, 18.2, -4.3, -4.7. MS: (EI, 70eV, 20 C) m/z = $540[\mathrm{M}](<1), 331(<1), 297(5), 243$ 
(100), 165 (85), 105 (35). HRMS (EI, $70 \mathrm{eV)}$ calcd. for $\mathrm{C}_{36} \mathrm{H}_{48} \mathrm{O}_{2} \mathrm{Si}$ : 540.3424; found:

540.3424. $[\alpha]_{\mathrm{D}}^{20}=+13.8^{\circ}\left(\mathrm{c}=2.0 ; \mathrm{CHCl}_{3}\right)$. IR (Film) $1 / \lambda\left[\mathrm{cm}^{-1}\right]=2932,2858,1428,1378$, 1214, 1137, 1110, 1025, 823, 703 .

Conversion into aldehyde $4 a$. The trityl ether (300 $\mathrm{mg}, 5.5 \mathrm{mmol}$ ) was treated with a mixture of formic acid $(5 \mathrm{~mL})$ and ether $(10 \mathrm{~mL})$ at room temperature for $1 \mathrm{~h}$. The mixture was neutralized with $2 \mathrm{~N} \mathrm{NaOH}$ and then $5 \mathrm{~mL}$ of $2 \mathrm{~N} \mathrm{NaOH}$ was added and the mixture was stirred at room temperature for $30 \mathrm{~min}$. The product was extracted with ether, the organic layer was washed with brine, dried over magnesium sulfate and concentrated under reduced pressure. The crude alcohol was oxidized under Swern conditions (TP 1) to give aldehyde 4a (132 mg, $80 \%)$, identical in all respects with the material obtained above.

\section{Methyl -(2R)-2-Methyl-3-p-methoxybenzyloxy-propanoate (16)}

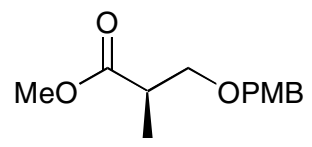

Commercially available methyl-(2R)-3-hydroxy-2-methylpropionate (16.5 g, $139 \mathrm{mmol})$ was converted (TP 6) into the PMB ether with p-methoxybenzyl-trichloracetimidate $(47.1 \mathrm{~g}, 167$ $\mathrm{mmol}$ ) to give after chromatography (hexanes/EtOAc $\left.5: 1 ; \mathrm{R}_{\mathrm{f}}=0.39\right) \mathbf{1 6}(29.5 \mathrm{~g}, 89 \%)$ as a colorless oil. Anal. calcd. for $\mathrm{C}_{13} \mathrm{H}_{18} \mathrm{O}_{4}$ : C, 65.55; H, 7.61; found: $\mathrm{C}, 65.73 ; \mathrm{H}, 7.55 .{ }^{1} \mathrm{H}-$ $\operatorname{NMR}\left(250 \mathrm{MHz} ; \mathrm{CDCl}_{3}\right): \delta[\mathrm{ppm}]=7.23(\mathrm{dt}, J=8.7,2.5 \mathrm{~Hz}, 2 \mathrm{H}), 6.87(\mathrm{dt}, J=8,7,2.5 \mathrm{~Hz}$, 2H), 4.44 (s, 2H), 3.79 (s, 3H), 3.68 (s, 3H), 3.63 (dd, J = 9.2, 7.3Hz, 1H), 3.45 (dd, J = 9.2, 5.9, 1H), $2.76(\mathrm{dqd}, \mathrm{J}=7.3,7.1,5.9 \mathrm{~Hz}, 1 \mathrm{H}), 1.16(\mathrm{~d}, \mathrm{~J}=7.1 \mathrm{~Hz}, 3 \mathrm{H}) .{ }^{13} \mathrm{C}-\mathrm{NMR}(63 \mathrm{MHz}$; $\left.\mathrm{CDCl}_{3}\right): \delta[\mathrm{ppm}]=175.3,159.1,130.2129 .2,113.7,72.7,71.6,55.2,51.7,40.1,14.0 . \mathrm{MS}$ (EI, $\left.70 \mathrm{eV}, 100^{\circ} \mathrm{C}\right): \mathrm{m} / \mathrm{z}=238$ [M] (18), 151(7), 137 (88), 121 (100), 229 (19), 211 (43), 121 (100). $[\alpha]_{\mathrm{D}}^{20}=-8.6^{\circ}\left(\mathrm{c}=1.96 ; \mathrm{CHCl}_{3}\right)$. IR (Film) $1 / \lambda\left[\mathrm{cm}^{-1}\right]=2951,2860,1739,1670 ; 1513$; $1461,1363,1301,1248,1174,1090,1034,820$.

\section{(2R)-3-p-Methoxybenzyloxy-2-methyl-propan-methoxymethylamide (17)}<smiles>COCC(C)C(=O)N(C)OC</smiles> 
16 ( $2.1 \mathrm{~g}, 8.8 \mathrm{mmol})$ and $\mathrm{Me}(\mathrm{MeO}) \mathrm{NH} \cdot \mathrm{HCl}(1.4 \mathrm{~g}, 1.41 \mathrm{mmol})$ in THF $(20 \mathrm{~mL})$ were treated at $-20^{\circ} \mathrm{C}$ with $2 \mathrm{M}$ isopropylmagnesiumchloride Lösung in $\mathrm{Et}_{2} \mathrm{O}(9 \mathrm{~mL}, 18 \mathrm{mmol})$. The mixture was stirred at $-10^{\circ} \mathrm{C}$ for 30 min and then worked up as usual to give after chromatography (hexanes/ethyl acetate $1: 1\left(\mathrm{R}_{\mathrm{f}}=0.36\right) \mathbf{1 7}(1.52 \mathrm{~g}, 65 \%)$ as a colorless oil. Anal. calcd. for $\mathrm{C}_{14} \mathrm{H}_{21} \mathrm{NO}_{4}$ : C, 62.90; H, 7.92; N, 5.24; found: C, 63.11; H, 7.70; N, 5.03. ${ }^{1} \mathrm{H}-\mathrm{NMR}\left(250 \mathrm{MHz} ; \mathrm{CDCl}_{3}\right): \delta[\mathrm{ppm}]=7.23(\mathrm{dt}, J=8.7,2.3 \mathrm{~Hz}, 2 \mathrm{H}), 6.85(\mathrm{dt}, J=8,7$, $2.3 \mathrm{~Hz}, 2 \mathrm{H}), 4.48(\mathrm{~d}, \mathrm{~J}=11.8 \mathrm{~Hz}, 1 \mathrm{H}), 4.40(\mathrm{~d}, \mathrm{~J}=11.8 \mathrm{~Hz}, 1 \mathrm{H}), 3.79(\mathrm{~s}, 3 \mathrm{H}), 3.68(\mathrm{~s}, 3 \mathrm{H})$, $3.68(\mathrm{dd}, \mathrm{J}=8.9,8.0 \mathrm{~Hz}, 1 \mathrm{H}), 3.39(\mathrm{dd}, \mathrm{J}=8.9,5.9,1 \mathrm{H}), 3.15-3.32(\mathrm{~m}, 1 \mathrm{H}), 1.10(\mathrm{~d}, \mathrm{~J}=$ $6.9 \mathrm{~Hz}, 3 \mathrm{H}) ;{ }^{13} \mathrm{C}-\mathrm{NMR}\left(63 \mathrm{MHz} ; \mathrm{CDCl}_{3}\right): \delta[\mathrm{ppm}]=159.1,130.5,129.1,113.7,72.9,72.3$, 61.5, 55.2, 35.9, 14.2. MS: (EI, $\left.70 \mathrm{eV}, 80^{\circ} \mathrm{C}\right) \mathrm{m} / \mathrm{z}=267$ [M] (1), 236 [M-31] (7), 131 (16), $121(100), 100$.

HRMS cald. for: $\mathrm{C}_{14} \mathrm{H}_{21} \mathrm{NO}_{4}: 267.1471$; found: $267.1464 .[\alpha]_{\mathrm{D}}^{20}=-2.9^{\circ}\left(\mathrm{c}=2.0 ; \mathrm{CHCl}_{3}\right)$. IR (Film) $1 / \lambda\left[\mathrm{cm}^{-1}\right]=3306,2968,2937,2861,2838,1660,1612 ; 1513 ; 1465,1420,1387$, $1302,1248,1175,1099,1034,994,820$.

\section{(2R)-1-tert-Butyldimethylsilyloxy-4,4-dibrom-2-methyl-but-3-ene}

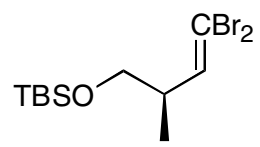

18 (4.0 g, $20 \mathrm{mmol})$ was converted into the dibromide as described to give after chromatography (hexanes/EtOAc $\left.5: 1 ; \mathrm{R}_{\mathrm{f}}=0,62\right)(2 R)$-1-tert-butyldimethylsilyloxy-4,4dibromo-2-methyl-but-3-ene $(4.6 \mathrm{~g}, 65 \%)$ as a colorless oil.

${ }^{1} \mathrm{H}-\mathrm{NMR}\left(400 \mathrm{MHz} ; \mathrm{CDCl}_{3}\right): \delta[\mathrm{ppm}]=6.27(\mathrm{~d}, \mathrm{~J}=9.1 \mathrm{~Hz}, 1 \mathrm{H}, 3.48(\mathrm{~d}, \mathrm{~J}=7.5,2 \mathrm{H}), 2.55$ $(\mathrm{dtq}, \mathrm{J}=9.1,7.5 \mathrm{~Hz}, 1 \mathrm{H}), 1.02(\mathrm{~d}, \mathrm{~J}=6.9 \mathrm{~Hz}, 3 \mathrm{H}), 0.89$ (s, 9H), 0.04 (s, 6H). ${ }^{13} \mathrm{C}-\mathrm{NMR}(100.6$ $\left.\mathrm{MHz} ; \mathrm{CDCl}_{3}\right): \delta[\mathrm{ppm}]=141.4,88.4,66.0,41.1,25.8,18.3,15.6,-5.4 . \mathrm{MS}(\mathrm{EI}, 70 \mathrm{eV}$, $\left.30^{\circ} \mathrm{C}\right): \mathrm{m} / \mathrm{z}=303\left[\mathrm{M}\left(2^{81} \mathrm{Br}\right)-57\right](11), 301\left[\mathrm{M}\left({ }^{79} \mathrm{Br} /{ }^{81} \mathrm{Br}\right)-57\right](19), 299$ [M( $\left.\left.2^{79} \mathrm{Br}\right)-57\right]$ (11), 273 [M(2 $\left.\left.2^{81} \mathrm{Br}\right)-87\right](11), 271\left[\mathrm{M}\left({ }^{79} \mathrm{Br} /{ }^{81} \mathrm{Br}\right)-57\right](20), 269$ [M( $\left.\left.2^{79} \mathrm{Br}\right)-87\right]$ (13), 139 (39), 89 (71), $83(100) .[\alpha]_{\mathrm{D}}^{20}=-14.0^{\circ}\left(\mathrm{c}=0.6 ; \mathrm{CHCl}_{3}\right)$. IR (Film) $1 / \lambda\left[\mathrm{cm}^{-1}\right]=2955 ; 2930 ; 2886$; $2857 ; 1613 ; 1513 ; 1463 ; 1249 ; 1090 ; 1035 ; 836 ; 776$.

$(2 R, 6 R)$ 7-tert-Butyldimethylsilyloxy-1-p-methoxybenzyloxy-2,6-dimethyl-hept-4-yn-3one (19)

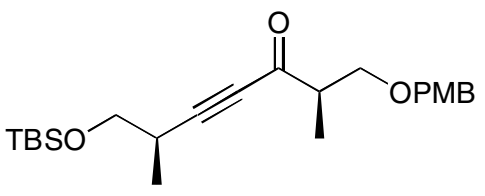


(2R)-1-tert-Butyldimethylsilyloxy-4,4-dibromo-2-methyl-but-3-ene (1.84 g, $5.1 \mathrm{mmol})$ in THF (25 mL) was treated dropwise at $-40^{\circ} \mathrm{C}$ with $1.6 \mathrm{M}$ butyl lithium $(7.0 \mathrm{~mL}, 11 \mathrm{mmol})$ in hexane. The mixture was stirred at $-40^{\circ} \mathrm{C}$ for $1 \mathrm{~h}$ and then at room temperature for $10 \mathrm{~min}$. The acetylide solution was cooled to $-78^{\circ} \mathrm{C}$ and treated dropwise over $15 \mathrm{~min}$ with 17 (1.36 $\mathrm{g}, 5.1 \mathrm{mmol})$ in $\mathrm{THF}(10 \mathrm{~mL})$. The mixture was slowly $(10 \mathrm{~min})$ warmed to $-20^{\circ} \mathrm{C}$, stirred for $1 \mathrm{~h}$ and then quenched with satd. $\mathrm{NH}_{4} \mathrm{Cl}(20 \mathrm{~mL})$. Usual workup yielded after chromatography (hexanes /EtOAc, $\left.3: 1, \mathrm{R}_{\mathrm{f}}=0.51\right) \mathbf{1 9}(1.65 \mathrm{~g}, 79 \%)$ as a colorless oil. Anal. calcd. for $\mathrm{C}_{23} \mathrm{H}_{36} \mathrm{O}_{4} \mathrm{Si}: \mathrm{C}, 68.27$; H, 8.97; found: C, 67.98; H, 8.67. ${ }^{1} \mathrm{H}-\mathrm{NMR}(400 \mathrm{MHz}$; $\left.\mathrm{CDCl}_{3}\right): \delta[\mathrm{ppm}]=7.22(\mathrm{~d}, J=8.1 \mathrm{~Hz}, 2 \mathrm{H}), 6.94(\mathrm{~d}, J=8,1 \mathrm{~Hz}, 2 \mathrm{H}), 4.42(\mathrm{~s}, 2 \mathrm{H}), 3.77(\mathrm{~s}, 3 \mathrm{H})$, 3.73 (dd, J=9.4, 7.3, 1H), 3.66 (dd, J=9.4, 5.9, 1H), 3.52 (dd, J=6.8, 2.0Hz, 1H), 3.49 (dd, $\mathrm{J}=5.5,2.0 \mathrm{~Hz}, 1 \mathrm{H}), 2.83$ (qdd, J=7.3, 6.9, 5.9Hz, 1H), 2.73 (qdd, J=6.9, 6.8, 5.5Hz, 1H), 1.19 $(\mathrm{d}, \mathrm{J}=6.9 \mathrm{~Hz}, 3 \mathrm{H}), 1.16$ (d, J=6.9Hz, 3H), 0.87 (s, 9H), 0.04 (s, 6H). ${ }^{13} \mathrm{C}-\mathrm{NMR}(63 \mathrm{MHz}$; $\left.\mathrm{CDCl}_{3}\right): \delta[\mathrm{ppm}]=189.8,159.2,130.2,129.2,113.7,96.7,80.2,72.8,71.0,66.2,55.2,48.9$, 29.5, 25.8, 18.3, 16.4, 13.3, -5.4, -5.5. MS (FI, 7kV, 3mA) m/z= 404 (76), 405 (27), 58 (100). $[\alpha]_{\mathrm{D}}^{20}=+13,6^{\circ}\left(\mathrm{c}=2 ; \mathrm{CHCl}_{3}\right)$. IR (Film) $1 / \lambda\left[\mathrm{cm}^{-1}\right]=2954 ; 2931 ; 2857 ; 2210 ; 1676$; $1613 ; 1513 ; 1464 ; 1302 ; 1249 ; 1097 ; 1037 ; 838 ; 778$.

$(2 R, 3 R / S, 6 R)$ 7-tertButyldimethylsilyloxy-1-p-methoxybenzyloxy -2,6-dimethyl-hept-4yn-3-ol (20 and its 3-epimer).

19 (404 mg, $1 \mathrm{mmol}$ ) was treated with commercially available $1 \mathrm{M}$ Alpine borane ${ }^{\circledR}$ solution $(3 \mathrm{~mL})$. The solution was sealed in a Teflon tube and kept in a high pressure apparatus at 6000 bar for $3 \mathrm{~d}$ at room temperature. The mixture was transferred into a glass flask and THF $(10 \mathrm{~mL})$ and propanal $(116 \mathrm{mg}, 2 \mathrm{mmol})$ were added and the mixture was stirred for $2 \mathrm{~h} .1 \mathrm{M}$ $\mathrm{NaOH}(1 \mathrm{~mL})$ and $30 \% \mathrm{H}_{2} \mathrm{O}_{2}(0.3 \mathrm{~mL})$ was added and the mixture was stirred for $3 \mathrm{~h}$ at room temperature. After neutralization with $\mathrm{NH}_{4} \mathrm{Cl}$ usual workup delivered after chromatography (hexanes/ ethyl acetate $3: 1) \mathbf{2 0}$ and its epimer (334 mg, $82 \%$ ) in a ratio of 9 $: 1$.

$(2 R, 3 S, 6 R)$ 7-tertButyldimethylsilyloxy-1-p-methoxybenzyloxy -2,6-dimethyl-hept-4yn-3-ol (20) 


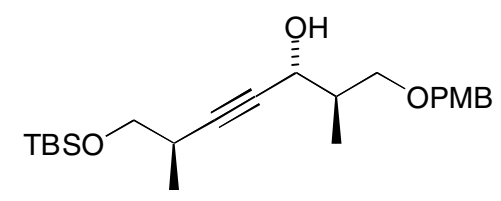

Anal. calcd. for $\mathrm{C}_{23} \mathrm{H}_{38} \mathrm{O}_{4} \mathrm{Si}$ : C, 67.94; H, 9,42; found: C, 68, 18; H, 9.63. ${ }^{1} \mathrm{H}-\mathrm{NMR}$ (400 $\left.\mathrm{MHz} ; \mathrm{CDCl}_{3}\right): \delta[\mathrm{ppm}]=7.23(\mathrm{dt}, J=8.9,2.5 \mathrm{~Hz}, 2 \mathrm{H}), 6.86(\mathrm{dt}, J=8,9,2.5 \mathrm{~Hz}, 2 \mathrm{H}), 4.45(\mathrm{~d}$, $\mathrm{J}=11.5,1 \mathrm{H}), 4.41(\mathrm{~d}, \mathrm{~J}=11.5,1 \mathrm{H}), 4.36(\mathrm{dd}, \mathrm{J}=6.1,1.8 \mathrm{~Hz}, 1 \mathrm{H}), 3.78$ (s, 3H), 3.66 (dd, J=9.3, $5.5,1 \mathrm{H}), 3.62(\mathrm{dd}, \mathrm{J}=9.3,5.0,1 \mathrm{H}), 3.42(\mathrm{dd}, \mathrm{J}=6.8,2.5 \mathrm{~Hz}, 1 \mathrm{H}), 3.39(\mathrm{dd}, \mathrm{J}=6.9,2.5 \mathrm{~Hz}, 1 \mathrm{H})$, 2.58 (qddd, J=6.9, 6.9,6.8, 1.8Hz, 1H), 2.01 (qddd, J=6.9, 6.2, 5.6, 5.0Hz, 1H), 1.14 (d, $\mathrm{J}=6.9 \mathrm{~Hz}, 3 \mathrm{H}), 1.00$ (d, J=6.9Hz, 3H), 0.88 (s, 9H), 0.04 (s, 6H). ${ }^{13} \mathrm{C}-\mathrm{NMR}\left(63 \mathrm{MHz}, \mathrm{CDCl}_{3}\right)$ : $\delta[\mathrm{ppm}]=159.2 ; 130.0 ; 129.2 ; 113.8 ; 87.9 ; 80.7 ; 73.4 ; 73.0 ; 67.1 ; 66.5 ; 55.2 ; 39.5 ; 29.0$; 25.9; 18.3; 17.4; 13.2; -5.3; -5.4. MS (FD, 7kV, 3mA) m/z = 406 (38), 307 (33), 121 (100). $[\alpha]_{\mathrm{D}}^{20}=-1,9^{\circ}\left(\mathrm{c}=2.0 ; \mathrm{CHCl}_{3}\right)$. IR $($ Film $) 1 / \lambda\left[\mathrm{cm}^{-1}\right]=3436 ; 2955 ; 2930 ; 2886 ; 2857 ; 1613$; $1513 ; 1463 ; 1249 ; 1090 ; 1035 ; 836 ; 776$.

(2R, 3R, 6R) 7-tert-Butyldimethylsilyloxy-1-(p-methoxy)-benzyloxy -2,6-dimethyl-hept4-yn-3-ol (20-epimer)

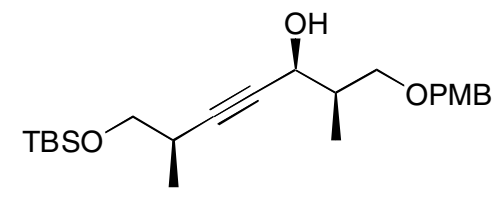

${ }^{1} \mathbf{H}-\mathbf{N M R}\left(400 \mathrm{MHz} ; \mathrm{CDCl}_{3}\right): \delta[\mathrm{ppm}]=7.23(\mathrm{dt}, J=8.9,2.5 \mathrm{~Hz}, 2 \mathrm{H}), 6.85(\mathrm{dt}, J=8,9,2.5 \mathrm{~Hz}$, 2H), $4.46(\mathrm{~d}, \mathrm{~J}=11 . \mathrm{Hz} 5,1 \mathrm{H}), 4.42(\mathrm{~d}, \mathrm{~J}=11.5 \mathrm{~Hz}, 1 \mathrm{H}), 4.37(\mathrm{ddd}, \mathrm{J}=8.0,3.2,1.5 \mathrm{~Hz}, 1 \mathrm{H})$, $3.79(\mathrm{~s}, 3 \mathrm{H}), 3.64-3.70(\mathrm{~m}, 2 \mathrm{H}), 3.42-3.49(\mathrm{~m}, 3 \mathrm{H}), 2.60(\mathrm{qtd}, \mathrm{J}=6.9,6.8,1.5 \mathrm{~Hz}, 1 \mathrm{H}), 2.21$ $(\mathrm{m}, 1 \mathrm{H}), 1.16$ (d, J = 7.0 Hz, 3H), 0.89 (d, J = 7.0 Hz, 3H), 0.89 (s, 9H), 0.04 (s, 6H). ${ }^{13} \mathrm{C}-$ NMR (100.6 MHz; $\left.\mathrm{CDCl}_{3}\right): \delta[\mathrm{ppm}]=159.3,129.9,129.3,113.8,88.0,80.0,73.3,73.1$, $67.2,66.8,55.2,38.7,29.1,25.9 ; 18.3 ; 17.4,12.8,-5.3,-5.4 .[\alpha]_{\mathrm{D}}^{20}=-19.8^{\circ}(\mathrm{c}=2.0 ;$ $\left.\mathrm{CHCl}_{3}\right)$. 
$(2 R, 3 S, 6 R)$ 7-tert-Butyldimethylsilyloxy-1-p-methoxy-benzyloxy -2,6-dimethyl-heptan3-ol (21)

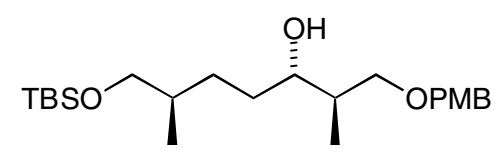

20 or $27(1.5 \mathrm{~g}, 3.7 \mathrm{mmol})$ in methanol $(50 \mathrm{~mL})$ was stirred with hydrazine hydrate $(20 \mathrm{~mL})$ and $\mathrm{Cu}(\mathrm{OAc})_{2}(20 \mathrm{mg})$ under dioxygen for $12 \mathrm{~h}$. Additional hydrazine hydrate $(20 \mathrm{~mL})$ and $\mathrm{Cu}(\mathrm{OAc})_{2}(20 \mathrm{mg})$ were added and stirring was continued for another $20 \mathrm{~h}$. Satd. $\mathrm{NH}_{4} \mathrm{Cl}(20$ $\mathrm{mL}$ ) was added and the mixture was concentrated under reduced pressure. The residue waas diluted with ether and worked up as usual to give after chromatography (hexane/ethyl acetate $\left.3: 1, \mathrm{R}_{\mathrm{f}}=0.37\right) 21(1.26 \mathrm{~g}, 83 \%)$ as a colorless oil. Anal. Calcd. for $\mathrm{C}_{23} \mathrm{H}_{42} \mathrm{O}_{4} \mathrm{Si}: \mathrm{C}, 67.27$; $\mathrm{H}, 10.31$; found: C, 67.52; H, 10,38. ${ }^{1} \mathrm{H}-\mathrm{NMR}\left(400 \mathrm{MHz} ; \mathrm{CDCl}_{3}\right): \delta[\mathrm{ppm}]=7.24(\mathrm{~d}$, $J=8.7 \mathrm{~Hz}, 2 \mathrm{H}), 6.87(\mathrm{~d}, J=8,7 \mathrm{~Hz} 2 \mathrm{H}), 4.44$ (s, 2H), 3.80 (s, 3H), 3.57 (dd, J= 9.2, 4.2Hz, 1H), 3.48 (dd, J=9.8, 6.4Hz, 1H), 3.45 (m, 1H), 3.43 (dd, 9.2, 7.3Hz, 1H), 3.36 (dd, J=9.8, 6.4Hz, $1 \mathrm{H}), 3.31(\mathrm{~d}, \mathrm{~J}=3.7 \mathrm{~Hz}, 1 \mathrm{H}), 1.83(\mathrm{~m}, 1 \mathrm{H}), 1.58(\mathrm{~m}, 3 \mathrm{H}), 1.37(\mathrm{~m}, 1 \mathrm{H}), 1.14(\mathrm{~m}, 1 \mathrm{H}), 0.89(\mathrm{~d}$, $\mathrm{J}=10.3 \mathrm{~Hz}, 3 \mathrm{H}), 0.89$ (s, 9H), 0.88 (d, J=7.8Hz, 3H), 0.03 (s, 6H). ${ }^{13} \mathrm{C}-\mathrm{NMR}(100.3 \mathrm{MHz}$; $\left.\mathrm{CDCl}_{3}\right): \delta[\mathrm{ppm}]=159.3,129.9,129.3,113.8,76.5,74.8,73.1,68.2,55.2,38.0,36.0,32.3$, 28.7, 26.0, 18.3, 17.0, 14.1, -5.4. MS (EI, 70eV, 200 $\mathrm{C}): \mathrm{m} / \mathrm{z}=411[\mathrm{M}+1],(7), 278$ (13), 242 (14), 236 (16), $199(28), 173(31), 145(33), 121(100) .[\alpha]_{\mathrm{D}}^{20}=-12.8^{\circ}(\mathrm{c}=1,95$;

$\mathrm{CHCl}_{3}$ ). IR (Film) $1 / \lambda\left[\mathrm{cm}^{-1}\right]=3489,2954,2857,1613,1514,1463,1361,1302,1250,1173$, $1091,1037,836,776$.

$(2 R, 3 R, 6 R)$ 7-tert-Butyldimethylsilyloxy-1-p-methoxybenzyloxy -2,6-dimethyl-heptan3-ol (21a)

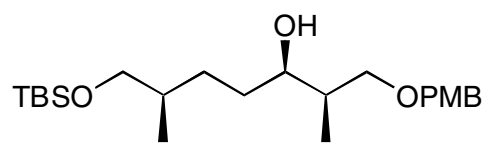

Obtained from 28 analogously. Anal. Calcd. for $\mathrm{C}_{23} \mathrm{H}_{42} \mathrm{O}_{4} \mathrm{Si}$ : C, 67.27; H, 10.31; found:

C, 67.51; H, 10.32. ${ }^{1} \mathrm{H}-\mathrm{NMR}\left(400 \mathrm{MHz} ; \mathrm{CDCl}_{3}\right): \delta[\mathrm{ppm}]=7.24(\mathrm{~d}, J=8.7 \mathrm{~Hz}, 2 \mathrm{H}), 6.87$

$(\mathrm{d}, J=8,7 \mathrm{~Hz}, 2 \mathrm{H}), 4.46(\mathrm{~d}, \mathrm{~J}=11.6 \mathrm{~Hz}, 1 \mathrm{H}), 4.42(\mathrm{~d}, \mathrm{~J}=11.6 \mathrm{~Hz}, 1 \mathrm{H}), 3.80(\mathrm{~s}, 3 \mathrm{H}), 3.71(\mathrm{~m}$, $1 \mathrm{H}), 3.49(\mathrm{~d}, \mathrm{~J}=5.5 \mathrm{~Hz}, 2 \mathrm{H}), 3.45(\mathrm{dd}, \mathrm{J}=9.8,5.9 \mathrm{~Hz}, 1 \mathrm{H}), 3.49(\mathrm{dd}, \mathrm{J}=9.8,6.3 \mathrm{~Hz}, 1 \mathrm{H})$, 
$2.59(\mathrm{~m}, 1 \mathrm{H}), 1.86(\mathrm{~m}, 1 \mathrm{H}), 1.59(\mathrm{~m}, 1 \mathrm{H}), 1.20-1.52(\mathrm{~m}, 4 \mathrm{H}), 0.91(\mathrm{~d}, \mathrm{~J}=7.1 \mathrm{~Hz}, 3 \mathrm{H}), 0.89$ $(\mathrm{s}, 9 \mathrm{H}), 0.88(\mathrm{~d}, \mathrm{~J}=6.7 \mathrm{~Hz}, 3 \mathrm{H}), 0.03(\mathrm{~s}, 6 \mathrm{H}) .{ }^{13} \mathrm{C}-\mathrm{NMR}\left(100.3 \mathrm{MHz} ; \mathrm{CDCl}_{3}\right): \delta[\mathrm{ppm}]=$ 159.2, 130.2, 129.2, 113.8, 74.4, 74.3, 73.0, 68.3, 55.2, 38.0, 35.8, 31.4, 29.8, 25.9, 18.3, 16.8, 10.7, -5.4. $[\alpha]_{\mathrm{D}}^{20}=+20.5^{\circ}\left(\mathrm{c}=2.0 ; \mathrm{CHCl}_{3}\right)$.

$(2 R, 3 S, 6 R)$ 7-tert-Butyldimethylsilyloxy-1,3-p-methoxybenzyliden-dioxy-2,6-dimethylheptane (22)

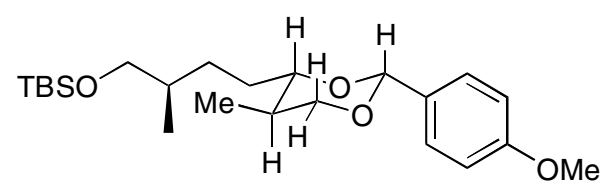

Molecular sieves $4 \AA$ ( $3 \mathrm{~g}$ ) were finely ground and suspended in dichloromethane (15 mL). 21 $(0.57 \mathrm{~g}, 1.4 \mathrm{mmol})$ in dichloromethane $(15 \mathrm{~mL})$ was added and the mixture was cooled to $0^{\circ} \mathrm{C}$. DDQ $(0.35 \mathrm{~g}, 1.55 \mathrm{mmol})$ in THF $(3 \mathrm{~mL})$ was added slowly. The mixture was stirred for $2 \mathrm{~h}$ and filtered over celite and concentrated under reduced pressure. The residue was chromatographed (hexanes/ethyl acetate $\left.3: 1, \mathrm{R}_{\mathrm{f}}=0.61\right)$ to give $22(0.43 \mathrm{~g}, 76 \%)$ as a colorless oil. Anal. Calcd. for $\mathrm{C}_{23} \mathrm{H}_{40} \mathrm{O}_{4} \mathrm{Si}$ : C, 67,60; H, 9,87; found: C, 67.85; H, 9.76. ${ }^{1} \mathrm{H}-\mathrm{NMR}\left(400 \mathrm{MHz} ; \mathrm{CDCl}_{3}\right): \delta[\mathrm{ppm}]=7.42(\mathrm{dt}, J=8.8,2.0 \mathrm{~Hz}, 2 \mathrm{H}), 6.88(\mathrm{dt}, J=8,8$, $2.0 \mathrm{~Hz} 2 \mathrm{H}), 5.44(\mathrm{~s}, 1 \mathrm{H}), 4.08(\mathrm{dd}, \mathrm{J}=11.2,4.8 \mathrm{~Hz}, 1 \mathrm{H}), 3.80(\mathrm{~s}, 3 \mathrm{H}), 3.48(\mathrm{dd}, \mathrm{J}=11.2$, $11.1 \mathrm{~Hz}, 1 \mathrm{H}), 3.48(\mathrm{dd}, \mathrm{J}=9.8,6.2 \mathrm{~Hz}, 1 \mathrm{H}), 3.39(\mathrm{dd}, \mathrm{J}=9.8,6.4 \mathrm{~Hz}, 1 \mathrm{H}), 3.38(\mathrm{ddd}, 9.8,8.0$, 2.5Hz, 1H), $1.86(\mathrm{ddqd}, \mathrm{J}=11.1,9.9,6.7,4.8 \mathrm{~Hz}, 1 \mathrm{H}), 1.81-1.70(\mathrm{~m}, 2 \mathrm{H}), 1.61(\mathrm{~m}, 1 \mathrm{H}), 1.50$ (m, 1H), $1.17(\mathrm{~m}, 1 \mathrm{H}), 0.90$ (d, J = 7.0Hz, 3H), 0.89 (s, 9H), 0.79 (d, J=6.7Hz, 3H), 0.04 (s, $6 \mathrm{H}) .{ }^{13} \mathrm{C}-\mathrm{NMR}\left(63 \mathrm{MHz} ; \mathrm{CDCl}_{3}\right): \delta[\mathrm{ppm}]=159.8,131.5,127.3,113.5,100.9 ; 83.5,73.0$, $68.1,55.3,35.9,33.8,30.2,28.6,25.9,18.3,16.9,12.5,-5.4$.

MS (EI, 70eV, 200 $\mathrm{C}): \mathrm{m} / \mathrm{z}=409$ [M+1] (9), 408 [M] (19), 351 [M-57] (54), 173(14), 135 (36), 121 (100). Drehwert: $[\alpha]_{\mathrm{D}}^{20}=-18.0^{\circ}\left(\mathrm{c}=2,1 ; \mathrm{CHCl}_{3}\right) . \mathrm{IR}\left(\right.$ Film) $1 / \lambda\left[\mathrm{cm}^{-1}\right]=2955$, 2930, 2856, 1616, 1518, 1462, 1391, 1362, 1302, 1250, 1171, 1117, 1089, 1038, 1011, 836, 776.

$(2 R, 3 R, 6 R)$ 7-tert-Butyldimethylsilyloxy-1,3-p-methoxybenzyliden-dioxy-2,6-dimethylheptane (30)

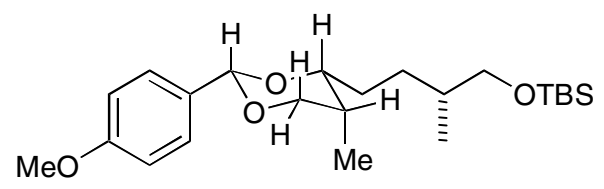


Obtained from 21a analogously. Anal. Calcd. for $\mathrm{C}_{23} \mathrm{H}_{40} \mathrm{O}_{4} \mathrm{Si}: \mathrm{C}, \quad 67.60 ; \mathrm{H}, 9.87$; found: $\mathrm{C}$, 67.85; H, 9.76. ${ }^{1} \mathrm{H}-\mathrm{NMR}\left(400 \mathrm{MHz} ; \mathrm{CDCl}_{3}\right): \delta[\mathrm{ppm}]=7.43(\mathrm{dt}, J=8.7,2.2 \mathrm{~Hz}, 2 \mathrm{H}), 6.89$ (dt, $J=8.7,2.2 \mathrm{~Hz} 2 \mathrm{H}), 5.45(\mathrm{~s}, 1 \mathrm{H}), 4.07(\mathrm{dd}, \mathrm{J}=11.1,2.4 \mathrm{~Hz}, 1 \mathrm{H}), 4.01$ (dd, J = 11.1, $0.9 \mathrm{~Hz}, 1 \mathrm{H}), 3.86(\mathrm{ddd}, 7.5,6.0,2.2 \mathrm{~Hz}, 1 \mathrm{H}), 3.80(\mathrm{~s}, 3 \mathrm{H}), 3.45(\mathrm{dd}, \mathrm{J}=9.8,6.0 \mathrm{~Hz}, 1 \mathrm{H}), 3.42$ $(\mathrm{dd}, \mathrm{J}=9.8,6.2 \mathrm{~Hz}, 1 \mathrm{H}), 1.64-1.71(\mathrm{~m}, 1 \mathrm{H}), 1.57-1.63(\mathrm{~m}, 1 \mathrm{H}), 1.55$ (qddd, $\mathrm{J}=7.0,2.4,2.2$, $0.9 \mathrm{~Hz}, 1 \mathrm{H}), 1.38-1.46(\mathrm{~m}, 2 \mathrm{H}), 1.19(\mathrm{~m}, 1 \mathrm{H}), 1.18(\mathrm{~d}, \mathrm{~J}=7.0 \mathrm{~Hz}, 3 \mathrm{H}), 0.90(\mathrm{~s}, 9 \mathrm{H}), 0.89$ (d, $\mathrm{J}=7.0 \mathrm{~Hz}, 3 \mathrm{H}), 0.04(\mathrm{~s}, 6 \mathrm{H}) .{ }^{13} \mathrm{C}-\mathrm{NMR}\left(100.6 \mathrm{MHz} \mathrm{CDCl}_{3}\right): \delta[\mathrm{ppm}]=159.9,131.7,127.4$, $113.6,101.7 ; 80.1,73.9,68.0,55.3,35.8,31.6,30.2,28.5,25.9,18.3,16.8,11.0$, 5.4. $[\alpha]_{\mathrm{D}}^{20}=+13.2^{\circ}\left(\mathrm{c}=2.0 ; \mathrm{CHCl}_{3}\right)$.

(2R, 3S, 6R) 7-tert-Butyldimethylsilyloxy-3-p-methoxybenzyloxy-2,6-dimethyl-heptan-1ol (23)

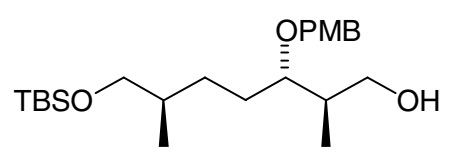

22 (2.6 g, $6.4 \mathrm{mmol})$ in $\mathrm{CH}_{2} \mathrm{Cl}_{2}(30 \mathrm{~mL})$ was treated dropwise at $-40^{\circ} \mathrm{C}$ with DiBAL-H $(1.2$ $\mathrm{M}$ in toluene, $5.3 \mathrm{~mL}, 6.4 \mathrm{mmol})$ and stirred for $2 \mathrm{~h}$ at $-10^{\circ} \mathrm{C}$. Satd. aqu. tartaric acid $(20 \mathrm{~mL})$ and ether $(30 \mathrm{~mL})$ were added and the mixture was stirred at $0^{\circ} \mathrm{C}$, until a clean two-phase system was formed. The organic layer was separated and worked up as usual to give after chromatography (hexanes/ethyl acetate $\left.3: 1 ; \mathrm{R}_{\mathrm{f}}=0.31\right) \mathbf{2 3}(2.15 \mathrm{~g}, 82 \%)$ as a colorless oil. Anal. calcd. for $\mathrm{C}_{23} \mathrm{H}_{42} \mathrm{O}_{4} \mathrm{Si}$ : C, 67.27; H, 10.31; found: C, 67.10; H, 10.62. ${ }^{1} \mathrm{H}-\mathrm{NMR}(400$ $\left.\mathrm{MHz} ; \mathrm{CDCl}_{3}\right): \delta[\mathrm{ppm}]=7.26(\mathrm{dt}, J=8.6,2.5 \mathrm{~Hz}, 2 \mathrm{H}), 6.87(\mathrm{dt}, J=8.6,2.5 \mathrm{~Hz} 2 \mathrm{H}), 4.55(\mathrm{~d}$, $\mathrm{J}=11.0 \mathrm{~Hz}, 1 \mathrm{H}), 4.37(\mathrm{~d}, \mathrm{~J}=11.0 \mathrm{~Hz}, 1 \mathrm{H}), 3.80(\mathrm{~s}, 3 \mathrm{H}), 3.66(\mathrm{ddd}, \mathrm{J}=10.5,6.0,4.5 \mathrm{~Hz}, 1 \mathrm{H})$, $3.55(\mathrm{ddd}, \mathrm{J}=10.5,6.0,4.5 \mathrm{~Hz}, 1 \mathrm{H}), 3.45(\mathrm{dd}, \mathrm{J}=9.5,6.0 \mathrm{~Hz}, 1 \mathrm{H}), 3.41(\mathrm{dd}, \mathrm{J}=9.5,6.0 \mathrm{~Hz}$, $1 \mathrm{H}), 3.36-3.42(\mathrm{~m}, 1 \mathrm{H}), 2.85(\mathrm{t}, \mathrm{J}=4.5 \mathrm{~Hz}, 1 \mathrm{H}), 1.89(\mathrm{~m}, 1 \mathrm{H}), 1.71(\mathrm{~m}, 1 \mathrm{H}), 1.47-1.62(\mathrm{~m}$, 3H), $1.15(\mathrm{~m}, 1 \mathrm{H}), 0.91$ (d, J = 7.0Hz, 3H), 0.90 (s, 9H), 0.89 (d, J=6.0Hz, 3H), 0.04 (s, 6H). ${ }^{13} \mathrm{C}-\mathrm{NMR}\left(100.6 \mathrm{MHz} ; \mathrm{CDCl}_{3}\right): \delta[\mathrm{ppm}]=159.3,130.4,129.4,113.9,84.1,71.3,68.1,66.9$, $55.3,37.7,36.0,28.0,27.6,25.9,18.3,16.8,14.2,-5.3 . \mathrm{MS}\left(\mathrm{EI}, 70 \mathrm{eV}, 60^{\circ} \mathrm{C}\right) . \mathrm{m} / \mathrm{e}=410$ [M] (5), 353 [M-57] (8), 274 (13), 217 (9), 173 (10), 163 (10), 137 (57), 121 (100); HRMS calcd. for : $\mathrm{C}_{23} \mathrm{H}_{42} \mathrm{O}_{4} \mathrm{Si}: 410.2852$; found : 410.2870. $[\alpha]_{\mathrm{D}}^{20}=+29.2^{\circ}\left(\mathrm{c}=2 ; \mathrm{CHCl}_{3}\right)$. IR 
(Film) $1 / \lambda\left[\mathrm{cm}^{-1}\right]=3436,2955,2930,2857,1613,1514,1464,1387,1361,1302,1250$, 1173, 1090, 1038, 1007, 939, 836, 776.

$(2 R, 3 S, 6 R)$ 7-tert-Butyldimethylsilyloxy-3-p-methoxybenzyloxy-2,6-dimethyl-heptan-1al (24)

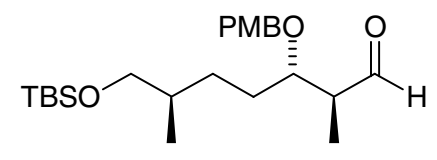

23 (2.4 g, 9.4 mmol) was oxidized under Swern conditions (TP 1) to give after chromatography (hexanes/ ethyl acetate $\left.3: 1 ; \mathrm{R}_{\mathrm{f}}=0.58\right) \mathbf{2 4}(2.3 \mathrm{~g}, 97 \%)$ as a colorless oil. Anal calcd. for $\mathrm{C}_{23} \mathrm{H}_{40} \mathrm{O}_{4} \mathrm{Si}$ : C, 67.60; H, 9.87; found: C, 67.70; H, 10.02.

${ }^{1} \mathrm{H}-\mathrm{NMR}\left(400 \mathrm{MHz} ; \mathrm{CDCl}_{3}\right): \delta[\mathrm{ppm}]=9.73(\mathrm{~d}, \mathrm{~J}=2.2 \mathrm{~Hz}, 1 \mathrm{H}), 7.24(\mathrm{dt}, J=8.5,2.5 \mathrm{~Hz}, 2 \mathrm{H})$, $6.87(\mathrm{dt}, J=8,5,2.5 \mathrm{~Hz}, 2 \mathrm{H}), 4.51(\mathrm{~d}, \mathrm{~J}=11.0 \mathrm{~Hz}, 1 \mathrm{H}), 4.41(\mathrm{~d}, \mathrm{~J}=11.0 \mathrm{~Hz}, 1 \mathrm{H}), 3.80(\mathrm{~s}, 3 \mathrm{H})$, $3.67(\mathrm{q}, \mathrm{J}=5.8 \mathrm{~Hz}, 1 \mathrm{H}), 3.43(\mathrm{dd}, \mathrm{J}=10.0,5.8 \mathrm{~Hz}, 1 \mathrm{H}), 3.40(\mathrm{dd}, \mathrm{J}=10.0,5.8 \mathrm{~Hz}, 1 \mathrm{H}), 2.66$ (qdd, J = 7.0, 6.9, 2.2Hz, 1H), 1.50-1.64 (m, 4H), 1.10-1.18 (m, 1H), 1.07 (d, J = 7.0Hz, 3H), 0.89 (s, 9H), $0.88(\mathrm{~d}, \mathrm{~J}=6.5 \mathrm{~Hz}, 3 \mathrm{H}), 0.04(\mathrm{~s}, 6 \mathrm{H})$.

${ }^{13} \mathrm{C}-\mathrm{NMR}\left(100,6 \mathrm{MHz} ; \mathrm{CDCl}_{3}\right): \delta[\mathrm{ppm}]=204.6,159.2,130.3$ 129.3, 113.8, 79.4, 71.2, 68.0, 55.3, 49.3, 35.9, 28.3, 28.1, 25.9, 18.3, 16.8, 10.0, -5.4. MS: (EI, $\left.70 \mathrm{eV}, 80^{\circ} \mathrm{C}\right) \mathrm{m} / \mathrm{z}=$ 351 [M-57] (8), 325(5), 282 (4), 255 (5), 223 (8), 213 (34), 173 (67), 138 (25), 121 (100); HRMS calcd for : $\mathrm{C}_{19} \mathrm{H}_{31} \mathrm{O}_{4} \mathrm{Si}: 315.1992\left[\mathrm{M}-\mathrm{C}_{4} \mathrm{H}_{9}\right]$; found: 315.1981 . $[\alpha]_{\mathrm{D}}^{20}=+29.8^{\circ}(\mathrm{c}=$ 2.0; $\mathrm{CHCl}_{3}$ ). IR (Film) 1/ $\lambda\left[\mathrm{cm}^{-1}\right]$ 2955, 2931, 2857, 1727, 1614, 1514, 1463, 1389,1302, 1249, 1173, 1090, 1037, 1007, 939, 837, 776.

$(2 R / S, 3 S, 4 S, 7 R)-8$ - tert-Butyldimethylsilyloxy -4-p-methoxy-benzyloxy-3,7-dimethyl-2octanol

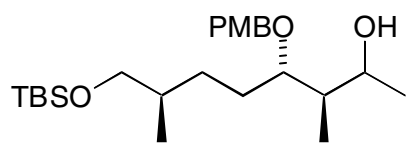

$24(1.2 \mathrm{~g}, 2.9 \mathrm{mmol})$ in $\mathrm{Et}_{2} \mathrm{O}(20 \mathrm{~mL})$ was treated dropwise at $-10^{\circ} \mathrm{C}$ with $3 \mathrm{M} \mathrm{MeMgBr}$ in $\mathrm{Et}_{2} \mathrm{O}(3 \mathrm{~mL}, 9 \mathrm{mmol})$. The mixture was stirred at $0^{\circ} \mathrm{C}$ for $1 \mathrm{~h}$ and then worked up as usual to give after chromatography (hexanes/ethyl acetate $\left.3: 1, \mathrm{R}_{\mathrm{f}}=0.46\right)(2 R / S, 3 S, 4 S, 7 R)$-8- tertButyldimethylsilyloxy -4-p-methoxybenzyloxy-3,7-dimethyl-2-octanol (1.15 g, $92 \%)$ as a 
colorless diastereomeric mixture. ${ }^{1} \mathrm{H}-\mathrm{NMR}\left(250 \mathrm{MHz} ; \mathrm{CDCl}_{3}\right): \delta[\mathrm{ppm}]=7.26(\mathrm{dt}, J=8.7$, 2.4Hz, 2H), 6.87 (dt, J=8,1,2.4Hz, 2H), 4.57 (d, J=10.9Hz, 0.5H), 4.53 (d, J=10.9Hz, 0.5H), $4.38(\mathrm{~d}, \mathrm{~J}=10.9 \mathrm{~Hz}, 0.5 \mathrm{H}), 4.36(\mathrm{~d}, \mathrm{~J}=10.9 \mathrm{~Hz}, 0.5 \mathrm{H}), 4.08(\mathrm{~m}, 0.5 \mathrm{H}), 3.80(\mathrm{~s}, 3 \mathrm{H}), 3.73(\mathrm{~m}$, $0.5 \mathrm{H}), 3.43(\mathrm{~m}, 3 \mathrm{H}), 3.52(\mathrm{dd}, \mathrm{J}=6.8,2.0 \mathrm{~Hz}, 1 \mathrm{H}), 1.42-1-74(\mathrm{~m}, 5 \mathrm{H}), 1.15(\mathrm{~m}, 1 \mathrm{H}), 1.14(\mathrm{~d}$, $\mathrm{J}=6.1 \mathrm{~Hz}, 1.5 \mathrm{H}), 1.12(\mathrm{~d}, \mathrm{~J}=6.5 \mathrm{~Hz}, 1.5 \mathrm{H}), 0.94(\mathrm{~d}, \mathrm{~J}=7.1 \mathrm{~Hz}, 1.5 \mathrm{H}), 0.90(\mathrm{~d}, \mathrm{~J}=6.6 \mathrm{~Hz}, 3 \mathrm{H})$, $0.90(\mathrm{~s}, 9 \mathrm{H}), 0.79(\mathrm{~d}, \mathrm{~J}=7.0 \mathrm{~Hz}, 1.5 \mathrm{H}), 0.04(\mathrm{~s}, 6 \mathrm{H}) .{ }^{13} \mathrm{C}-\mathrm{NMR}\left(63 \mathrm{MHz} ; \mathrm{CDCl}_{3}\right): \delta[\mathrm{ppm}]=$ $159.2 ; 130.3 ; 130.2 ; 129.5 ; 129.4 ; 113.8 ; 83.7 ; 83.5 ; 71.7 ; 71.4 ; 70.9 ; 68.1 ; 68.0 ; 67.3 ; 55.2$; $42.7 ; 40.3 ; 36.0 ; 35.9 ; 28.2 ; 27.5 ; 27.0 ; 25.9 ; 21.0 ; 20.0 ; 18.3 ; 17.0 ; 16.7 ; 12.8 ; 11.6 ;-5.2$. MS: (EI, $70 \mathrm{eV}, 80^{\circ} \mathrm{C}$ ) m/z = 404 [M-15] (3), 365 [M-57] (9), 227 (11), 174 (12), 173 (58), 155 (12), 145 (12), 121 (100). HRMS calcd. for $\mathrm{C}_{24} \mathrm{H}_{44} \mathrm{O}_{4} \mathrm{Si}$ : 424.3001 ; found: 424.3024. $[\alpha]_{\mathrm{D}}^{20}=+24.8^{\circ}\left(\mathrm{c}=2.0 ; \mathrm{CHCl}_{3}\right) . \mathrm{IR}($ Film $) 1 / \lambda\left[\mathrm{cm}^{-1}\right]=3469,2956,2930,2896,2857$, 1614, 1515, 1463, 1387, 1361,1302, 1250, 1173, 1091, 1039, 1007, 939, 836, 776.

$(3 R, 4 S, 7 R)$ 8-tert-Butyldimethylsilyloxy-4-p-methoxybenzyloxy-3,7-dimethyl-octan-2on (5)

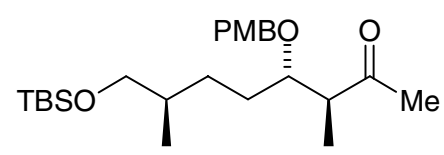

$(2 R / S, 3 S, 4 S, 7 R)-8$ - tert-Butyldimethylsilyloxy -4- $p$-methoxybenzyloxy-3,7-dimethyl-2octanol (0.89 g, $2.1 \mathrm{mmol})$ was oxidized under Swern conditions (TP 1) to give after chromatography (hexanes/ ethyl acetate $\left.3: 1 ; \mathrm{R}_{\mathrm{f}}=0.56\right)$ ketone $5(0.81 \mathrm{~g}, 92 \%)$ as a colorless oil. Anal. Calcd. for $\mathrm{C}_{24} \mathrm{H}_{42} \mathrm{O}_{4}$ : C, 68.20; H, 10.02; found: C, 68.41; H, 9.95. NMR $\left(250 \mathrm{MHz} ; \mathrm{CDCl}_{3}\right): \delta[\mathrm{ppm}]=7.21(\mathrm{dt}, J=8.5,2.2 \mathrm{~Hz}, 2 \mathrm{H}), 6.86(\mathrm{dt}, J=8,5,2.2 \mathrm{~Hz}, 2 \mathrm{H}), 4.45$ $(\mathrm{d}, \mathrm{J}=11.0 \mathrm{~Hz}, 1 \mathrm{H}), 4.34$ (d, J=11.0Hz, 1H), 3.80 (s, 3H), 3.64 (ddd, J=7.9, 5.5, 3.6Hz, 1H ), $3.44(\mathrm{dd}, \mathrm{J}=9.9,5.8 \mathrm{~Hz}, 1 \mathrm{H}), 3.39$ (dd, J=9.9, 5.8Hz, 1H), 2.84(qd, J=7.3, 6.9Hz, 1H), 2.15 (s, 3H), 1.40-168 (m, 4H), 1.10-1.25 (m, 1H), 1.02 (d, J=6.9Hz, 3H), 0.89 (s, 9H), 0.88 (d, $\mathrm{J}=6.5 \mathrm{~Hz}, 3 \mathrm{H}), 0.04(\mathrm{~s}, 6 \mathrm{H}) .{ }^{13} \mathrm{C}-\mathrm{NMR}\left(63 \mathrm{MHz} ; \mathrm{CDCl}_{3}\right): \delta[\mathrm{ppm}]=212.0 ; 159.0 ; 130.4$; $129.3 ; 113.6 ; 80.6 ; 71.4 ; 67.9 ; 55.1 ; 49.8 ; 35.8 ; 30.2 ; 27.7 ; 27.5 ; 25.8 ; 18.2 ; 16.8 ; 12.37$; 5.4. MS: (Fd, $7 \mathrm{kV}, 3 \mathrm{~mA}) \mathrm{M}=424$ [M] (100); (EI, $\left.70 \mathrm{eV}, 80^{\circ} \mathrm{C}\right) \mathrm{m} / \mathrm{z}=404$ [M-14] (3), 365 [M-57] (9), 227 (11), 174 (12), 173 (58), 155 (12), 145 (12), 121 (100). HRMS calcd. for : $\mathrm{C}_{20} \mathrm{H}_{33} \mathrm{O}_{4} \mathrm{Si}: 365.2148$; found: 365.2160 . $[\alpha]_{\mathrm{D}}^{20}=+30.7^{\circ}(\mathrm{c}=1.0$; $\left.\mathrm{CHCl}_{3}\right)$. 
IR (Film) $1 / \lambda\left[\mathrm{cm}^{-1}\right]=2955,2857,1717,1612,1514,1460,1359,1250,1174,1090,1037$, 837, 776.

Dimethyl-(3R) 3-methyl-4-p-methoxybenzyloxy-2-oxo-butane-1-phosphonate (25)

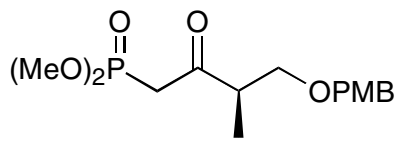

LDA was prepared from $125 \mathrm{~mL}(200 \mathrm{mmol}) 1.6 \mathrm{M}$ n-butyl-lithium in hexane and $28 \mathrm{~mL}$ diisopropylamine in THF $(100 \mathrm{~mL})$ at $-20^{\circ} \mathrm{C}$. After $1 \mathrm{~h}$ at $0^{\circ} \mathrm{C}$ the LDA solution was cooled to $-78^{\circ} \mathrm{C}$ and dimethyl methanephosphonate $(24.8 \mathrm{~g}, 200 \mathrm{mmol})$ in THF $(100 \mathrm{~mL})$ was added slowly. After $30 \mathrm{~min} \mathbf{1 8}(23.8 \mathrm{~g}, 100 \mathrm{mmol})$ was added in THF $(50 \mathrm{~mL})$ at $-78^{\circ} \mathrm{C}$ over $1 \mathrm{~h}$. After additional 30 min ther mixture was quenched with satd. $\mathrm{NH}_{4} \mathrm{Cl}(150 \mathrm{~mL})$ Workup with ether furnished after chromatography (hexanes / ethyl acetate/ methanol 4: 4: 1, $\left.\mathrm{R}_{\mathrm{f}}=0.20\right) 25$ (33 g, $91 \%$ ) as a colorless oil. Anal.calcd. for $\mathrm{C}_{15} \mathrm{H}_{23} \mathrm{O}_{6} \mathrm{P}: \mathrm{C}, 54.54 ; \mathrm{H}, 7.02$; found: C, 54.27; H, 7.24. ${ }^{1} \mathrm{H}-\mathrm{NMR}\left(250 \mathrm{MHz} ; \mathrm{CDCl}_{3}\right): \delta[\mathrm{ppm}]=7.23(\mathrm{dt}, J=8.7,2.5 \mathrm{~Hz}, 2 \mathrm{H})$, $6.87(\mathrm{dt}, J=8,7,2.5 \mathrm{~Hz}, 2 \mathrm{H}), 4.44(\mathrm{~s}, 2 \mathrm{H}), 3.79(\mathrm{~s}, 3 \mathrm{H}), 3.68(\mathrm{~s}, 3 \mathrm{H}), 3.63(\mathrm{dd}, \mathrm{J}=9.2$, $7.3 \mathrm{~Hz}, 1 \mathrm{H}), 3.45(\mathrm{dd}, \mathrm{J}=9.2,5.9,1 \mathrm{H}), 2.76(\mathrm{dqd}, \mathrm{J}=7.3,7.1,5.9 \mathrm{~Hz}, 1 \mathrm{H}), 1.16(\mathrm{~d}, \mathrm{~J}=$ 7.1Hz, 3H). ${ }^{13} \mathrm{C}-\mathrm{NMR}\left(63 \mathrm{MHz} ; \mathrm{CDCl}_{3}\right): \delta[\mathrm{ppm}]=175.3,159.1,130.2$ 129.2, 113.7, 72.7, 71.6, 55.2, 51.7, 40.1, 14.0. MS (EI, $\left.70 \mathrm{eV}, 100^{\circ} \mathrm{C}\right): \mathrm{m} / \mathrm{z}=238$ [M] (18), 151(7), 137 (88),

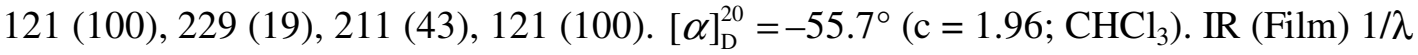
$\left[\mathrm{cm}^{-1}\right]=3469,2956,2855,1715,1674,1613,1586,1514,1463,1421,1364,1302,1252$, 11781, 1028, 879, 812;

(2R, 6R) 7-tert-Butyldimethylsilyloxy-1-p-methoxybenzyloxy -2,6-dimethyl-hept-4-en-3one (26)

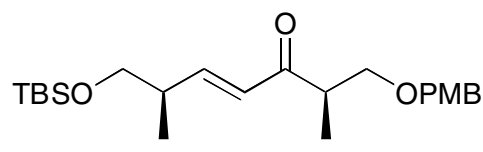

25 (11.4 g, $34.5 \mathrm{mmol})$ in THF $(70 \mathrm{~mL})$ was treated with $\mathrm{LiOH} \cdot \mathrm{H}_{2} \mathrm{O}(1.45 \mathrm{~g})$ and the mixture was stirred at $0^{\circ} \mathrm{C}$ for $15 \mathrm{~min} .18(7.0 \mathrm{~g}(34.5 \mathrm{mmol})$ in $\mathrm{THF}(40 \mathrm{~mL})$ and the mixture was 
stirred for $20 \mathrm{~h}$ at ambient temperature. Usual workup furnished after chromatography (hexanes/ethyl acetate $\left.3: 1 ; \mathrm{R}_{\mathrm{f}}=0.65\right) \mathbf{2 6}(12.4 \mathrm{~g}, 89 \%)$ as a colorless oil. Anal calcd. for $\mathrm{C}_{23} \mathrm{H}_{38} \mathrm{O}_{4} \mathrm{Si}: \mathrm{C}, 67.94 ; \mathrm{H}, 9.42$; found: C, 68.14; H, 9.53. ${ }^{1} \mathrm{H}-\mathrm{NMR}\left(400 \mathrm{MHz} ; \mathrm{CDCl}_{3}\right.$ ): $\delta$ [ppm] $=7.22(\mathrm{~d}, J=8.7 \mathrm{~Hz}, 2 \mathrm{H}), 6.86(\mathrm{~d}, J=8,7 \mathrm{~Hz}, 2 \mathrm{H}), 6.83(\mathrm{dd}, \mathrm{J}=15.9,7.3 \mathrm{~Hz}) 5.60$ (dd, $\mathrm{J}=15.6,6.9 \mathrm{~Hz}, 1 \mathrm{H}), 5.45$ (dd, 15.6, 7.2Hz, 1H), 4.44 (s, 2H), 3.94 (ddd, J=7.2, 7.2, 2.8Hz, 1H), $3.80(\mathrm{~s}, 3 \mathrm{H}), 3.54(\mathrm{~m}, 2 \mathrm{H}), 3.41(\mathrm{~m}, 2 \mathrm{H}), 3.29(\mathrm{~d}, \mathrm{~J}=2.8 \mathrm{~Hz}, 1 \mathrm{H}), 2.33(\mathrm{~m}, 1 \mathrm{H}), 1.87(\mathrm{~m}$, $1 \mathrm{H}), 0.99$ (d, J=6.8Hz, 3H), 0.89 (s, 9H), 0.84 (d, J=7.0Hz, 3H), 0.03 (s, 6H). ${ }^{13} \mathrm{C}-\mathrm{NMR}(63$ $\left.\mathrm{MHz} ; \mathrm{CDCl}_{3}\right): \delta[\mathrm{ppm}]=202.2 ; 159.1 ; 149.9 ; 130.3 ; 129.1 ; 128.7 ; 113.7 ; 72.9 ; 71.8 ; 66.9$; 55.2; 44.0; 39.4; 25.8; 18.2; 15.6; 14.2; -5.5. MS (EI, 70eV, 300 $\left.{ }^{\circ} \mathrm{C}\right): \mathrm{m} / \mathrm{z}=411\left(\mathrm{M}^{+}-18,14\right)$, 351 (12), 241 (13), 229 (19), $211(43), 121(100) .[\alpha]_{\mathrm{D}}^{20}=+16,3^{\circ}\left(\mathrm{c}=1,95 ; \mathrm{CHCl}_{3}\right)$. IR (Film) $1 / \lambda\left[\mathrm{cm}^{-1}\right]=2956 ; 2857 ; 1695 ; 1670 ; 1627 ; 1614 ; 1514 ; 1463 ; 1361 ; 1302 ; 1249 ; 1173 ;$ $1097 ; 1037 ; 837 ; 776$.

CBS- Reduktion of $\mathbf{2 6}$ to $\mathbf{2 7 , 2 8 , 2 9 .}$

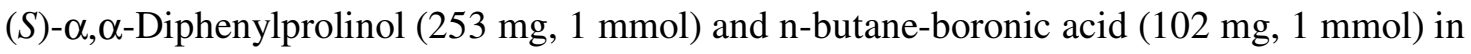
toluene $(40 \mathrm{~mL})$ were heated in a soxhlet apparatus filled with molecular sieves ( $4 \AA$ ) for $4 \mathrm{~h}$. The toluene was then removed by distillation until $15 \mathrm{~mL}$ were left and the remaining solution was cooled to $-80^{\circ} \mathrm{C}$. Catecholborane $(2.4 \mathrm{~g}, 20 \mathrm{mmol})$ in toluene $(10 \mathrm{~mL})$ was added and 26 (4.06 g, $10 \mathrm{mmol})$ in toluene $(20 \mathrm{~mL})$ was added dropwise and the mixture was stirred for $12 \mathrm{~h}$. Usual workup furnished after chromatography (hexanes/ethyl acetate 3 : 1) $27(2.64 \mathrm{~g}, 65 \%), \mathbf{2 8}(0.27 \mathrm{~g}, 7 \%)$ and $29(0.97 \mathrm{~g}, 24 \%)$ as colorless oils. For characterization 29 was reduced $\left(\mathrm{NaBH}_{4}\right.$ in $\left.\mathrm{MeOH}\right)$ to a mixture of 21 and 21 a.

(2R, 3S, 6R) 7-tert-Butyldimethylsilyloxy-1-p -methoxybenzyloxy -2,6-dimethyl-hept-4en-3-ol (27)

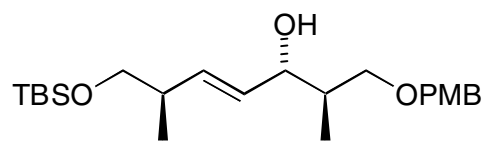

$\mathrm{R}_{\mathrm{f}}=0.35$ (hexanes/EtOAc $3: 1$ ), HPLC: $\mathrm{t}_{\mathrm{R}}=7.0 \mathrm{~min}(15 \%$ EtOAc in hexane). Anal. Calcd. for $\mathrm{C}_{23} \mathrm{H}_{40} \mathrm{O}_{4} \mathrm{Si}$ : C, 67.60; H, 9.87; found: C, 67.85; H, 9.85. ${ }^{1} \mathrm{H}-\mathrm{NMR}\left(400 \mathrm{MHz} ; \mathrm{CDCl}_{3}\right): \delta$ $[\mathrm{ppm}]=7.24(\mathrm{~d}, J=8.7 \mathrm{~Hz}, 2 \mathrm{H}), 6.87(\mathrm{~d}, J=8,7 \mathrm{~Hz}, 2 \mathrm{H}), 5.60\left(\mathrm{dd}, \mathrm{J}=15.6, \mathrm{CHCl}_{3}\right)$. IR (Film) $1 / \lambda[\mathrm{cm}-1]=3456,2954,2857,1613,1514,1463,1361,1302,1250,1173,1089,1037$, $973,836,776$. 
$(2 R, 3 R, 6 R)$ 7-tertButyldimethylsilyloxy-1-p-methoxybenzyloxy -2,6-dimethyl-hept-4en-3-ol (28)

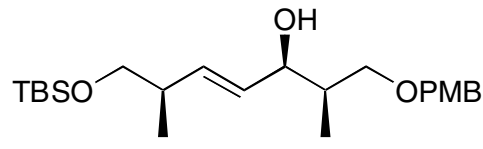

$\mathrm{R}_{\mathrm{f}}=0.35$ (Hexan/EtOAc $\left.3: 1\right)$. HPLC: $\mathrm{t}_{\mathrm{R}}=5.8 \mathrm{~min}(15 \%$ EtOAc in hexane). Anal. Calcd. for $\mathrm{C}_{23} \mathrm{H}_{40} \mathrm{O}_{4} \mathrm{Si}$ : C, 67.60; H, 9.87; found: C, 67.43; H, 9.64. ${ }^{1} \mathrm{H}-\mathrm{NMR}\left(400 \mathrm{MHz}, \mathrm{CDCl}_{3}\right.$ ): $\delta$ $[\mathrm{ppm}]=7.24(\mathrm{~d}, J=8.7 \mathrm{~Hz}, 2 \mathrm{H}), 6.87(\mathrm{~d}, J=8,7 \mathrm{~Hz}, 2 \mathrm{H}), 5.58(\mathrm{dd}, \mathrm{J}=15.6,6.9 \mathrm{~Hz}, 1 \mathrm{H}), 5.48$ (dd, 15.6, 7.0Hz, 1H), 4.42 (s, 2H), $4.15(\mathrm{~m}, 1 \mathrm{H}), 3.80(\mathrm{~s}, 3 \mathrm{H}), 3.49$ (dd, J = 9.7, 6.3Hz, 1H), $3.46(\mathrm{~m}, 2 \mathrm{H}), 3.39(\mathrm{dd}, \mathrm{J}=9.7,7.1 \mathrm{~Hz}, 1 \mathrm{H}), 2.85(\mathrm{~d}, \mathrm{~J}=5.1 \mathrm{~Hz}, 1 \mathrm{H}), 2.32(\mathrm{~m}, 1 \mathrm{H}), 2.03(\mathrm{~m}$, $1 \mathrm{H}), 0.99(\mathrm{~d}, \mathrm{~J}=6.7 \mathrm{~Hz}, 3 \mathrm{H}), 0.89$ (s, 9H), 0.87 (d, J=7.0Hz, 3H), 0.03 (s, 6H). ${ }^{13} \mathrm{C}-\mathrm{NMR}$ $\left(100.3 \mathrm{MHz} ; \mathrm{CDCl}_{3}\right): \delta[\mathrm{ppm}]=159.2,134.6,130.1,129.8,129.3,113.8,75.3,73.5,73.0$, $68.0,55.2,38.2,38.6,25.9,18.3,16.6,11.9,-5.4 .[\alpha]_{\mathrm{D}}^{20}=+1.2^{\circ}\left(\mathrm{c}=2.0 ; \mathrm{CHCl}_{3}\right)$.

butyldiphenylsilyloxy-2,6-dimethyl-9-hydroxy-5-p-methoxybenzyloxy-nondeca-12,14dien-7-one (31a)

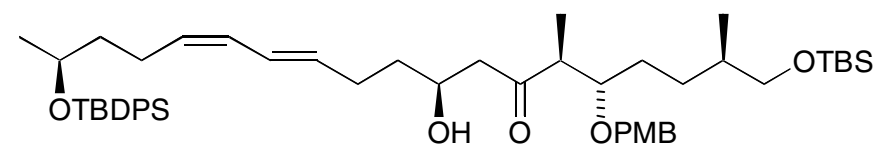

(-)-Chlorodiisopinocampheylborane $(545 \mathrm{mg}, 1.70 \mathrm{mmol})$ in THF $(5 \mathrm{~mL})$ was treated with triethylamine $(0.26 \mathrm{ml}, 1.83 \mathrm{mmol})$ at $-78^{\circ} \mathrm{C}$. Over $15 \mathrm{~min}$ ketone $5(690 \mathrm{mg}, 1.65 \mathrm{mmol})$ in THF ( $5 \mathrm{~mL}$ ) was added and the mixture was stirred at $-78^{\circ} \mathrm{C}$ for $30 \mathrm{~min}$. The mixture was then cooled to $-90^{\circ} \mathrm{C}$ and aldehyde $4 \mathbf{b}(540 \mathrm{mg}, 1.83 \mathrm{mmol})$ in THF $(5 \mathrm{~mL})$ was added via syringe pump over $2 \mathrm{~h}$. The mixture was stirred at $-78^{\circ} \mathrm{C}$ for another $\mathrm{h}$ and was then quenched with aqueous $\mathrm{NH}_{4} \mathrm{Cl}(20 \mathrm{~mL})$. The organic layer was concentrated under reduced pressure, diluted with pentane and treated with a mixture of $30 \%$ aqueous $\mathrm{H}_{2} \mathrm{O}_{2}(0.8 \mathrm{~mL}, 7$ $\mathrm{mmol})$ and $0.5 \mathrm{M} \mathrm{NaOH}(10 \mathrm{~mL})$ for $10 \mathrm{~min}$. The organic phase was washed with brine, dried $\left(\mathrm{MgSO}_{4}\right)$ and chromatographed (hexanes / EtOAc, $\left.3: 1, \mathrm{R}_{\mathrm{f}}=0.39\right)$ to obtain 31a and 31b (730 mg, $83 \%)$ in a ratio of $95: 5$ as a viscous oil. Anal. Calcd. for $\mathrm{C}_{51} \mathrm{H}_{78} \mathrm{O}_{6} \mathrm{Si}_{2}: \mathrm{C}$, 72.63; H, 9.32; found: C, 72,35; H, 9.54. ${ }^{1} \mathrm{H}-\mathrm{NMR}\left(400 \mathrm{MHz} ; \mathrm{CDCl}_{3}\right): \delta[\mathrm{ppm}]=7.69(\mathrm{~d}$, $\mathrm{J}=7.5 \mathrm{~Hz}, 4 \mathrm{H}), 7.32-7.44(\mathrm{~m}, 6 \mathrm{H}), 7.18(\mathrm{dt}, \mathrm{J}=8.4,2.5 \mathrm{~Hz}, 2 \mathrm{H}), 6.84(\mathrm{dt}, \mathrm{J}=.8,42.5 \mathrm{~Hz}, 2 \mathrm{H})$, $6.25(\mathrm{dd}, \mathrm{J}=14.8,10.9 \mathrm{~Hz}, 1 \mathrm{H}), 5.87$ (dd, J=10.9, 9.9Hz, 1H), 5.60 (dt, J=14.8, 6.9Hz, 1H), 
$5.18(\mathrm{dt}, \mathrm{J}=9.9,7.4 \mathrm{~Hz}, 1 \mathrm{H}), 4.44(\mathrm{~d}, \mathrm{~J}=10.8 \mathrm{~Hz}, 1 \mathrm{H}), 4.26(\mathrm{~d}, \mathrm{~J}=10.8 \mathrm{~Hz}, 1 \mathrm{H}), 3.99(\mathrm{~m}, 1 \mathrm{H})$, 3.87 (qt, J=6.9, 5.9Hz, 1H), $3.77(\mathrm{~s}, 3 \mathrm{H}), 3.64(\mathrm{~m}, 1 \mathrm{H}), 3.44(\mathrm{dd}, \mathrm{J}=9.8,5.6 \mathrm{~Hz}, 1 \mathrm{H}), 3.40$ $(\mathrm{dd}, \mathrm{J}=9.8,5.9 \mathrm{~Hz}, 1 \mathrm{H}), 3.16(\mathrm{~d}, 2.5 \mathrm{~Hz}, 1 \mathrm{H}), 2.83(\mathrm{dq}, \mathrm{J}=8.9,6.9 \mathrm{~Hz}, 1 \mathrm{H}), 2.67$ (dd, J=17.7, 2.5Hz, 1H), 2.49 (dd, J=17.7, 9.3Hz, 1H), 2.24-2.06 (m, 4H), $1.71(\mathrm{~m}, 2 \mathrm{H}), 1.60-1.36$ (m, 5H), 1.27 (m, 2H), 1.08 (d, J=6.9Hz, 3H), 1.06 (s, 9H), 0.99 (d, J=6.9Hz, 3H), 0.89 (s, 9H), $0.88(\mathrm{~d}, \mathrm{~J}=6.9 \mathrm{~Hz}, 3 \mathrm{H}), 0.04(\mathrm{~s}, 6 \mathrm{H})$.

${ }^{13} \mathrm{C}-\mathrm{NMR}\left(63 \mathrm{MHz} ; \mathrm{CDCl}_{3}\right): \delta[\mathrm{ppm}]=215.7 ; 159.3 ; 135.9 ; 134.8 ; 133.7 ; 130.0 ; 129.4$; $128.4 ; 127.5 ; 127.4 ; 126.0 ; 113.8 ; 81.2 ; 71.6 ; 69.2 ; 67.9 ; 67.0 ; 55.2 ; 50.3 ; 49.4 ; 39.4 ; 36.0$; $28.8 ; 27.6 ; 27.0 ; 25.9 ; 23.6 ; 23.2 ; 22.6 ; 19.3 ; 18.3 ; 16.9 ; 14.1 ; 12.8 ;-5.4$. MS : ( FD, 7kV, $3 \mathrm{~mA}): \mathrm{m} / \mathrm{z}=842[\mathrm{M}](100), 843[\mathrm{M}+1](71), 844[\mathrm{M}+2](26) .[\alpha]_{\mathrm{D}}^{20}=+22.2(\mathrm{c}=2.0$;

$\mathrm{CHCl}_{3}$ ). IR (Film) $1 / \lambda\left[\mathrm{cm}^{-1}\right]=3546,2955,2931,2857,1706,1613,1514,1472,1463$, $1428,1376,1362,1250,1110,1038,1007,837,776,703$.

\section{Methyl-(4S) 4,5-isopropylidene-dioxy-pentanoate}

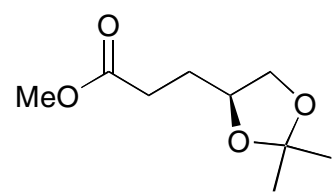

Methyl-(4S) 4,5-isopropylidene-dioxy-pent-2-enoate (4.5 g, $22.5 \mathrm{mmol})$ was hydrogenated with Raney nickel to give after chromatography (hexanes/EtOAc $\left.5: 1 \mathrm{R}_{\mathrm{f}}=0,43\right)$ methyl $(4 S)$ 4,5-isopropylidene-dioxy-pentanoate $(4.2 \mathrm{~g}, 93 \%)$ as a colorless oil. ${ }^{1} \mathrm{H}-\mathrm{NMR}(400 \mathrm{MHz}$; $\left.\mathrm{CDCl}_{3}\right): \delta[\mathrm{ppm}]=, 4.08(\mathrm{ddt}, \mathrm{J}=7.6,6.0,5.9 \mathrm{~Hz}, 1 \mathrm{H}), 3.99(\mathrm{dd}, \mathrm{J}=7.5,6.0 \mathrm{~Hz}, 1 \mathrm{H}), 3.63(\mathrm{~s}$, $3 \mathrm{H}), 3.50(\mathrm{dd}, \mathrm{J}=7.6,7.5 \mathrm{~Hz}, 1 \mathrm{H}), 2.32-3.47(\mathrm{~m}, 2 \mathrm{H}), 1.75-1.91(\mathrm{~m}, 2 \mathrm{H}), 1.35(\mathrm{~s}, 3 \mathrm{H}), 1.29$ $(\mathrm{s}, 3 \mathrm{H}) .{ }^{13} \mathrm{C}-\mathrm{NMR}\left(63 \mathrm{MHz} ; \mathrm{CDCl}_{3}\right): \delta[\mathrm{ppm}]=173.5,108.9,74.8,68.9,51.5,30.1,28.7$, 26.8, 25.5. MS (EI, 70eV, 20 C) m/z = 174 [M-28] (8), 173 [M-29] (100), 131 (21), 99 (68). $[\alpha]_{\mathrm{D}}^{20}=-1.0^{\circ}\left(\mathrm{c}=2.0 ; \mathrm{CHCl}_{3}\right) . \mathrm{IR}($ Film $) 1 / \lambda\left[\mathrm{cm}^{-1}\right]=2986,2952,2874,1740,1438,1379$, $1370,1256,1214,1171,1157,1075,858$.

(4S)-4,5-Isopropyliden-dioxy-pentanal (33)

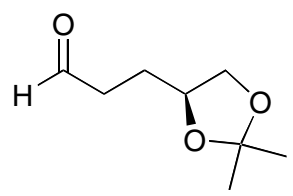

Methyl (4S) 4,5-isopropylidene-dioxy-pentanoate (2.0 g, $9.9 \mathrm{mmol})$ was reduced with DIBAL-H (TP 2) to give, after chromatography (hexanes/EtOAc $\left.5: 1 ; \mathrm{R}_{\mathrm{f}}=0,43\right) 33(1.45 \mathrm{~g}$, 
$93 \%)$ as a colorless oil. ${ }^{1} \mathrm{H}-\mathrm{NMR}\left(250 \mathrm{MHz} ; \mathrm{CDCl}_{3}\right): \delta=9.8(\mathrm{t}, \mathrm{J}=1.3 \mathrm{~Hz}, 1 \mathrm{H}), 4.14(\mathrm{~m}$, 1H), $4.04(\mathrm{dd}, \mathrm{J}=7.7,6.2 \mathrm{~Hz}, 1 \mathrm{H}), 3.56(\mathrm{~s}, 3 \mathrm{H})(\mathrm{dd}, \mathrm{J}=7.7,6.7 \mathrm{~Hz}, 1 \mathrm{H}), 2.54-2.64(\mathrm{~m}, 2 \mathrm{H})$, 1.78-2.01(m, 2H), $1.41(\mathrm{~s}, 3 \mathrm{H}), 1.34(\mathrm{~s}, 3 \mathrm{H}) .{ }^{13} \mathrm{C}-\mathrm{NMR}\left(63 \mathrm{MHz} ; \mathrm{CDCl}_{3}\right): \delta[\mathrm{ppm}]=201.5$, 109.1, 74.8, 69.0, 40.0, 26.8, 26.0, 25.5. MS: (EI, 70eV, 20 $\mathrm{C}): \mathrm{m} / \mathrm{z}=144[\mathrm{M}-15$ ] (7), 143 [M-15] (100), 101 (15), 83 (57), 72 (44). HRMS: calcd. for $\mathrm{C}_{7} \mathrm{H}_{11} \mathrm{O}_{3}$ : 143.0708; found: 143.0712. $[\alpha]_{\mathrm{D}}^{20}=-2.2^{\circ}\left(\mathrm{c}=2.0 ; \mathrm{CHCl}_{3}\right)$. IR (Film) $1 / \lambda\left[\mathrm{cm}^{-1}\right]=2987,2937,2875,1724$, $1379,1370,1248,1215,1157,1087,1058$.

\section{(1E, 5S)-1-Iodo-5,6-isopropyliden-dioxy-hex-1-ene (34)}

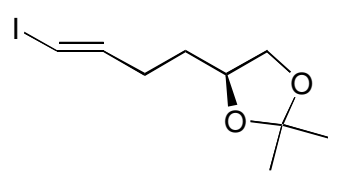

$\mathrm{CrCl}_{3}(1.27 \mathrm{~g}, 8 \mathrm{mmol})$ in THF $(15 \mathrm{~mL})$ was treated with $1 \mathrm{M} \mathrm{LAH}$ in THF ( $\left.3 \mathrm{~mL}, 3 \mathrm{mmol}\right)$ and stirred for $2 \mathrm{~h}$. The black suspension was cooled to $0^{\circ} \mathrm{C}$ and triiodomethane (394 $\mathrm{mg}, 1$ $\mathrm{mmol}$ ) and 23 ( $80 \mathrm{mg}, 0.5 \mathrm{mmol}$ ) in THF (20 mL) were added. After $2 \mathrm{~h}$ the mixture was quenched with water $(30 \mathrm{~mL})$ and worked up with ether to give, after chromatography (hexanes/ethyl acetate $\left.5: 1 ; \mathrm{R}_{\mathrm{f}}=0.52\right) 34(85 \mathrm{mg}, 60 \%)$ as a 3:1- $E / Z$-mixture from which the $E$-isomer was separated by HPLC (10\% EtOAc in hexane, $\left.\mathrm{t}_{\mathrm{R}}=4.2 \mathrm{~min}\right) .{ }^{1} \mathrm{H}-\mathrm{NMR}(400$ $\left.\mathrm{MHz} ; \mathrm{CDCl}_{3}\right): \delta=6.52(\mathrm{dt}, \mathrm{J}=14.5,7.0 \mathrm{~Hz}, 1 \mathrm{H}), 6.05(\mathrm{~d}, \mathrm{~J}=14.5 \mathrm{~Hz}, 1 \mathrm{H}), 4.07(\mathrm{~m}, 1 \mathrm{H})$, 4.02 (dd, J =7.5, 6.0 Hz, 1H), 3.50 (dd, J =7.5, 7.0 Hz, 1H), 2.06-2.25 (m, 2H), 1.56-1.75(m, 2H), 1.39 (s, 3H), 1.34 (s, 3H). ${ }^{13} \mathrm{C}-\mathrm{NMR}\left(100.3 \mathrm{MHz} ; \mathrm{CDCl}_{3}\right): \delta[\mathrm{ppm}]==145.4,108.9$, 75.3, 75.0, 69.2, 32.3, 26.9, 25.6. MS: (EI, 70eV, 40 $): \mathrm{m} / \mathrm{z}=282$ [M] (3), 267 [M-15] (32), 167 (13), 97 (21), 80 (34). $\alpha^{20}{ }_{D}=+0.55^{\circ}$ (c =2.0; $\mathrm{CHCl}_{3}$ ). IR (Film) $1 / \lambda\left[\mathrm{cm}^{-1}\right]=2984$, 2935, 2871, 1453, 1378, 1370, 1216, 1154, 1069.

$(2 S, 11 R)$-11-tert-Butyldiphenylsilyloxy -1,2-isopropyliden-dioxydodec-5-en -7-yne (35)

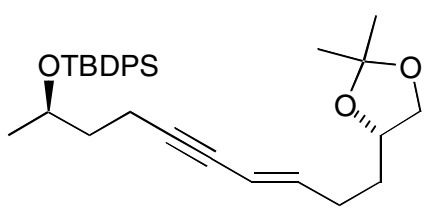


$34(1.4 \mathrm{~g}, 5.0 \mathrm{mmol})$ and $9 \mathrm{~b}(1.85 \mathrm{~g}, 5.5 \mathrm{mmol})$ in benzene $(30 \mathrm{~mL})$ were stirred at $22^{\circ} \mathrm{C}$ with butylamine (5 mL, $50 \mathrm{mmol}), \mathrm{CuI}(280 \mathrm{mg}, 1.5 \mathrm{mmol})$ and tetrakistriphenylposphinepalladium (580 mg, $0.5 \mathrm{mmol})$ for $12 \mathrm{~h}$ and then quenched with sat. $\mathrm{NH}_{4} \mathrm{Cl}(10 \mathrm{~mL})$. Usual workup delivered after chromatography $\left(\mathrm{R}_{\mathrm{f}}=0,67\right.$, hexanes/ethyl acetate 5:1) 35 (2.1 g, 86 $\%$ ) as a colorless oil. Anal. Calcd. for $\mathrm{C}_{31} \mathrm{H}_{42} \mathrm{O}_{3} \mathrm{Si}$ : C, 75.87; $\mathrm{H}, 8.63$; found: $\mathrm{C}, 75.38 ; \mathrm{H}$, 7.94. ${ }^{1} \mathrm{H}-\mathrm{NMR}\left(400 \mathrm{MHz} ; \mathrm{CDCl}_{3}\right): \delta[\mathrm{ppm}]=7.66-7.73(\mathrm{~m}, 4 \mathrm{H}), 7.34-7.44(\mathrm{~m}, 6 \mathrm{H}),, 5.98$ (dt, J=15.6, 7.0 Hz, 1H), 5.46 (dt, J=15.6, $1.5 \mathrm{~Hz}, 1 \mathrm{H}), 4.11$ (ddt, J=7.6, 6.0, 5.9 Hz, 1H), $4.04(\mathrm{dd}, \mathrm{J}=7.5,6.0 \mathrm{~Hz}, 1 \mathrm{H}), 3.91(\mathrm{qt}, \mathrm{J}=6.1,5.8 \mathrm{~Hz}, 1 \mathrm{H}), 3.52(\mathrm{dd}, \mathrm{J}=7.6,7.5 \mathrm{~Hz}, 1 \mathrm{H}), 2.36$ $(\mathrm{dd}, J=7.5,7.0 \mathrm{~Hz}, 2 \mathrm{H}), 2.22(\mathrm{~m}, 1 \mathrm{H}), 2.15(\mathrm{~m}, 1 \mathrm{H}), 1.54-1.79(\mathrm{~m}, 4 \mathrm{H}), 1.42(\mathrm{~s}, 3 \mathrm{H}), 1.36$ (s, 3H), 1.06 (s, 9H), 1.05 (d, J=5.9 Hz, 3H). ${ }^{13} \mathrm{C}-\mathrm{NMR}\left(63 \mathrm{MHz} ; \mathrm{CDCl}_{3}\right): \delta[\mathrm{ppm}]=141.7$, $135.9,134.8,134.2,129.5,129.4,127.4,110.7,108.8,89.0,78.9,75.3,69.3,68.5,38.4$, 32.8, 29.1, 27.0, 25.7, 23.1, 19.3, 15.5. MS: (EI, 70eV, $\left.100^{\circ} \mathrm{C}\right): \mathrm{m} / \mathrm{z}=575[\mathrm{M}-15]$ (4), 433 (22), 375 (42), 199 (100), 183 (12). HRMS: calcd. for $\mathrm{C}_{30} \mathrm{H}_{39} \mathrm{O}_{3} \mathrm{Si}$ : 475.2668 ; found: 475.2685. $[\alpha]_{\mathrm{D}}^{20}=-14,3^{\circ}\left(\mathrm{c}=2.0 ; \mathrm{CHCl}_{3}\right)$. IR (Film) $1 / \lambda\left[\mathrm{cm}^{-1}\right]=2932,2858,1428,1378$, 1214, 1137, 1110, 1025, 823, 703 .

\section{(2S, 5E, 7Z, 11R)-11-tert-Butyldimethylsilyloxy-1,2-isopropyliden-dioxydodeca-5,7-}

diene (36)

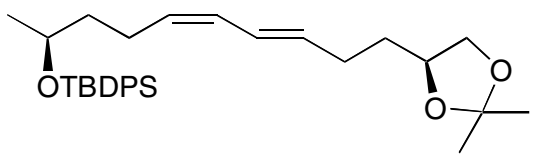

As described for 12a, 35 (2.4 g, $4.9 \mathrm{mmol})$ was hydrogenated to give $\mathbf{3 6}(2.15 \mathrm{~g}, 90 \%)$ after chromatography (hexanes/ethyl acetate 5:1, $\mathrm{R}_{\mathrm{f}}=0,67$ ). as a colorless oil. Anal. Calcd. for $\mathrm{C}_{31} \mathrm{H}_{44} \mathrm{O}_{3} \mathrm{Si}: \mathrm{C}, 75.56 ; \mathrm{H}, 9.00$; found: $\mathrm{C}, 74.66 ; \mathrm{H}, 8.79 .{ }^{1} \mathrm{H}-\mathrm{NMR}\left(400 \mathrm{MHz} ; \mathrm{CDCl}_{3}\right.$ ): $\delta$ $[\mathrm{ppm}]=7.72(\mathrm{dt}, \mathrm{J}=8.3,1.5 \mathrm{~Hz}, 4 \mathrm{H}), 7.33-7.44(\mathrm{~m}, 6 \mathrm{H}), 6.29(\mathrm{dd}, \mathrm{J}=15.2,10.9 \mathrm{~Hz}, 1 \mathrm{H})$, $5.91(\mathrm{dd}, \mathrm{J}=10.9,10.8 \mathrm{~Hz}, 1 \mathrm{H}), 5.65$ (dt, J=15.2, $6.9 \mathrm{~Hz}, 1 \mathrm{H}), 5.23(\mathrm{dt}, \mathrm{J}=10.8,7.3 \mathrm{~Hz}, 1 \mathrm{H})$, $4.11(\mathrm{ddt}, \mathrm{J}=7.2,6.2,6.1 \mathrm{~Hz}, 1 \mathrm{H}), 4.05$ (dd, J=7.6, 6.1 Hz, 1H), 3.91(qt, J=6.1, $5.9 \mathrm{~Hz}, 1 \mathrm{H})$, 3.53(dd, J=7.6, 7.2 Hz, 1H), 2.08-2.28 (m, 4H),1.78 (m, 1H), $1.61(\mathrm{~m}, 2 \mathrm{H}), 1.58(\mathrm{~m}, 1 \mathrm{H})$, 1.44 (s, 3H), 1.38 (s, 3H), 1.10 (d, J=5.9 Hz, 3H), 1.09 (s, 9H). ${ }^{13} \mathrm{C}-\mathrm{NMR}\left(63 \mathrm{MHz} ; \mathrm{CDCl}_{3}\right)$ : $\delta[\mathrm{ppm}]=135.9 ; 135.8 ; 134.8 ; 134.5 ; 133.1 ; 130.2 ; 129.4 ; 128.3 ; 127.5 ; 127.4 ; 126.2$; 
$\left.=1 ; \mathrm{CHCl}_{3}\right) . \mathrm{MS}:\left(\mathrm{EI}, 70 \mathrm{eV}, 70^{\circ} \mathrm{C}\right): \mathrm{m} / \mathrm{z} 478$ [M-14] (4), 436 [M-58] (6), 378 (24), 281

(26), 236 (23), 199 (100), 161 (85). HRMS: calcd. for $\mathrm{C}_{30} \mathrm{H}_{41} \mathrm{O}_{3} \mathrm{Si}$ : 477.2825; found :

477.2801. IR $1 / \lambda\left[\mathrm{cm}^{-1}\right]=2932,2858,1428,1378,1214,1132,1111,1070,823,740,703$.

\section{S, 5E, 7Z, 11R)-11-tert-Butyldimethylsilyloxy-dodeca-5,7-dien-1,2-diol}

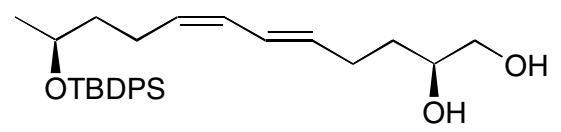

$36(2.51 \mathrm{~g}, 5.1 \mathrm{mmol})$ in $\mathrm{THF} /$ water $(25 \mathrm{~mL})$ was treated with TFA $(1 \mathrm{~mL})$ at $0^{\circ} \mathrm{C}$ and the mixture was stirred for $24 \mathrm{~h}$ at room temperature. Usual workup furnished, after chromatography (hexanes/ethyl acetate $\left.1: 1 ; \mathrm{R}_{\mathrm{f}}=0,21\right)(2 S, 5 E, 7 Z, 11 R)$-11-tertbutyldimethylsilyloxy-dodeca-5,7-dien-1,2-diol (1.68 g ,73\%) as a colorless oil, Anal. calcd. for $\mathrm{C}_{28} \mathrm{H}_{40} \mathrm{O}_{3} \mathrm{Si}: \mathrm{C}, 77.37$; H, 8.81; found: C, 68.03; H, 11.22. ${ }^{1} \mathrm{H}-\mathrm{NMR}$ (400 MHz; $\left.\mathrm{CDCl}_{3}\right): \delta[\mathrm{ppm}]=7.72(\mathrm{dt}, \mathrm{J}=8.3,1.5 \mathrm{~Hz}, 4 \mathrm{H}), 7.33-7.44(\mathrm{~m}, 6 \mathrm{H}), 6.29(\mathrm{dd}, \mathrm{J}=15.2,10.8$

Hz, 1H), 5.89 (dd, J=10.8, 10.3 Hz, 1H), 5.64 (dt, J=15.2, $6.9 \mathrm{~Hz}, 1 \mathrm{H}), 5.21$ (dt, J=10.3, 7.9 $\mathrm{Hz}, 1 \mathrm{H}), 3.90(\mathrm{qt}, \mathrm{J}=6.4,5.9 \mathrm{~Hz}, 1 \mathrm{H}), 3.73$ (tdd, J=7.4, 6.7, $2.9 \mathrm{~Hz}, 1 \mathrm{H}), 3.65$ (dd, J=11.3, 2.9 Hz, 1H), 3.45(dd, J=11.3, 7.4 Hz, 1H), 2.69 (s, 2H), 2.08-2.30 (m, 4H), 1.45-1.63 (m, 4H), 1.09 (d, J=6.4 Hz, 3H), 1.08 (s, 9H). ${ }^{13} \mathrm{C}-\mathrm{NMR}\left(63 \mathrm{MHz} ; \mathrm{CDCl}_{3}\right): \delta[\mathrm{ppm}]=135.9 ; 135.8$; $134.8 ; 134.5 ; 133.3 ; 130.3 ; 129.4 ; 128.3 ; 127.5 ; 127.4 ; 126.3 ; 71.7 ; 69.2 ; 66.7 ; 39.3 ; 32.7$; 28.9; 27.0; 23.6; 23.1; 19.2. MS: (EI, 70eV, 70 C): m/z = 422 [M-30] (1), 378 (3), 317 (6), 241 (6), 239 (8), 199 (100), 181 (23), 161 (39), 135 (39). HRMS: calcd. for $\mathrm{C}_{27} \mathrm{H}_{38} \mathrm{O}_{2} \mathrm{Si}$ : 422.2641; found: 422.2665. $[\alpha]_{\mathrm{D}}^{20}=-21,3^{\circ}\left(\mathrm{c}=2.0 ; \mathrm{CHCl}_{3}\right)$. IR (Film) $1 / \lambda\left[\mathrm{cm}^{-1}\right]=3368$, $2930,2858,1428,1428,1376,1132,1110,950,822,740,703$.

\section{$(2 S, 5 E, 7 Z, 11 R)-11$-tert-Butyldimethylsilyloxy-dodeca-5,7-dien-1,2-oxirane (37)}

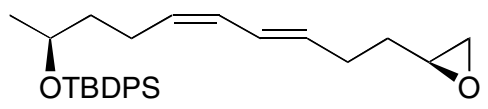

Sodium hydride $(60 \%, 88 \mathrm{mg}, 2,2 \mathrm{mmol})$ in DMF (5 mL) was treated dropwise with . $2 S$, $5 E, 7 Z, 11 R)$-11-tert-butyldimethylsilyloxy-dodeca-5,7-dien-1,2-diol (452 mg, $1.0 \mathrm{mmol}$ ) for $4 \mathrm{~h}$. The mixture was cooled to $-10^{\circ} \mathrm{C}$ and tosylimidazole $(250 \mathrm{mg}, 1.1 \mathrm{mmol}$ ) was added and the mixture was allowed to warm to $0^{\circ} \mathrm{C}$ for $30 \mathrm{~min}$. After stirring for another $30 \mathrm{~min}$ at $0^{\circ} \mathrm{C}$ 
the mixture was quenched with aqueous $\mathrm{NH}_{4} \mathrm{Cl}$. Usual workup including chromatography (hexanes/ethyl acetate $\left.1: 1 ; \mathrm{R}_{\mathrm{f}}=0.69\right)$ furnished $37(287 \mathrm{mg}, 66 \%)$ as a colorless oil. Anal. Calcd. for $\mathrm{C}_{28} \mathrm{H}_{38} \mathrm{O}_{2} \mathrm{Si}$ : C, 77.37; H, 8.81; found: C, 77.15; H, 8.70. ${ }^{1} \mathrm{H}-\mathrm{NMR}(400 \mathrm{MHz}$; $\left.\mathrm{CDCl}_{3}\right): \delta[\mathrm{ppm}]=7.72(\mathrm{dt}, \mathrm{J}=8.3,1.5 \mathrm{~Hz}, 4 \mathrm{H}), 7.33-7.44(\mathrm{~m}, 6 \mathrm{H}), 6.31(\mathrm{dd}, \mathrm{J}=14.5,11.3$ Hz, 1H), 5.91 (dd, J=11.3, 10.3 Hz, 1H), 5.67 (dt, J=14.5, $6.9 \mathrm{~Hz}, 1 \mathrm{H}), 5.23$ (dt, J=10.3, 7.9 $\mathrm{Hz}, 1 \mathrm{H}), 3.91(\mathrm{qt}, \mathrm{J}=6.2,5.9 \mathrm{~Hz}, 1 \mathrm{H}), 2.95$ (tdd, J=5.5, 4.4, $2.8 \mathrm{~Hz}, 1 \mathrm{H}), 2.76$ (dd, J=5.0, 4.4 $\mathrm{Hz}, 1 \mathrm{H}), 2.50$ (dd, J=5.0, $2.8 \mathrm{~Hz}, 1 \mathrm{H}), 2.10-2.32$ (m, 4H), 1.44-1.68 (m, 4H), 1.10 (d, J=5.9 $\mathrm{Hz}, 3 \mathrm{H}), 1.09$ (s, 9H). ${ }^{13} \mathrm{C}-\mathrm{NMR}\left(100,6 \mathrm{MHz} \mathrm{CDCl}_{3}\right): \delta[\mathrm{ppm}]=135.9 ; 135.8 ; 134.8 ; 134.5$; $132.8 ; 130.3 ; 129.4 ; 128.3 ; 127.5 ; 127.4 ; 126.3 ; 69.2 ; 51.8 ; 47.1 ; 39.3 ; 32.7 ; 29.3 ; 27.0 ;$ 23.6; $23.2 ; 19.3$.

MS (EI, 70eV, 60 C): m/z = 377 [M-57] (9), 281 (4), 239 (8), 199 (100) 183 (22), 161 (31). HRMS calcd. for $\mathrm{C}_{24} \mathrm{H}_{29} \mathrm{O}_{2} \mathrm{Si}$ : 377.1937; found, 377.1922. $[\alpha]_{\mathrm{D}}^{20}=-23.0^{\circ}\left(\mathrm{c}=2.0 ; \mathrm{CHCl}_{3}\right)$. IR $1 / \lambda\left[\mathrm{cm}^{-1}\right]=2952,2896,2856,1613,1514,1462,1422,1384,1302,1249,1172,1090$, $1040,1005,836$.

$(2 R, 3 S, 6 R)$ 7-tert-Butyldimethylsilyloxy-2,6-dimethyl-3-p-methoxybenzyloxy-1,1(propan-1',36-dithio)-heptane (38)

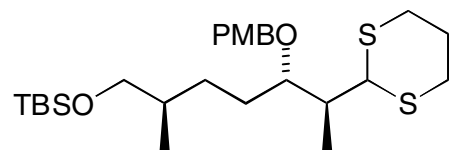

24 (820 mg, $2.0 \mathrm{mmol}$ ) and 1,3-bis-trimethylsilylthiylpropane (500 mg, $1.1 \mathrm{mmol}$ ) in $\mathrm{Et}_{2} \mathrm{O}$ $(20 \mathrm{~mL})$ were treated with zinc diiodide $(20 \mathrm{mg})$ at room temperature for $12 \mathrm{~h}$ and the product was directly purified by chromatography (hexanes/EtOAc $5: 1 ; \mathrm{R}_{\mathrm{f}}=0.69$ ). The product was stirred in $\mathrm{Et}_{2} \mathrm{O}(5 \mathrm{~mL})$ with additional zinc diiodide $(20 \mathrm{mg})$ for $12 \mathrm{~h}$.

Chromatography (hexanes/EtOAc $\left.5: 1 ; \mathrm{R}_{\mathrm{f}}=0.53\right)$ furnished $38(600 \mathrm{mg}, 60 \%)$ as a colorless oil. Anal. Calcd. for $\mathrm{C}_{26} \mathrm{H}_{46} \mathrm{O}_{3} \mathrm{~S}_{2} \mathrm{Si}$ : C, 62.60; H, 9.29; S, 12.86; found: C, 62.39; H, 9.66; S, 11.93. ${ }^{1} \mathrm{H}-\mathrm{NMR}\left(250 \mathrm{MHz} ; \mathrm{CDCl}_{3}\right): \delta=7.29(\mathrm{~d}, \mathrm{~J}=8.7 \mathrm{~Hz}, 2 \mathrm{H}), 6.88(\mathrm{~d}, \mathrm{~J}=8.7 \mathrm{~Hz}$, 2H),4.50 (d, J=10.8 Hz, 1H),4.45 (d, J=10.8 Hz, 1H), 4.44 (d, J=4.5 Hz, 1H), 3.81 (s, 3H), 3.57 (ddd, J=8.3, 6.2, 3.5 Hz, 1H), 3.45 (dd, J=9.9, 5.8 Hz, 1H), 3.37 (dd, J=9.9, 6.6 Hz,1H), $2.92(\mathrm{td}, \mathrm{J}=12.3,2.5 \mathrm{~Hz}, 1 \mathrm{H}), 2.86-2.75(\mathrm{~m}, 3 \mathrm{H}), 2.1(\mathrm{~m}, 2 \mathrm{H}), 1.83(\mathrm{~m}, 1 \mathrm{H}), 1.69(\mathrm{~m}, 1 \mathrm{H})$, 1.58-1.35 (m, 3H), 1.15 (m, 1H), 1.04 (d, J=6.9 Hz, 3H), 0.89 (s, 9H), 0.88 (d, J=6.0 Hz, 
$3 \mathrm{H}), 0.03(\mathrm{~s}, 6 \mathrm{H}) .{ }^{13} \mathrm{C}-\mathrm{NMR}\left(63 \mathrm{MHz} ; \mathrm{CDCl}_{3}\right): \delta[\mathrm{ppm}]=159.1 ; 131.1 ; 129.4 ; 113.7 ; 79.5$;

71.6; 68.1; 55.2; 52.3; 41.5; 36.0; 31.4; 30.7; 27.6; 26.4; 25.9; 18.3; 17.0; 12.6; -5.4. MS:

$\left(\mathrm{FI} 90^{\circ} \mathrm{C}\right) \mathrm{m} / \mathrm{z}=498[\mathrm{M}](100)$. HRMS (EI, $70 \mathrm{eV}$ ) calcd. for $\mathrm{C}_{26} \mathrm{H}_{46} \mathrm{O}_{3} \mathrm{~S}_{2} \mathrm{Si}: 498.2658$;

found: 498.2639. $[\alpha]_{\mathrm{D}}^{20}=+14.9^{\circ}\left(\mathrm{c}=2.0 ; \mathrm{CHCl}_{3}\right)$. IR (Film) $1 / \lambda\left[\mathrm{cm}^{-1}\right]=2953,2931,2896$, 2856, 1613, 1514, 1463, 1302, 1249, 1172, 1089, 1038, 837, 776.

$(2 R, 5 S, 6 R, 9 S, 12 E, 14 Z, 18 S)$ 1-tert-Butyldimethylsilyloxy-18-tert-butyldiphenylsilyloxy 2,6-dimethyl-9-hydroxy-5-p-methoxybenzyloxy-7,7( propane-1',3‘-dithio)nonadeca-12,14-diene (39)

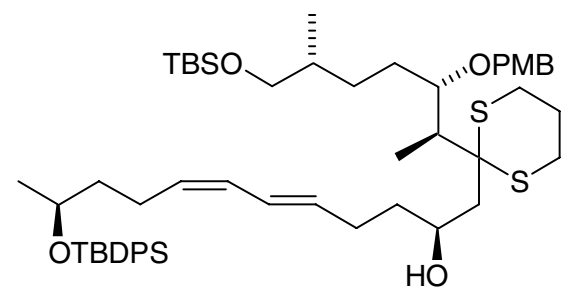

$38(5 \mathrm{mg}, 0.15 \mathrm{mmol})$ in THF $(0.75 \mathrm{~mL})$ was treated at $-60^{\circ} \mathrm{C}$ with TMEDA $(31.5 \mu \mathrm{L}, 0.2$ $\mathrm{mmol})$ and over $15 \mathrm{~min}$ with $1.2 \mathrm{M}$ s-butyl-lithium in hexane $(150 \mu \mathrm{L}, 0.18 \mathrm{mmol})$. Within 1 $\mathrm{h}$ the mixture was allowed to warm to $-40^{\circ} \mathrm{C}$ and then DMPU $(55 \mu \mathrm{L})$ was added. After $1 \mathrm{~h}$, $37(65 \mathrm{mg}, 0.15 \mathrm{mmol})$ in THF $(0.65 \mathrm{~mL})$ was added at $-40^{\circ} \mathrm{C}$, the mixture was stirred at $25^{\circ} \mathrm{C}$ for $3 \mathrm{~h}$ and then quenched with satd. $\mathrm{NH}_{4} \mathrm{Cl}$. Usual workup furnished after chromatography (hexanes/ethyl acetate $\left.5: 1 ; \mathrm{R}_{\mathrm{f}}=0.43\right) \mathbf{3 9}(68 \mathrm{mg}, 40 \%)$ as a colorless oil along with starting materials (60 mg). Anal. calcd. for $\mathrm{C}_{54} \mathrm{H}_{84} \mathrm{O}_{5} \mathrm{~S}_{2} \mathrm{Si}_{2}: \mathrm{C}, 69.47 ; \mathrm{H}, 9.07 ; \mathrm{S}$, 6.87; found: C, 69.30; C, 8.80; S, 7.09. ${ }^{1} \mathrm{H}-\mathrm{NMR}\left(400 \mathrm{MHz} ; \mathrm{CDCl}_{3}\right): \delta[\mathrm{ppm}]=7.69(\mathrm{~d}$, $\mathrm{J}=8.4 \mathrm{~Hz}, 4 \mathrm{H}), 7.36-7.44(\mathrm{~m}, 6 \mathrm{H}), 7.30(\mathrm{dt}, \mathrm{J}=8.7,2.0 \mathrm{~Hz}, 2 \mathrm{H}), 6.87(\mathrm{dt}, \mathrm{J}=.8,7,2.0 \mathrm{~Hz}, 2 \mathrm{H})$, 6.29 (dd, J=14.6, 10.9 Hz, 1H), 5.90 (dd, J=10.9, 10.7 Hz, 1H), 5.66 (dt, J=14.6, 6.9 Hz, 1H), 5.18 (dt, J=10.7, 7.6 Hz, 1H), 4.54 (d, J=11.3 Hz, 1H), 4.45 (d, J=11.3 Hz, 1H), 3.96(m, 2H), 3.88(qt, J=6.9, $5.9 \mathrm{~Hz}, 1 \mathrm{H}), 3.79$ (s, 3H), 3.49 (dd, J=9.8, 5.4 Hz, 1H), 3.40 (dd, J=9.8, $6.4 \mathrm{~Hz}, 1 \mathrm{H}), 3.11$ (ddd, J=14.7, 10.8, 2.6 Hz, 1H), 2.94 (ddd, J=14.7, 12.3, $2.5 \mathrm{~Hz}, 1 \mathrm{H}), 2.73$ (m, 2H), $2.48(\mathrm{qd}, \mathrm{J}=7.0,2.4 \mathrm{~Hz}, 1 \mathrm{H}), 2.40(\mathrm{dd}, \mathrm{J}=15.6,8.8 \mathrm{~Hz}, 1 \mathrm{H}), 2.06-2.30(\mathrm{~m}, 5 \mathrm{H}), 1.84$ (m, 2H),1.69-1.36 (m, 7H), $1.28(\mathrm{~m}, 2 \mathrm{H}), 1.08$ (d, J=6.9 Hz, 3H), 1.07 (s, 9H), 1.07 (d, J=6.9 $\mathrm{Hz}, 3 \mathrm{H}), 0.89$ (s, 9H), 0.88 (d, J=6.9 Hz, 3H), 0.04 (s, 6H). ${ }^{13} \mathrm{C}-\mathrm{NMR}\left(100 \mathrm{MHz} ; \mathrm{CDCl}_{3}\right): \delta$ $[\mathrm{ppm}]=159.1 ; 135.9 ; 135.8 ; 134.8 ; 134.5 ; 133.9 ; 130.9 ; 129.9 ; 129.5 ; 129.4 ; 125.9 ; 113.7$; $80.4 ; 71.3 ; 69.2 ; 68.3 ; 67.8 ; 55.9 ; 55.2 ; 42.8 ; 39.4 ; 37.7 ; 35.9 ; 30.1 ; 29.8 ; 28.9 ; 27.0 ; 25.7$; $24.9 ; 26.0 ; 25.8 ; 24.9 ; 23.6 ; 23.2 ; 19.3 ; 18.3 ; 17.0 ; 9.7 ;-5.4 .[\alpha]_{\mathrm{D}}^{20}=-19,5^{\circ}\left(\mathrm{c}=2 ; \mathrm{CHCl}_{3}\right)$. 
IR (Film) $1 / \lambda\left[\mathrm{cm}^{-1}\right]=3462,2954,2930,2856,1612,1514,1462,1428,1376,1302,1249$, 1172, 1110, 1037, 1007, 950, 837, 776, 704.

39 (60 mg, $0.063 \mathrm{mmol})$ in acetone/water $(4: 1,2 \mathrm{~mL})$ was treated with NBS (0.18 $\mathrm{mg}, 1.0$ $\mathrm{mmol})$ and silver perchlorate $(207 \mathrm{mg}, 1.0 \mathrm{mmol})$ for $10 \mathrm{~s}$. The product was isolated by extraction with ether and then purified by chromatography (hexanes / EtOAc, $3: 1, \mathrm{R}_{\mathrm{f}}=$ 0.39 ) to give 31a (47 mg, $90 \%$ ), identical with the compound described above.

$(2 R, 5 S, 6 R, 9 S, 12 E, 14 Z, 18 S)$ 1-tert-Butyldimethylsilyloxy-18- tertbutyldiphenylsilyloxy 2,6-dimethyl-5,9-di-p-methoxybenzyloxy-nonadeca-12,14-dien-7one

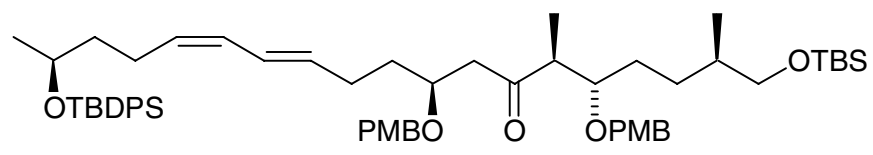

31a ( $610 \mathrm{mg}, 0.72 \mathrm{mmol}$ ) was treated (TP 6) with $p$-methoxybenzyl-trichloracetimidate $(600 \mathrm{mg}, 2.1 \mathrm{mmol})$ in cyclohexane $/ \mathrm{CH}_{2} \mathrm{Cl}_{2}(6 \mathrm{~mL})(5: 1)$ to obtain the PMB ether $(575 \mathrm{mg}$, $83 \%$ ) after chromatography (hexanes/EtOAc $5: 1 ; \mathrm{R}_{\mathrm{f}}=0.37$ ) as a colorless oil. ${ }^{1} \mathrm{H}-\mathrm{NMR}$ $\left(400 \mathrm{MHz} ; \mathrm{CDCl}_{3}\right): \delta[\mathrm{ppm}]=7.70(\mathrm{~d}, \mathrm{~J}=7.4 \mathrm{~Hz}, 4 \mathrm{H}), 7.32-7.44(\mathrm{~m}, 6 \mathrm{H}), 7.22(\mathrm{~d}, \mathrm{~J}=8.4 \mathrm{~Hz}$, 2H), 7.19 (d, J=8.4Hz, 2H), 6.85 (d, 8.4Hz, 2H), 6.84 (d, J=.8,4Hz, 2H), 6.25 (dd, J=15.0, $10.9 \mathrm{~Hz}, 1 \mathrm{H}), 5.88(\mathrm{dd}, \mathrm{J}=10.9,9.7 \mathrm{~Hz}, 1 \mathrm{H}), 5.59(\mathrm{dt}, \mathrm{J}=15.0,7.0 \mathrm{~Hz}, 1 \mathrm{H}), 5.19$ (dt, J=9.7, $7.4 \mathrm{~Hz}, 1 \mathrm{H}), 4.43(\mathrm{~d}, \mathrm{~J}=10.8 \mathrm{~Hz}, 1 \mathrm{H}), 4.42(\mathrm{~s}, 2 \mathrm{H}), 4.31$ (d, J=10.8Hz, 1H), 3.96(m, 1H), 3.89(qt, J=6.9, 5.9Hz,1H), 3.79 (s, 3H), 3.77(s, 3H) 3.67 (m, 1H), 3.45 (dd, J=9.6, 5.2Hz, $1 \mathrm{H}), 3.40(\mathrm{dd}, \mathrm{J}=9.6,6.1 \mathrm{~Hz}, 1 \mathrm{H}), 2.85(\mathrm{~m}, 2 \mathrm{H}), 2.53(\mathrm{dd}, \mathrm{J}=17.0,5.1 \mathrm{~Hz}, 1 \mathrm{H}), 2.24-2.06$ (m, 4H), $1.71(\mathrm{~m}, 2 \mathrm{H}), 1.60-1.36(\mathrm{~m}, 5 \mathrm{H}), 1.27(\mathrm{~m}, 2 \mathrm{H}), 1.08$ (d, J=6.9Hz, 3H), 1.07 (s, 9H), 1.00 $(\mathrm{d}, \mathrm{J}=6.9 \mathrm{~Hz}, 3 \mathrm{H}), 0.90$ (s, 9H), 0.88 (d, J=6.8Hz, 3H), 0.04 (s, 6H). ${ }^{13} \mathrm{C}-\mathrm{NMR}(63 \mathrm{MHz}$; $\left.\mathrm{CDCl}_{3}\right): \delta[\mathrm{ppm}]=212.5,159.1,135.9,135.8,134.8,134.5,133.8,130.6,129.9,129.5$, $129.4,129.3,128.5,127.5,127.4,125.9,113.7,80.7,71.6,71.5,69.2,68.0,55.2,59.9,48.8$, $39.4,36.0,34.5,30.3,29.7,28.7,27.5,27.0,25.9,23.6,23.1,19.3,18.3,16.9,14.1,12.8$, 5.4. MS : ( FD, 7kV, 3mA) :m/z = 963.5 [M+1] (28), 962.7 [M] (100), 945 [M-18] (31). $[\alpha]_{\mathrm{D}}^{20}=+6.2^{\circ}\left(\mathrm{c}=0.50 ; \mathrm{CHCl}_{3}\right) . \mathrm{IR}($ Film $) 1 / \lambda\left[\mathrm{cm}^{-1}\right]=2955,2931,2857,1713,1613$, $1514,1463,1428,1376,1361,1302,1249,1173,1110,1086,1038,1007,836,822,776$, 703. 
(2R, 5S, 6R, 9S, 12E, 14Z, 18S) 18- tertButyldiphenylsilyloxy 2,6-dimethyl-5,9-di-pmethoxybenzyloxy-1-hydroxy-nonadeca-12,14-dien-7-one

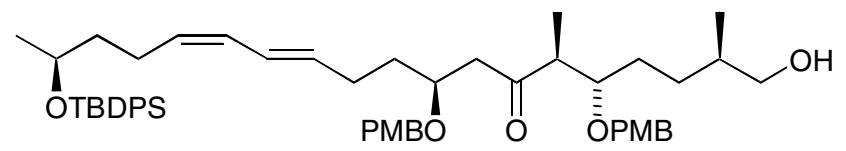

The PMB ether $(570 \mathrm{mg}, 0.59 \mathrm{mmol})$ was desilylated with Dowex $50(4 \mathrm{~g})$ in THF (10 mL) (TP 4) to give after chromatography (hexanes/ ethyl acetate $\left.3: 1, \mathrm{R}_{\mathrm{f}}=0.52\right)(2 \mathrm{R}, 5 \mathrm{~S}, 6 \mathrm{R}, 9 \mathrm{~S}$, 12E, 14Z, 18S) 18- tert-butyl-diphenylsilyloxy 2,6-dimethyl-5,9-di-p-methoxybenzyloxy-1hydroxy-nonadeca-12,14-dien-7-one (450 mg, $89 \%$ ) as a colorless oil.

${ }^{1} \mathrm{H}-\mathrm{NMR}\left(400 \mathrm{MHz} ; \mathrm{CDCl}_{3}\right): \delta[\mathrm{ppm}]=7.69(\mathrm{~d}, \mathrm{~J}=7.4 \mathrm{~Hz}, 4 \mathrm{H}), 7.32-7.44(\mathrm{~m}, 6 \mathrm{H}), 7.22(\mathrm{~d}$, $\mathrm{J}=8.4 \mathrm{~Hz}, 2 \mathrm{H}), 7.18(\mathrm{~d}, \mathrm{~J}=8.4 \mathrm{~Hz}, 2 \mathrm{H}), 6.85(\mathrm{~d}, \mathrm{~J}=8.4 \mathrm{~Hz}, 2 \mathrm{H}), 6.84(\mathrm{~d}, \mathrm{~J}=.8,4 \mathrm{~Hz}, 2 \mathrm{H})$, $6.25(\mathrm{dd}, \mathrm{J}=15.0,10.9 \mathrm{~Hz}, 1 \mathrm{H}), 5.88(\mathrm{dd}, \mathrm{J}=10.9,9.7 \mathrm{~Hz}, 1 \mathrm{H}), 5.58(\mathrm{dt}, \mathrm{J}=15.0,7.0 \mathrm{~Hz}$, $1 \mathrm{H}), 5.19(\mathrm{dt}, \mathrm{J}=9.7,7.4 \mathrm{~Hz}, 1 \mathrm{H}), 4.43(\mathrm{~d}, \mathrm{~J}=10.8 \mathrm{~Hz}, 1 \mathrm{H}), 4.42(\mathrm{~s}, 2 \mathrm{H}), 4.31(\mathrm{~d}, \mathrm{~J}=10.8 \mathrm{~Hz}$, 1H), $3.96(\mathrm{~m}, 1 \mathrm{H}), 3.89$ (qt, J = 6.9, 5.9Hz,1H), 3.79(s, 3H), 3.77(s, 3H) $3.67(\mathrm{~m}, 1 \mathrm{H}), 3.48$ (m, 1H), $3.44(\mathrm{~m}, 1 \mathrm{H}), 2.85(\mathrm{~m}, 2 \mathrm{H}), 2.53(\mathrm{dd}, \mathrm{J}=17.0,5.1 \mathrm{~Hz}, 1 \mathrm{H}), 2.24-2.06(\mathrm{~m}, 4 \mathrm{H}), 1.65-$ $1.36(\mathrm{~m}, 7 \mathrm{H}), 1.27(\mathrm{~m}, 2 \mathrm{H}), 1.08(\mathrm{~d}, \mathrm{~J}=6.9 \mathrm{~Hz}, 3 \mathrm{H}), 1.07(\mathrm{~s}, 9 \mathrm{H}), 1.00(\mathrm{~d}, \mathrm{~J}=6.9 \mathrm{~Hz}, 3 \mathrm{H})$, $0.91(\mathrm{~d}, \mathrm{~J}=6.8 \mathrm{~Hz}, 3 \mathrm{H}) .{ }^{13} \mathrm{C}-\mathrm{NMR}\left(63 \mathrm{MHz} ; \mathrm{CDCl}_{3}\right): \delta[\mathrm{ppm}]=212.4,159.2,159.1,135.9$, 135.8, 134.8, 134.5, 133.8, 130.7, 130.4, 129.9, 129.5, 129.4, 129.3, 128.4, 127.5, 127.4, $125.9,113.7,80.6,74.5,71.7,71.5,69.2,67.9,55.2,49.8,48.6,39.4,35.8,34.4,28.7,27.6$, 27.5, 27.0, 23.6, 23.1, 19.2, 16.7, 12.1. MS : ( FD, 7kV, 3mA) :m/z = 849 [M+1] (29), 848 $[\mathrm{M}](100), 830[\mathrm{M}-16](63) .[\alpha]_{\mathrm{D}}^{20}=+3.4^{\circ}\left(\mathrm{c}=2.0 ; \mathrm{CHCl}_{3}\right)$ IR $($ Film $) 1 / \lambda\left[\mathrm{cm}^{-1}\right]=3468$, 2931, 2858, 1712, 1613, 1587, 1463, 1428, 1376, 1361, 1302, 1249, 1174, 1131, 1111, $1073,1037,998,822,742,704$.

$(2 R, 5 S, 6 R, 9 S, 12 E, 14 Z, 18 S)$-18-tert-Butyldiphenylsilyloxy 2,6-dimethyl-5,9-di-pmethoxybenzyloxy-7-oxo-nonadeca-12,14-dienal (40)

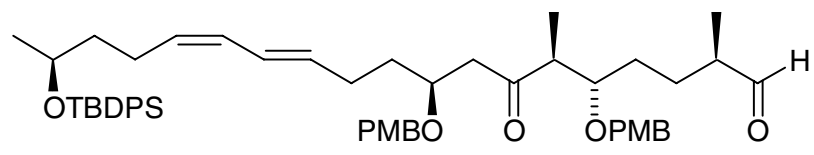

The alcohol obtained above (400 $\mathrm{mg}, 0.47 \mathrm{mmol}$ ) was oxidized under Swern conditions to give 40 (366 mg, $92 \%$ ) after chromatography (hexanes/ ethyl acetate $3: 1\left(\mathrm{R}_{\mathrm{f}}=0.52\right)$ as a 
colorless oil. Anal. Calcd. for $\mathrm{C}_{53} \mathrm{H}_{72} \mathrm{O}_{6} \mathrm{Si}$ : C, 75.14; H, 8.33; found: C, 70.01; H, 8.49. ${ }^{1} \mathrm{H}-$ NMR (400 MHz; $\left.\mathrm{CDCl}_{3}\right): \delta[\mathrm{ppm}]=9 .(\mathrm{d} 7.69(\mathrm{~d}, \mathrm{~J}=7.4 \mathrm{~Hz}, 4 \mathrm{H}), 7.32-7.44(\mathrm{~m}, 6 \mathrm{H}), 7.22$ (d, $\mathrm{J}=8.4 \mathrm{~Hz}, 2 \mathrm{H}), 7.18(\mathrm{~d}, \mathrm{~J}=8.4 \mathrm{~Hz}, 2 \mathrm{H}), 6.85(\mathrm{~d}, 8.4 \mathrm{~Hz}, 2 \mathrm{H}), 6.84(\mathrm{~d}, \mathrm{~J}=.8,4 \mathrm{~Hz}, 2 \mathrm{H}), 6.25$ (dd, $\mathrm{J}=15.0,10.9 \mathrm{~Hz}, 1 \mathrm{H}), 5.88(\mathrm{dd}, \mathrm{J}=10.9,9.7 \mathrm{~Hz}, 1 \mathrm{H}), 5.58$ (dt, J=15.0, 7.0Hz, 1H), 5.19 (dt, $\mathrm{J}=9.7,7.4 \mathrm{~Hz}, 1 \mathrm{H}), 4.43(\mathrm{~d}, \mathrm{~J}=10.8 \mathrm{~Hz}, 1 \mathrm{H}), 4.42(\mathrm{~s}, 2 \mathrm{H}), 4.31$ (d, J=10.8Hz, 1H), 3.96(m, $1 \mathrm{H}), 3.89(\mathrm{qt}, \mathrm{J}=6.9,5.9 \mathrm{~Hz}, 1 \mathrm{H}), 3.79(\mathrm{~s}, 3 \mathrm{H}), 3.77(\mathrm{~s}, 3 \mathrm{H}) 3.67(\mathrm{~m}, 1 \mathrm{H}), 3.48(\mathrm{~m}, 1 \mathrm{H}), 3.44(\mathrm{~m}$, 1H), 2.85 (m, 2H), 2.53 (dd, J=17.0, 5.1Hz, 1H), 2.24-2.06 (m, 4H),1.65-1.36 (m, 7H), 1.27 $(\mathrm{m}, 2 \mathrm{H}), 1.08$ (d, J=6.9Hz, 3H), 1.07 (s, 9H), 1.00 (d, J=6.9Hz, 3H), 0.91 (d, J=6.8Hz, 3H). ${ }^{13} \mathrm{C}-\mathrm{NMR}\left(63 \mathrm{MHz} ; \mathrm{CDCl}_{3}\right): \delta[\mathrm{ppm}]=212.4,159.2,159.1,135.9,135.8,134.8,134.5$, $133.8,130.7,130.4,129.9,129.5,129.4,129.3,128.4,127.5,127.4,125.9,113.7,80.6$, 74.5, 71.7, 71.5, 69.2, 67.9, 55.2, 49.8, 48.6, 39.4, 35.8, 34.4, 28.7,27.6, 27.5, 27.0, 23.6, 23.1, 19.2, 16.7, 12.1. MS : ( FD, 7kV, 3mA) :m/z = 849 [M+1] (29), 848 [M] (100), 830 [M-16] (63). $\alpha^{20}{ }_{\mathrm{D}}=+3.4^{\circ}\left(\mathrm{c}=2 ; \mathrm{CHCl}_{3}\right)$. IR $\left(\right.$ Film) $1 / \lambda\left[\mathrm{cm}^{-1}\right]=2932,2858,1712,1720$, 1613, 1514, 1462, 1428, 1375, 1361, 1302, 1249, 1170, 1136, 1008, 998, 822, 704.

Methyl-(2R/S,3R/S, 4R, 7S, 8R, 11S, 14E, 16Z, 20S)-20-tert-Butyldiphenylsilyloxy-4,8dimethyl-9-oxo-2,7,11-tri-p-methoxybenzyloxy-unicosa-12,14-dienoate

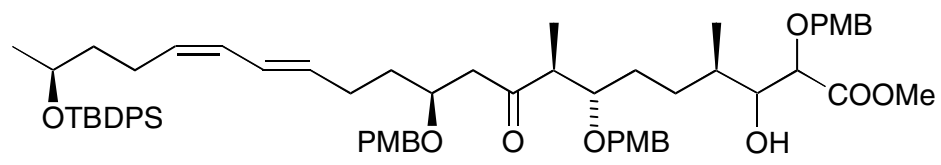

Methyl-O-PMB-glycolate $(8.0 \mathrm{mmol})$ in THF $(16 \mathrm{~mL})$ was treated dropwise at $-90^{\circ} \mathrm{C}$ with 1 M LDA (7 mL) . The mixture was stirred for $15 \mathrm{~min} .40$ (1.5 g, $5.9 \mathrm{mmol})$ in THF ( $10 \mathrm{~mL})$ was added dropwise over $15 \mathrm{~min}$ and the mixture was stirred for $1 \mathrm{~h}$ at $-85^{\circ} \mathrm{C}$. The reaction was quenched with $\mathrm{NH}_{4} \mathrm{Cl}(20 \mathrm{~mL})$. Usual workup furnished after chromatography (hexanes / ethyl acetate $\left.3: 1, \mathrm{R}_{\mathrm{f}}=0.18\right)$ the adduct $(2.1 \mathrm{~g}, 85 \%)$ as a mixture of 4 diastereomers.

Methyl-(2R/S, 4R, 7S, 8R, 11S, 14E, 16Z, 20S)-20-tert-Butyldiphenylsilyloxy-4,8dimethyl-3,9-dioxo-2,7,11-tri-p-methoxybenzyloxy-unikosa-12,14-dienoate (41)

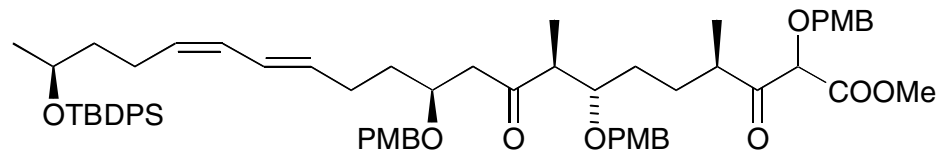

The adduct obtained above ( $240 \mathrm{mg}, 0.23 \mathrm{mmol}$ ) was oxidized under Swern conditions (TP 1) to furnish after chromatography (hexanes/ ethyl acetate $\left.3: 1, \mathrm{R}_{\mathrm{f}}=0.52\right) 41(222 \mathrm{mg}, 93 \%)$ 
as a colorless oil. Anal. Calcd. for $\mathrm{C}_{64} \mathrm{H}_{82} \mathrm{O}_{11} \mathrm{Si}$ : C, 73.55; H, 9.00; found: C, 72.35; H, 9.54. ${ }^{1} \mathrm{H}-\mathrm{NMR}\left(400 \mathrm{MHz} ; \mathrm{CDCl}_{3}\right): \delta[\mathrm{ppm}]=7.66(\mathrm{~m}, 4 \mathrm{H}), 7.32-7.42(\mathrm{~m}, 6 \mathrm{H}), 7.19(\mathrm{dt}$, $\mathrm{J}=8.8,2.0 \mathrm{~Hz}, 1 \mathrm{H}), 7.18(\mathrm{dt}, \mathrm{J}=8.4,2.0 \mathrm{~Hz}, 1 \mathrm{H}), 7.14(\mathrm{dt}, \mathrm{J}=8.8,2.2,1 \mathrm{H}), 7.08(\mathrm{dt}, \mathrm{J}=8.8,2.2$, $1 \mathrm{H}), 6.88(\mathrm{dt}, \mathrm{J}=8.8,2.2,1 \mathrm{H}), 6.86(\mathrm{dt}, \mathrm{J}=8.8,2.2,1 \mathrm{H}), 6.82(\mathrm{dt}, \mathrm{J}=8.4,2.0 \mathrm{~Hz}, 1 \mathrm{H}), 6.79$ (dt, $\mathrm{J}=8.4,2.0,1 \mathrm{H}), 6.78(\mathrm{dt}, \mathrm{J}=8.8,2.0 \mathrm{~Hz}, 1 \mathrm{H}), 6.21(\mathrm{dd}, \mathrm{J}=15.1,11.1 \mathrm{~Hz}, 1 \mathrm{H}), 5.85(\mathrm{dd}$, $\mathrm{J}=11.1,10.9 \mathrm{~Hz}, 1 \mathrm{H}), 5.55(\mathrm{dt}, \mathrm{J}=15.1,6.9 \mathrm{~Hz}, 1 \mathrm{H}), 5.16(\mathrm{dt}, \mathrm{J}=10.9,7.5 \mathrm{~Hz}, 1 \mathrm{H}), 4.84$ (d, $\mathrm{J}=2.2 \mathrm{~Hz}, 0.5 \mathrm{H}), 4.81(\mathrm{~d}, \mathrm{~J}=2.2 \mathrm{~Hz}, 0.5 \mathrm{H}), 4.60(\mathrm{~d}, \mathrm{~J}=5.1 \mathrm{~Hz}, 0.5 \mathrm{H}), 4.57(\mathrm{~d}, \mathrm{~J}=5.1 \mathrm{~Hz}, 0.5 \mathrm{H})$, $4.39(\mathrm{~d}, \mathrm{~J}=1.8 \mathrm{~Hz}, 1 \mathrm{H}), 4.38(\mathrm{~d}, \mathrm{~J}=1.8 \mathrm{~Hz}, 1 \mathrm{H}), 4.36(\mathrm{~d}, \mathrm{~J}=11.0 \mathrm{~Hz}, 0.5 \mathrm{H}), 4.27$ (d, J=5.2Hz, $0.5 \mathrm{H}), 4.24(\mathrm{~d}, \mathrm{~J}=5.2 \mathrm{~Hz}, 0.5 \mathrm{H}), 4.16(\mathrm{~d}, \mathrm{~J}=11.0 \mathrm{~Hz}, 0.5), 3.91(\mathrm{~m}, 1 \mathrm{H}), 3.85(\mathrm{~s}, 1.5 \mathrm{H}), 3.79(\mathrm{~s}$, $1.5 \mathrm{H}), 3.76(\mathrm{~s}, 4.5 \mathrm{H}), 3.75(\mathrm{~s}, 1.5 \mathrm{H}), 3.73(\mathrm{~s}, 3 \mathrm{H}), 3.19(\mathrm{~m}, 1 \mathrm{H}), 2.12(\mathrm{~m}, 4 \mathrm{H}), 1.68-1.86(\mathrm{~m}$, $1 \mathrm{H}), 1.36-1.60(\mathrm{~m}, 7 \mathrm{H}), 1.20(\mathrm{~d}, \mathrm{~J}=6.8 \mathrm{~Hz}, 3 \mathrm{H}), 1.11(\mathrm{~d}, \mathrm{~J}=6.8 \mathrm{~Hz}, 3 \mathrm{H}), 1.05(\mathrm{~d}, \mathrm{~J}=6.8$, $1.5 \mathrm{H}), 1.04(\mathrm{~s}, 9 \mathrm{H}), 0.80(\mathrm{~d}, \mathrm{~J}=6.8 \mathrm{~Hz}, 1.5 \mathrm{H}) .{ }^{13} \mathrm{C}-\mathrm{NMR}\left(63 \mathrm{MHz} ; \mathrm{CDCl}_{3}\right): \delta[\mathrm{ppm}]=212.3$, 202.1, 201.6, 159.1, 135.9, 135.8, 134.8, 134.5, 133.8, 130.4, 130.1, 129.9, 129.5; 129.4, $129.3,128.5,127.5,127.4,125.9,113.7,80.3,74.4,71.5,69.5,69.2,55.2,53.9,48.8,40.4$, $39.9,39.4,34.5 ; 28.7,28.2,27.6,27.4,27.0,23.6,23.1,19.2,18.6,18.4,12.4,12.3$. IR (Film) $1 / \lambda\left[\mathrm{cm}^{-1}\right]=2934 ; 1729,1514,1250,1036$.

\section{Methyl-(2R/S, 4R, 7S, 8R, 11S, 14E, 16Z, 20S)-4,8-Dimethyl-3,9-dioxo-20-hydroxy-} 2,7,11-tri-p-methoxybenzyloxy-unicosa-12,14-dienoate (42)

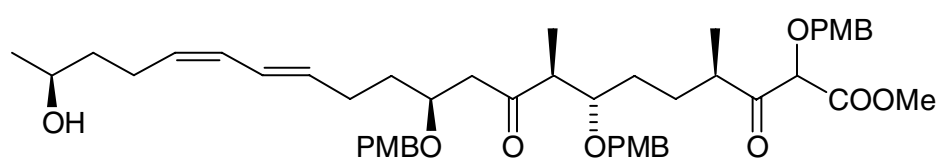

41 (200 mg, $0.19 \mathrm{mmol}$ ) was desilylated with HF in acetonitrile (TP 5) to give after chromatography (hexanes/ ethyl acetate $3: 1) 42(101 \mathrm{mg}, 65 \%)$ as a colorless oil. Anal. calcd for $\mathrm{C}_{48} \mathrm{H}_{64} \mathrm{O}_{11}$ : C, 70.56; H, 7.90; found: C, 70.40, H, 7.71. ${ }^{1} \mathrm{H}-\mathrm{NMR}$ (400 MHz; $\left.\mathrm{CDCl}_{3}\right): \delta[\mathrm{ppm}]=7.19(\mathrm{dt}, \mathrm{J}=8.8,2.0 \mathrm{~Hz}, 1 \mathrm{H}), 7.18(\mathrm{dt}, \mathrm{J}=8.4,2.0 \mathrm{~Hz}, 1 \mathrm{H}), 7.14(\mathrm{dt}, \mathrm{J}=8.8$, 2.2, 1H), 7.08 (dt, J=8.8, 2.2, 1H), $6.88(\mathrm{dt}, \mathrm{J}=8.8,2.2,1 \mathrm{H}), 6.86(\mathrm{dt}, \mathrm{J}=8.8,2.2,1 \mathrm{H}), 6.82$ $(\mathrm{dt}, \mathrm{J}=8.4,2.0 \mathrm{~Hz}, 1 \mathrm{H}), 6.79(\mathrm{dt}, \mathrm{J}=8.4,2.0,1 \mathrm{H}), 6.78(\mathrm{dt}, \mathrm{J}=8.8,2.0 \mathrm{~Hz}, 1 \mathrm{H}), 6.21(\mathrm{dd}$, $\mathrm{J}=15.1,11.1 \mathrm{~Hz}, 1 \mathrm{H}), 5.85(\mathrm{dd}, \mathrm{J}=11.1,10.9 \mathrm{~Hz}, 1 \mathrm{H}), 5.55$ (dt, J=15.1, 6.9Hz, 1H), 5.16 (dt, $\mathrm{J}=10.9,7.5 \mathrm{~Hz}, 1 \mathrm{H}), 4.84(\mathrm{~d}, \mathrm{~J}=2.2 \mathrm{~Hz}, 0.5 \mathrm{H}), 4.81(\mathrm{~d}, \mathrm{~J}=2.2 \mathrm{~Hz}, 0.5 \mathrm{H}), 4.60(\mathrm{~d}, \mathrm{~J}=5.1 \mathrm{~Hz}$, $0.5 \mathrm{H}), 4.57(\mathrm{~d}, \mathrm{~J}=5.1 \mathrm{~Hz}, 0.5 \mathrm{H}), 4.39$ (d, J=1.8Hz, 1H), 4.38 (d, J=1.8Hz, 1H), 4.36 (d, 
$\mathrm{J}=11.0 \mathrm{~Hz}, 0.5 \mathrm{H}), 4.27(\mathrm{~d}, \mathrm{~J}=5.2 \mathrm{~Hz}, 0.5 \mathrm{H}), 4.24(\mathrm{~d}, \mathrm{~J}=5.2 \mathrm{~Hz}, 0.5 \mathrm{H}), 4.16(\mathrm{~d}, \mathrm{~J}=11.0 \mathrm{~Hz}, 0.5)$, $3.91(\mathrm{~m}, 1 \mathrm{H}), 3.85(\mathrm{~s}, 1.5 \mathrm{H}), 3.79(\mathrm{~s}, 1.5 \mathrm{H}), 3.76(\mathrm{~s}, 4.5 \mathrm{H}), 3.75(\mathrm{~s}, 1.5 \mathrm{H}), 3.73(\mathrm{~s}, 3 \mathrm{H}), 3.19$ (m, 1H), $2.12(\mathrm{~m}, 4 \mathrm{H}), 1.68-1.86(\mathrm{~m}, 1 \mathrm{H}), 1.36-1.60(\mathrm{~m}, 7 \mathrm{H}), 1.20(\mathrm{~d}, \mathrm{~J}=6.8 \mathrm{~Hz}, 3 \mathrm{H}), 1.11$ $(\mathrm{d}, \mathrm{J}=6.8 \mathrm{~Hz}, 3 \mathrm{H}), 1.05(\mathrm{~d}, \mathrm{~J}=6.8,1.5 \mathrm{H}), 0.80(\mathrm{~d}, \mathrm{~J}=6.8 \mathrm{~Hz}, 1.5 \mathrm{H}) .{ }^{13} \mathrm{C}-\mathrm{NMR}(63 \mathrm{MHz}$; $\left.\mathrm{CDCl}_{3}\right): \delta[\mathrm{ppm}]=212.3,202.1,201.6,159.1,135.9,135.8,134.8,134.5,133.8,130.4$, $130.1,129.9,129.5 ; 129.4,129.3,128.5,127.5,127.4,125.9,113.7,80.3$, 74.4, 71.5, 69.5, 69.2, 55.2, 53.9, 48.8, 40.4, 39.9, 39.4, 34.5; 28.7, 28.2, 27.6, 27.4, 27.0, 23.6, 23.1, 19.2, $18.6,18.4,12.4,12.3$.

(4R)-5-tert-Butyldimethysilyloxy-4-methyl-pentanal (46)

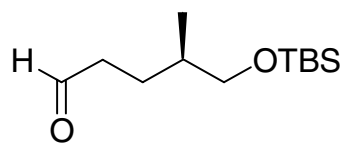

45 (15.7 g, $66.7 \mathrm{mmol})$ was reduced with DIBAL-H (TP 2) to give, after distillation $\left(68^{\circ} \mathrm{C}\right.$, $0.5 \mathrm{~mm} \mathrm{Hg}) 46$ (12.3 g, 89\%). Anal. calcd. for $\mathrm{C}_{12} \mathrm{H}_{26} \mathrm{O}_{2} \mathrm{Si}: \mathrm{C}, 62.55 ; \mathrm{H}, 11.37$; found: $\mathrm{C}$, 62.39; H, 11.20. ${ }^{1} \mathrm{H}-\mathrm{NMR}\left(400 \mathrm{MHz} ; \mathrm{CDCl}_{3}\right): \delta[\mathrm{ppm}]=9.98(\mathrm{t}, J=2.0 \mathrm{~Hz}, 1 \mathrm{H}), 3.43(\mathrm{~d}$, $5.7 \mathrm{~Hz}, 1 \mathrm{H}), 3.42$ (d, $J=5.9 \mathrm{~Hz}, 1 \mathrm{H}), 2.47$ (dddd, $J=6.9,4.8,2.5,2.0 \mathrm{~Hz}, 1 \mathrm{H}$ ), 2.43 (dddd, $\mathrm{J}=6.9,5.0,3.2,2.0 \mathrm{~Hz}, 1 \mathrm{H}), 1.75(\mathrm{~m}, 1 \mathrm{H}), 1.61(\mathrm{~m}, 1 \mathrm{H}), 1.47(\mathrm{~m}, 1 \mathrm{H}), 0.89(\mathrm{~s}, 9 \mathrm{H}), 0.88(\mathrm{~d}$, $J=6.6 \mathrm{~Hz}, 3 \mathrm{H}), 0.04(\mathrm{~s} ; 6 \mathrm{H}) ;{ }^{13} \mathrm{C}-\mathrm{NMR}\left(63 \mathrm{MHz} ; \mathrm{CDCl}_{3}\right): \delta[\mathrm{ppm}]=202.8,67.8,41.6,35.2$, 25.8, 25.5, 18.3, 16.5, -5.5; MS: (EI, $\left.70 \mathrm{eV}, 90^{\circ} \mathrm{C}\right) \mathrm{m} / \mathrm{z}=215$ [M-15](2), 189 (4), 173 [M57] (36), 171 (14), 155 (8), 131 (10), 105 (9), 99 (11), 81 (13), 75 (100); HRMS (EI) calcd for $\mathrm{C}_{8} \mathrm{H}_{17} \mathrm{O}_{2} \mathrm{Si}\left[\mathrm{M}-\mathrm{C}_{4} \mathrm{H}_{9}\right]: 173.0998$ found : 173.1003; $[\alpha]_{\mathrm{D}}^{20}=+10.0^{\circ}\left(\mathrm{c}=2.0 ; \mathrm{CH}_{2} \mathrm{Cl}_{2}\right)$; IR (thin film) $1 / \lambda\left[\mathrm{cm}^{-1}\right]=2957,2930,2886,2858,1728,1472,1255,1006,837,776$.

$(2 R, 5 S, 6 R)$-1-tert-Butyldimethylsilyloxy-2,6-dimethyloct-7-en-5-ol (47)

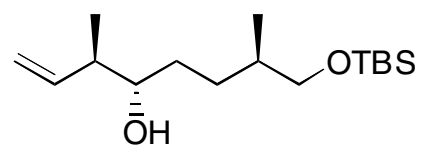

Crotylmagnesium chloride $\left(5 \mathrm{~mL}, 5 \mathrm{mmol}, 1 \mathrm{M}\right.$ in ether) was added dropwise at $0^{\circ} \mathrm{C}$ to a solution of (4S,5S)-cyclopentadienyl[(4,5 trans)-2,2-dimethyl- $\alpha, \alpha, \alpha$ ', $\alpha^{\prime}$-tetraphenyl-1,3dioxolane-4,5-dimethanolato-O,O']titanium chloride (3.68g, $6 \mathrm{mmol})$ in $50 \mathrm{~mL}$ of ether over $10 \mathrm{~min}$. After stirring for $1 \mathrm{~h}$ at $0^{\circ} \mathrm{C}$, the orange suspension was cooled to $-78^{\circ} \mathrm{C}$ and $46(1.15$ 
g, $5 \mathrm{mmol}$ ) in $10 \mathrm{~mL} \mathrm{Et}_{2} \mathrm{O}$ was added over $15 \mathrm{~min}$. Stirring at $-78^{\circ} \mathrm{C}$ was continued for $3 \mathrm{~h}$. The reaction mixture was then treated with $10 \mathrm{~mL}$ of aqueous $45 \% \mathrm{NH}_{4} \mathrm{~F}$ solution, vigorously stirred for $12 \mathrm{~h}$, filtered through Celite and extracted with $\mathrm{Et}_{2} \mathrm{O}(3 \times 30 \mathrm{~mL})$. The combined organic phases were washed with brine, dried over $\mathrm{MgSO}_{4}$ and concentrated. The residue was stirred with $30 \mathrm{~mL}$ of pentane. Subsequent filtration furnished $1.1 \mathrm{~g}$ of recovered ligand. Chromatography $\left(\mathrm{SiO}_{2}, 200 \mathrm{~g}\right.$, hexane/EtOAc $\left.5: 1, \mathrm{R}_{\mathrm{f}}=0.48\right)$ of the residue finally afforded $1.11 \mathrm{~g}(81 \%)$ of 47 as a colorless oil. Anal. calcd for $\mathrm{C}_{16} \mathrm{H}_{34} \mathrm{O}_{2} \mathrm{Si}$ : C 67.07; H, 11.96; found: C, 67.30; H, 11.73; ${ }^{1} \mathrm{H}-\mathrm{NMR}\left(400 \mathrm{MHz} ; \mathrm{CDCl}_{3}\right): \delta[\mathrm{ppm}]=5.77(\mathrm{ddd}, J=17.2,10.4,8.2 \mathrm{~Hz}$, 1H), 5.13 (ddd, $J=10.4,1.9,1.0 \mathrm{~Hz}, 1 \mathrm{H}), 5.12$ (ddd, $J=17.2,1.9,0.8 \mathrm{~Hz}, 1 \mathrm{H}), 3.46$ (dd, $J$ $=9.8,5.5 \mathrm{~Hz}, 1 \mathrm{H}), 3.40(\mathrm{~m}, 1 \mathrm{H}), 3.39(\mathrm{dd}, J=9.8,6.2 \mathrm{~Hz}, 1 \mathrm{H}), 2.23$ (qtdd, $J=6.9,6.5,1.0$, $0.8 \mathrm{~Hz}, 1 \mathrm{H}), 1.59$ (m, 4H), 1.36 (m, 1H), 1.13 (m, 1H), 1.04 (d, J=6.9 Hz, 1H), 0.9 (s, 9H), $0.89(\mathrm{~d}, J=5.0 \mathrm{~Hz}, 3 \mathrm{H}), 0.04(\mathrm{~s}, 6 \mathrm{H}) ;{ }^{13} \mathrm{C}-\mathrm{NMR}\left(63 \mathrm{MHz} ; \mathrm{CDCl}_{3}\right): \delta[\mathrm{ppm}]=140.3,115.2$, $75.2,68.3,43.9,36.0,31.8,29.0,26.0,18.4,17.0,16.4,-5.3 ; \mathrm{MS}:\left(\mathrm{EI}, 70 \mathrm{eV}, 90^{\circ} \mathrm{C}\right) \mathrm{m} / \mathrm{z}$ $=237[\mathrm{M}-59]$ (4), 207 (14), 183 (21), 137 (15), 105 (50), 99 (41), 95 (39), $81(100)$; $[\alpha]_{\mathrm{D}}^{20}=$ $+7.4^{\circ}\left(\mathrm{c}=2.0 ; \mathrm{CH}_{2} \mathrm{Cl}_{2}\right)$; IR (thin film) $1 / \lambda\left[\mathrm{cm}^{-1}\right]=3422,3385,2957,2932,2886,2858$, 1462, 1255, 966, 912, 837, 775, 667.

$(2 R, 5 S, 6 R)$-1-tert-Butyldimethylsilyloxy-2,6-dimethyl-5-tetrahydropyranyloxyoct-7-ene

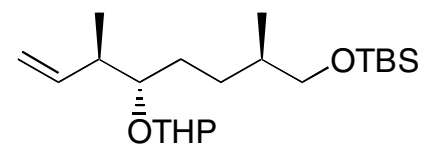

$(2 R, 5 S, 6 R)$-1-tert-Butyldimethylsilyloxy-2,6-dimethyloct-7-en-5-ol 47 (7.0 g, 24.4 mmol) and camphorsulfonic acid $(25 \mathrm{mg}, 0.11 \mathrm{mmol})$ were dissolved in $30 \mathrm{~mL}$ of $\mathrm{CH}_{2} \mathrm{Cl}_{2}$. 3,4Dihydro-2H-pyran $(2.9 \mathrm{~mL}, 31.8 \mathrm{mmol})$ was added dropwise and the solution was stirred for $12 \mathrm{~h}$. The reaction was quenched with $10 \mathrm{~mL}$ of saturated aqueous $\mathrm{NaHCO}_{3}$ and phases were separated. The aqueous phase was extracted three times with $\mathrm{CH}_{2} \mathrm{Cl}_{2}$, the combined organic layers were washed with brine, dried over $\mathrm{MgSO}_{4}$, filtered and concentrated in vacuo. Chromatography $\left(\mathrm{SiO}_{2}, 400 \mathrm{~g}\right.$, hexane/EtOAc, $\left.10: 1, \mathrm{R}_{\mathrm{f}}=0.71\right)$ of the residue afforded 8.69 g $(96 \%) \quad$ of $\quad(2 R, \quad 5 S, \quad 6 R)$-1-tert-butyldimethylsilyloxy-2,6-dimethyl-5tetrahydropyranyloxyoct-7-ene as a colorless oil. Anal. calcd for $\mathrm{C}_{21} \mathrm{H}_{42} \mathrm{O}_{3} \mathrm{Si}: \mathrm{C}, 68.05 ; \mathrm{H}$, 11.42. found: C, 67.93; $\mathrm{H}, 11.17$. ${ }^{1} \mathrm{H}-\mathrm{NMR}\left(250 \mathrm{MHz} ; \mathrm{CDCl}_{3}\right): \delta[\mathrm{ppm}]=5.85(\mathrm{ddd}, J=$ 
14.9, 10.7, 7.3 Hz, 0.5H), 5.78 (ddd, $J=15.1,10.1,7.3 \mathrm{~Hz}, 0.5 \mathrm{H}), 5.02$ (dt, $J=15.1,1.0 \mathrm{~Hz}$, $0.5 \mathrm{H}), 5.02(\mathrm{dt}, J=14.9,1.0 \mathrm{~Hz}, 0.5 \mathrm{H}), 5.01(\mathrm{dt}, J=10.7,1.0 \mathrm{~Hz}, 0.5 \mathrm{H}), 5.01(\mathrm{dt}, J=10.1$, $1.0 \mathrm{~Hz}, 0.5 \mathrm{H}), 4.65(\mathrm{~m}, 1 \mathrm{H}), 3.93(\mathrm{~m}, 1 \mathrm{H}), 3.31-3.53(\mathrm{~m}, 4 \mathrm{H}), 2.48(\mathrm{~m}, 1 \mathrm{H}), 1.83(\mathrm{~m}, 1 \mathrm{H})$, $1.39-1.76(\mathrm{~m}, 10 \mathrm{H}), 1.06(\mathrm{~d}, J=6.9 \mathrm{~Hz}, 1.5 \mathrm{H}), 0.99$ (d, $J=6.9 \mathrm{~Hz}, 1.5 \mathrm{H}), 0.89$ (s, 9H), 0.87 $(\mathrm{d}, J=6.6 \mathrm{~Hz}, 1.5 \mathrm{H}), 0.85(\mathrm{~d}, J=6.6 \mathrm{~Hz}, 1.5 \mathrm{H}), 0.04(\mathrm{~s}, 6 \mathrm{H}) ;{ }^{13} \mathrm{C}-\mathrm{NMR}\left(63 \mathrm{MHz} ; \mathrm{CDCl}_{3}\right): \delta$ $[\mathrm{ppm}]=141.2,140.8,114.5,114.2,98.3,98.2,81.1,80.9,68.4,68.3,62.6,62.5,41.5,40.0$, 36.0, 31.2, 31.0, 29.3, 29.1, 29.0, 27.6, 26.0, 25.6, 19.9, 19.8, 16.8, 16.7, 15.3, 14.8, -5.4; MS: (EI, 70eV, 90 $\mathrm{C}) \mathrm{m} / \mathrm{z}=370$ [M] (0.3), 313 [M-57] (4), 269 (35), 231 (80), 201 (14), $173(83), 159(97), 137(35), 115(21), 101(16), 95(63), 85(100) ;[\alpha]_{\mathrm{D}}^{20}=+9.9$ (c =2.0; $\mathrm{CH}_{2} \mathrm{Cl}_{2}$ ); IR (thin film): $1 / \lambda\left[\mathrm{cm}^{-1}\right]=2954,2857,1472,1463,1256,1133,1115,1096$, $1078,1032,1024,1007,911,837,775$.

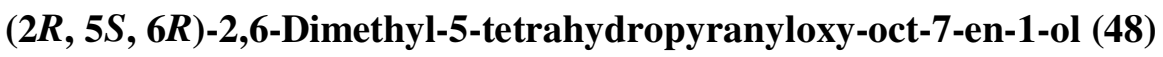

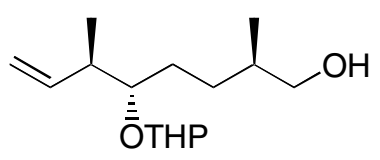

A stock solution of $5 \mathrm{~mL} \mathrm{HF} /$ pyridine complex, $10 \mathrm{~mL}$ pyridine and $15 \mathrm{~mL}$ THF was prepared. In a teflon vessel $(2 R, 5 S, 6 R)$-1-tert-butyldimethylsilyloxy-2,6-dimethyl-5tetrahydropyranyloxy-oct-7-ene $(2.35 \mathrm{~g}, 6.34 \mathrm{mmol})$ was dissolved in $10 \mathrm{~mL}$ THF and $5 \mathrm{~mL}$ of the stock solution was added slowly at ambient temperature. The mixture was stirred for 2 $\mathrm{h}$ and then diluted with $\mathrm{Et}_{2} \mathrm{O}(30 \mathrm{~mL})$ and saturated aqueous $\mathrm{CuSO}_{4}(15 \mathrm{~mL})$. The phases were separated and the aqueous layer was extracted three times with $\mathrm{Et}_{2} \mathrm{O}$. The combined organic layers were washed with saturated aqueous $\mathrm{NaHCO}_{3}$, dried over $\mathrm{MgSO}_{4}$ and concentrated in vacuo. The residue was purified by column chromatography $\left(\mathrm{SiO}_{2}, 150 \mathrm{~g}\right.$, EtOAc/hexanes $\left.1: 1, \mathrm{R}_{\mathrm{f}}=0.51\right)$ to provide $48(1.58 \mathrm{~g}, 97 \%)$ as a clear colorless oil. Anal. calcd for $\mathrm{C}_{15} \mathrm{H}_{28} \mathrm{O}_{3}$ : C, 70.27; H, 11.01; found: C, 70.24; H, 10.96; ${ }^{1} \mathrm{H}-\mathrm{NMR}(400 \mathrm{MHz}$; $\left.\mathrm{CDCl}_{3}\right): \delta[\mathrm{ppm}]=5.83(\mathrm{ddd}, J=17.1,10.5,7.1 \mathrm{~Hz}, 0.5 \mathrm{H}), 5.77(\mathrm{ddd}, J=17.6,10.0,7.5$ $\mathrm{Hz}, 0.5 \mathrm{H}), 5.02(\mathrm{~d}, J=17.7 \mathrm{~Hz}, 0.5 \mathrm{H}), 5.02(\mathrm{~d}, J=17.1 \mathrm{~Hz}, 0.5 \mathrm{H}), 5.01(\mathrm{dt}, J=10.5,1.0$ $\mathrm{Hz}, 0.5 \mathrm{H}), 5.01(\mathrm{dt}, J=10.0,1.0 \mathrm{~Hz}, 0.5 \mathrm{H}), 4.65(\mathrm{dd}, J=4.5,2.5 \mathrm{~Hz}, 0.5 \mathrm{H}), 4.59(\mathrm{dd}, J=$ 5.0, $2.5 \mathrm{~Hz}, 0.5 \mathrm{H}), 3.93(\mathrm{~m}, 1 \mathrm{H}), 3.31-3.53(\mathrm{~m}, 4 \mathrm{H}), 2.52(\mathrm{qt}, J=6.5,6.3 \mathrm{~Hz}, 0.5 \mathrm{H}), 2.42$ (qt, $J=7.0,5.9 \mathrm{~Hz}, 0.5 \mathrm{H}), 1.81(\mathrm{~m}, 1 \mathrm{H}), 1.39-1.76(\mathrm{~m}, 10 \mathrm{H}), 1.17(\mathrm{~m}, 1 \mathrm{H}), 1.07(\mathrm{~d}, J=7.0$ $\mathrm{Hz}, 1.5 \mathrm{H}), 0.98(\mathrm{~d}, J=6.5 \mathrm{~Hz}, 1.5 \mathrm{H}), 0.90(\mathrm{~d}, J=6.5 \mathrm{~Hz}, 3 \mathrm{H}) ;{ }^{13} \mathrm{C}-\mathrm{NMR}\left(63 \mathrm{MHz} ; \mathrm{CDCl}_{3}\right)$ : 
$\delta[\mathrm{ppm}]=141.1,140.7$,114.6, 114.3, 99.1, 98.3, 81.4, 81.0, 68.2, 67.9, 63.4, 62.7, 41.4, 40.4, 36.0, 35.9, 31.2, 31.1, 29.1, 29.0, 28.8, 28.6, 25.6, 25.5, 20.3, 19.8, 16.7, 16.6, 15.3, 14.8; MS (EI, 70eV, 60 C); m/z = 183 [M-73] (5), 171 (2), 155 (8), 137 (8), 117 (7), 99 (56), 95 (19), $86(39), 85$ (100), $81(49) ;[\alpha]_{\mathrm{D}}^{20}=+17.5^{\circ}\left(\mathrm{c}=2.0 ; \mathrm{CH}_{2} \mathrm{Cl}_{2}\right) ; \mathrm{IR}$ (thin film): $1 / \lambda$ $\left[\mathrm{cm}^{-1}\right]=3420,2943,2872,1455,1441,1379,1352,1200,1133,1115,1077,1024,911$.

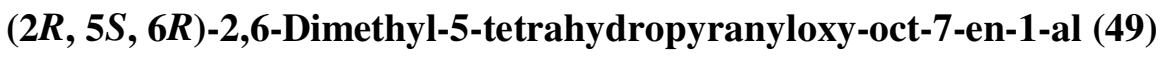

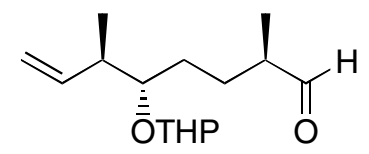

48 (2.4 g, $9.4 \mathrm{mmol}$ ) was oxidized under Swern conditions (TP 1) to give, after column chromatography (200 $\mathrm{g}$ of $\mathrm{SiO}_{2}$, hexanes/ EtOAc $\left.3: 1, \mathrm{R}_{\mathrm{f}}=0.52\right), 49(2.3 \mathrm{~g}, 97 \%)$ as a colorless oil as a mixture of 2 diastereomers: Anal. calcd for $\mathrm{C}_{15} \mathrm{H}_{26} \mathrm{O}_{3}: \mathrm{C} 70.83 ; \mathrm{H}, 10.30$; found: C, 70.61; H, 10.09; ${ }^{1} \mathrm{H}-\mathrm{NMR}\left(250 \mathrm{MHz}, \mathrm{CDCl}_{3}\right): \delta[\mathrm{ppm}]=9.61(\mathrm{~d}, J=2.1 \mathrm{~Hz}$, $0.5 \mathrm{H}), 9.60(\mathrm{~d}, J=2.1 \mathrm{~Hz}, 0.5 \mathrm{H}), 5.83(\mathrm{ddd}, J=15.3,9.9,7.3 \mathrm{~Hz}, 0.5 \mathrm{H}), 5.75$ (ddd, $J=$ $15.1,9.9,7.1 \mathrm{~Hz}, 0.5 \mathrm{H}), 5.03(\mathrm{dt}, J=15.3,1.0 \mathrm{~Hz}, 0.5 \mathrm{H}), 5.03(\mathrm{dt}, J=15.1,1.0 \mathrm{~Hz}, 0.5 \mathrm{H})$, $5.02(\mathrm{dt}, J=9.9,1.0 \mathrm{~Hz}, 0.5 \mathrm{H}), 5.01(\mathrm{dt}, J=9.9,1.0 \mathrm{~Hz}, 0.5 \mathrm{H}), 4.58-4.66(\mathrm{~m}, 1 \mathrm{H}), 3.93(\mathrm{~m}$, $1 \mathrm{H}), 3.41-3.53(\mathrm{~m}, 2 \mathrm{H}), 2.25-2.58(\mathrm{~m}, 2 \mathrm{H}), 1.34-1.95(\mathrm{~m}, 10 \mathrm{H}), 1.08(\mathrm{~d}, J=7.1 \mathrm{~Hz}, 3 \mathrm{H})$, $1.06(\mathrm{~d}, J=6.5 \mathrm{~Hz}, 1.5 \mathrm{H}), 0.98(\mathrm{~d}, J=7.1 \mathrm{~Hz}, 1.5 \mathrm{H}) ;{ }^{13} \mathrm{C}-\mathrm{NMR}\left(63 \mathrm{MHz} ; \mathrm{CDCl}_{3}\right): \delta[\mathrm{ppm}]$ $=205.3,204.9,140.9,140.5,114.8,114.5,98.8,98.5,80.7,80.5,63.3,62.7,46.4,46.3$, 41.3, 31.2, 31.1, 28.8, 27.4, 26.8, 25.5, 25.4, 20.3, 15.0, 14.5, 13.4; MS: (FI, 7kV, 3mA, $\left.20^{\circ} \mathrm{C}\right) \mathrm{m} / \mathrm{z}=255[\mathrm{M}+1](3), 199(93), 153(6), 85(100) ;[\alpha]_{\mathrm{D}}^{20}=-9.2^{\circ}\left(\mathrm{c}=2.0 ; \mathrm{CH}_{2} \mathrm{Cl}_{2}\right), \mathrm{IR}$ (thin film) $1 / \lambda\left[\mathrm{cm}^{-1}\right]=2942,2871,1727,1455,1442,1376,1352,1200,1160,1132,1115$, $1077,1034,912,870$.

Methyl $(2 R / S, 3 R / S, 4 R, 7 S, 8 R)-4,8$-dimethyl-2,5-ditetrahydropyranyloxy-3-hydroxydec-9-enoate (50)

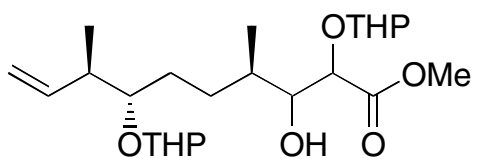


Methyl 2-tetrahydropyranyloxy-acetate $(1.4 \mathrm{~g}, 8.0 \mathrm{mmol})$ in $16 \mathrm{~mL}$ THF was cooled to $90^{\circ} \mathrm{C}$. A freshly prepared $1 \mathrm{M} \mathrm{LDA}$-solution $(7 \mathrm{~mL}, 7 \mathrm{mmol})$ was added and mixture was stirred for a further $20 \mathrm{~min} .49(1.5 \mathrm{~g}, 5.9 \mathrm{mmol})$ in $10 \mathrm{~mL}$ THF was added over a period of $15 \mathrm{~min}$ and stirring was continued for $30 \mathrm{~min}$ at $-85^{\circ} \mathrm{C}$. The reaction was quenched with 20 $\mathrm{mL}$ of a saturated aqueous $\mathrm{NH}_{4} \mathrm{Cl}$ and phases were separated. The aqueous phase was extracted three times with $\mathrm{Et}_{2} \mathrm{O}$, the combined organic layers were washed with brine, dried over $\mathrm{MgSO}_{4}$, filtered and concentrated in vacuo. The residue was purified by column chromatography $\left(300 \mathrm{~g}\right.$ of $\mathrm{SiO}_{2}$, EtOAc/hexanes $\left.1: 1, \mathrm{Rf}=0.52\right)$ to provide $\mathbf{5 0}(2.1 \mathrm{~g}, 83 \%)$ as a mixture of 16 diastereomers: Anal. calcd for $\mathrm{C}_{23} \mathrm{H}_{40} \mathrm{O}_{7}: \mathrm{C}, 64.46 ; \mathrm{H}, 9.41$; found: $\mathrm{C}$, 64.17; H, 9.12; ${ }^{1} \mathrm{H}-\mathrm{NMR}\left(250 \mathrm{MHz} ; \mathrm{CDCl}_{3}\right): \delta[\mathrm{ppm}]=5.70-5.96(\mathrm{~m}, 1 \mathrm{H}), 5.02-5.10(\mathrm{~m}$, 2H), 4.62-4.80 (m, 2H), 3.70-4.16 (m, 4H), $3.80(\mathrm{~s}, 3 \mathrm{H}), 3.42-3.56(\mathrm{~m}, 3 \mathrm{H}), 2.38-2.58(\mathrm{~m}$, 2H), 1.56-1.94 (m, 17H), $1.07(\mathrm{~d}, J=6.9 \mathrm{~Hz}, 1.5 \mathrm{H}), 0.99(\mathrm{~d}, J=7.1 \mathrm{~Hz}, 1.5 \mathrm{H}), 0.95(\mathrm{~d}, J=$ $6.9 \mathrm{~Hz}, 1.5 \mathrm{H}), 0.90(\mathrm{~d}, J=6.5 \mathrm{~Hz}, 1.5 \mathrm{H}) ; \mathrm{MS}:\left(\mathrm{FI}, 7 \mathrm{kV}, 3 \mathrm{~mA}, 155^{\circ} \mathrm{C}\right) \mathrm{m} / \mathrm{z}=429[\mathrm{M}+1](3)$, 187 (68), 141 (100); IR (thin film) $1 / \lambda\left[\mathrm{cm}^{-1}\right]=3450,2948,2871,1748,1455,1440,1383$, $1352,1200,1184,1132,1122,1077,1036,1025,912,870$.

\section{Methyl (2R/S, 4R, 7S, 8R)-4,8-dimethyl-2,5-ditetrahydropyranyloxy-3-oxo-dec-9-} enoate (51)

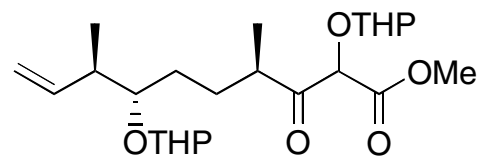

50 was oxidized under Swern conditions (TP 1) to give after column chromatography (20g of $\mathrm{SiO}_{2}$, hexane/EtOAc $\left.3: 1, \mathrm{R}_{\mathrm{f}}=0.39\right) \mathbf{5 1}(1.53 \mathrm{~g}, 75 \%)$ as a colorless oil as a mixture of 8 diastereomers: Anal. calcd for $\mathrm{C}_{23} \mathrm{H}_{38} \mathrm{O}_{7}$ : C, 64.76; H, 8.98; found: C, 64.91; H, 9.12; ${ }^{1} \mathrm{H}$ NMR (250 MHz; $\left.\mathrm{CDCl}_{3}\right): \delta[\mathrm{ppm}]=5.68-5.90(\mathrm{~m}, 1 \mathrm{H}), 5.02(\mathrm{~d}, J=16.0 \mathrm{~Hz}, 1 \mathrm{H}), 5.01(\mathrm{~d}, J$ $=11.4 \mathrm{~Hz}, 1 \mathrm{H}), 4.93(\mathrm{~s}, 0.125 \mathrm{H}), 4.92(\mathrm{~s}, 0.125 \mathrm{H}), 4.91(\mathrm{~s}, 0.125 \mathrm{H}), 4.89(\mathrm{~s}, 0.125 \mathrm{H}), 4.82$ $(\mathrm{s}, 0.125 \mathrm{H}), 4.80(\mathrm{~s}, 0.125 \mathrm{H}), 4.76(\mathrm{~s}, 0.125 \mathrm{H}), 4.73(\mathrm{~s}, 0.125 \mathrm{H}), 4.69-4.78(\mathrm{~m}, 1 \mathrm{H}), 4.58-$ $4.66(\mathrm{~m}, 0.5 \mathrm{H}), 4.50-4.56(\mathrm{~m}, 0.5 \mathrm{H}), 3.78(\mathrm{~s}, 3 \mathrm{H}), 3.70-3.92(\mathrm{~m}, 3 \mathrm{H}), 3.40-3.60(\mathrm{~m}, 2 \mathrm{H})$, 2.80-3.10 (m, 2H), $2.20(\mathrm{~s}, 1.5 \mathrm{H}), 2.16(\mathrm{~s}, 1.5 \mathrm{H}), 1.70-1.85(\mathrm{~m}, 4 \mathrm{H}), 1.40-1.70(\mathrm{~m}, 9 \mathrm{H})$, $1.20-1.35(\mathrm{~m}, 1 \mathrm{H}), 1.15(\mathrm{~d}, J=7.1 \mathrm{~Hz}, 0.375 \mathrm{H}), 1.14(\mathrm{~d}, J=6.9 \mathrm{~Hz}, 0.375 \mathrm{H}), 1.13(\mathrm{~d}, J=$ $7.1 \mathrm{~Hz}, 0.375 \mathrm{H}), 1.12(\mathrm{~d}, J=7.1 \mathrm{~Hz}, 0.375 \mathrm{H}), 1.11(\mathrm{~d}, J=7.1 \mathrm{~Hz}, 0.75 \mathrm{H}), 1.09(\mathrm{~d}, J=7.1$ 
$\mathrm{Hz}, 0.375 \mathrm{H}), 1.08(\mathrm{~d}, J=7.1 \mathrm{~Hz}, 0.375 \mathrm{H}), 1.04(\mathrm{~d}, J=7.1 \mathrm{~Hz}, 0.75 \mathrm{H}), 1.03(\mathrm{~d}, J=7.1 \mathrm{~Hz}$, $0.375 \mathrm{H}), 0.98(\mathrm{~d}, J=7.1 \mathrm{~Hz}, 0.75 \mathrm{H}), 0.97(\mathrm{~d}, J=7.1 \mathrm{~Hz}, 0.75 \mathrm{H}), 0.87(\mathrm{~d}, J=7.1 \mathrm{~Hz}$, 0.375H); MS: (FI, 7kV, 3mA, 95 $\left.{ }^{\circ} \mathrm{C}\right) \mathrm{m} / \mathrm{z}=426$ [M] (12), 325 (17), 253 (16), 173 (27), 113 (100); IR (thin film) $1 / \lambda\left[\mathrm{cm}^{-1}\right]=2942,2873,1751,1723,1455,1380,1353,1261,1201$, $1185,1132,1078,1036,1024,965,911,870$.

\section{Methyl $(2 R / S, 4 R, 7 S, 8 R)-4,8$-dimethyl-2,5-ditetrahydropyranyloxy-3,9-dioxodecanoate} (52)

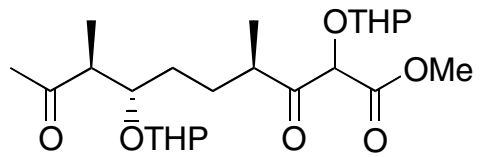

To a solution of $51(3.2 \mathrm{~g}, 7.5 \mathrm{mmol})$ in $35 \mathrm{~mL}$ THF/DMF 5:1 and $14 \mathrm{~mL}$ of $1 \mathrm{M}$ aqueous $\mathrm{Na}_{2} \mathrm{HPO}_{4}$ was added $\mathrm{PdCl}_{2}(1.6 \mathrm{~g}, 9 \mathrm{mmol})$ and the mixture was stirred vigorously for $24 \mathrm{~h}$. Most of the THF was removed in vacuo, brine $(25 \mathrm{~mL})$ was added and the black suspension was extracted three times with $\mathrm{Et}_{2} \mathrm{O} /$ hexane $1: 1$. The combined organic layers were dried over $\mathrm{MgSO}_{4}$ and concentrated in vacuo. The residue was purified by column chromatography (250 $\mathrm{g}$ of $\mathrm{SiO}_{2}$, hexane/EtOAc $\left.3: 1, \mathrm{R}_{\mathrm{f}}=0.20\right)$ to provide $52(2.8 \mathrm{~g}, 84 \%)$ as a colorless oil a mixture of 8 diastereomers : Anal. calcd for $\mathrm{C}_{23} \mathrm{H}_{38} \mathrm{O}_{8}$ : C 62.42; H, 8.65; found: $\mathrm{C}, 62.66 ; \mathrm{H}$, 8.49; ${ }^{1} \mathrm{H}-\mathrm{NMR}\left(250 \mathrm{MHz} ; \mathrm{CDCl}_{3}\right): \delta[\mathrm{ppm}]=4.93(\mathrm{~s}, 0.125 \mathrm{H}), 4.92(\mathrm{~s}, 0.125 \mathrm{H}), 4.91(\mathrm{~s}$, $0.125 \mathrm{H}), 4.89(\mathrm{~s}, 0.125 \mathrm{H}), 4.82(\mathrm{~s}, 0.125 \mathrm{H}), 4.80(\mathrm{~s}, 0.125 \mathrm{H}), 4.76(\mathrm{~s}, 0.125 \mathrm{H}), 4.73(\mathrm{~s}$, 0.125H), 4.69-4.78 (m, 1H), 4.58-4.66 (m, 0.5H), 4.50-4.56 (m, 0.5H), $3.78(\mathrm{~s}, 3 \mathrm{H}), 3.70-$ 3.92 (m, 3H), 3.40-3.60 (m, 2H), 2.80-3.10 (m, 2H), 2.20 (s, 1.5H), 2.16 (s, 1.5H), 1.70-1.85 $(\mathrm{m}, 4 \mathrm{H}), 1.40-1.70(\mathrm{~m}, 9 \mathrm{H}), 1.20-1.35(\mathrm{~m}, 1 \mathrm{H}), 1.15(\mathrm{~d}, J=7.1 \mathrm{~Hz}, 0.375 \mathrm{H}), 1.14(\mathrm{~d}, J=$ $6.9 \mathrm{~Hz}, 0.375 \mathrm{H}), 1.13(\mathrm{~d}, J=7.1 \mathrm{~Hz}, 0.375 \mathrm{H}), 1.12(\mathrm{~d}, J=7.1 \mathrm{~Hz}, 0.375 \mathrm{H}), 1.11(\mathrm{~d}, J=7.1$ $\mathrm{Hz}, 0.75 \mathrm{H}), 1.09$ (d, $J=7.1 \mathrm{~Hz}, 0.375 \mathrm{H}), 1.08(\mathrm{~d}, J=7.1 \mathrm{~Hz}, 0.375 \mathrm{H}), 1.04(\mathrm{~d}, J=7.1 \mathrm{~Hz}$, $0.75 \mathrm{H}), 1.03(\mathrm{~d}, J=7.1 \mathrm{~Hz}, 0.375 \mathrm{H}), 0.98(\mathrm{~d}, J=7.1 \mathrm{~Hz}, 0.75 \mathrm{H}), 0.97(\mathrm{~d}, J=7.1 \mathrm{~Hz}$, $0.75 \mathrm{H}), 0.87(\mathrm{~d}, J=7.1 \mathrm{~Hz}, 0.375 \mathrm{H}) ; \mathrm{MS}:\left(\mathrm{FI}, 7 \mathrm{kV}, 3 \mathrm{~mA}, 95^{\circ} \mathrm{C}\right) \mathrm{m} / \mathrm{z}=442[\mathrm{M}](4), 341(5)$, 269 (4), 173 (27), 101 (13), 85 (100); IR (thin film) $1 / \lambda\left[\mathrm{cm}^{-1}\right]=2942,2870,1755,1718$, 1458, 1439, 1377, 1356, 1275, 1202, 1183, 1078, 1036, 975, 906, 870, 817, 738.

Methyl $(2 R / S)-2-[(2 R, 3 R, 6 S)$-tetrahydro-2-hydroxy-3-methyl-6-\{(1S)-1-methyl-2-oxoprop-1-yl\}-2H-pyran-2-yl]-glycolate

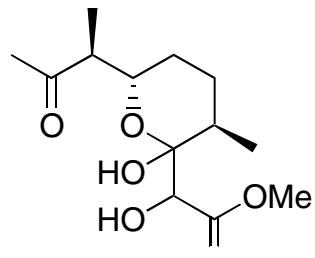


To 52 (950 mg, $2.15 \mathrm{mmol})$ in $20 \mathrm{~mL}$ of a THF/water 3:1 mixture was added PPTS (15 mg) and the mixture was warmed to $50^{\circ} \mathrm{C}$ for $12 \mathrm{~h}$. The mixture was diluted with toluene $(15 \mathrm{~mL})$ and saturated aqueous $\mathrm{NaHCO}_{3}(15 \mathrm{~mL})$. Most of the THF was removed in vacuo, the layers were separated and the aqueous layer was extracted three times with EtOAc. The combined organic layers were dried over $\mathrm{MgSO}_{4}$, filtered and concentrated in vacuo. The residue was purified by column chromatography $\left(40 \mathrm{~g}\right.$ of $\mathrm{SiO}_{2}$, hexane/EtOAc, $\left.2: 1, \mathrm{R}_{\mathrm{f}}=0.21\right)$ to provide $560 \mathrm{mg}(96 \%)$ of methyl $(2 R / S)-2-[(2 R, 3 R, 6 S)$-tetrahydro-2-hydroxy-3-methyl-6- $\{(1 S)-1$ methyl-2-oxy-propyl -2H-pyran-2-yl]-glycolate as a white amorphous solid a mixture of 2 diastereomers. Anal. calcd for $\mathrm{C}_{13} \mathrm{H}_{22} \mathrm{O}_{6}$ : C, 56.92; H, 8.08; found: C, 57.18; $\mathrm{H}, 8.35$. ${ }^{1} \mathrm{H}-$ $\operatorname{NMR}\left(250 \mathrm{MHz} ; \mathrm{CDCl}_{3}\right): \delta[\mathrm{ppm}]=4.16(\mathrm{~d}, J=9.1 \mathrm{~Hz}, 0.5 \mathrm{H}), 4.13(\mathrm{~d}, J=9.0 \mathrm{~Hz}, 0.5 \mathrm{H})$, $4.0(\mathrm{~m}, 0.5 \mathrm{H}), 3.93(\mathrm{~m}, 0.5 \mathrm{H}), 3.80(\mathrm{~s}, 1.5 \mathrm{H}), 3.78(\mathrm{~s}, 1.5 \mathrm{H}), 3.20(\mathrm{~d}, J=9.0 \mathrm{~Hz}, 0.5 \mathrm{H}), 2.98$ $(\mathrm{d}, J=9.1 \mathrm{~Hz}, 0.5 \mathrm{H}), 2.48(\mathrm{~m}, 1 \mathrm{H}), 2.03(\mathrm{~s}, 1.5 \mathrm{H}), 2.01(\mathrm{~s}, 1.5 \mathrm{H}), 1.76-1.95(\mathrm{~m}, 1 \mathrm{H}), 1.48-$ $1.75(\mathrm{~m}, 3 \mathrm{H}), 1.20-1.30(\mathrm{~m}, 1 \mathrm{H}), 0.96(\mathrm{~d}, J=6.7 \mathrm{~Hz}, 1.5 \mathrm{H}), 0.95(\mathrm{~d}, J=7.1 \mathrm{~Hz}, 3 \mathrm{H}), 0.92$ $(\mathrm{d}, J=6.6 \mathrm{~Hz}, 1.5 \mathrm{H}) ;{ }^{13} \mathrm{C}-\mathrm{NMR}\left(63 \mathrm{MHz} ; \mathrm{CDCl}_{3}\right): \delta[\mathrm{ppm}]=211.9,173.4,99.5,97.7,73.7$, $73.2,72.2,52.8,52.0,51.8,33.9,32.8,29.9,29.2,27.3,26.5,16.1,15.7,12.8,12.4 ;[\alpha]_{\mathrm{D}}^{20}=$ $+48.9^{\circ}\left(\mathrm{c}=2.0 ; \mathrm{CH}_{2} \mathrm{Cl}_{2}\right) ; \mathrm{MS}\left(\mathrm{EI}, 70 \mathrm{eV}, 25^{\circ} \mathrm{C}\right) \mathrm{m} / \mathrm{z}=255[\mathrm{M}+1](1), 206$ (3), 185 (36), 167 (36), 140 (46), 125 (100); IR (thin film): $1 / \lambda\left[\mathrm{cm}^{-1}\right]=3469,3340,2951,2937,1737,1703$, 1462, 1409, 1377, 1286, 1163, 1089, 1068, 1029, 996.

Methyl $(4 R / S, 5 S, 7 S, 10 R)-2,2,10-$ trimethyl-7-[(1S)-1-methyl-2-oxo-prop-1-yl)-1,3,6trioxaspiro[4.5]decan-4-carboxylate (44)

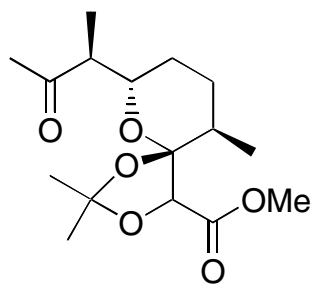

Methyl $(2 R / S)$-2-[(2R, 3R, 6S)-tetrahydro-2-hydroxy-3-methyl-6-\{(1S)-1-methyl-2-oxo-prop1-yl -2H-pyran-2-yl]-glycolate $(0.77 \mathrm{~g}, 2.8 \mathrm{mmol})$ was dissolved in acetone $(30 \mathrm{~mL})$ (previously dried over potassium carbonate and distilled) and a small amount of phosphorus 
pentoxide was added. The reaction mixture was stirred at room temperature for $12 \mathrm{~h}$. A second portion of phosphorus pentoxide was added and stirring was continued for an additional $10 \mathrm{~h}$. The mixture was diluted with toluene $(15 \mathrm{~mL})$ and saturated aqueous $\mathrm{NaHCO}_{3}(15 \mathrm{~mL})$. Most of the acetone was removed in vacuo, the layers were separated and the aqueous layer was extracted three times with $\mathrm{Et}_{2} \mathrm{O}$. The combined organic layers were washed with brine, dried over $\mathrm{MgSO}_{4}$, filtered and concentrated in vacuo. The residue was purified by column chromatography $\left(60 \mathrm{~g}\right.$ of $\mathrm{SiO}_{2}$, hexane/EtOAc, $\left.2: 1, \mathrm{R}_{\mathrm{f}}=0.41\right)$ to provide $44(0.61 \mathrm{~g}, 69 \%)$ as a colorless oil a mixture of 2 diastereomers: Anal. calcd for $\mathrm{C}_{16} \mathrm{H}_{26} \mathrm{O}_{6}: \mathrm{C}$ 61.13; H, 8.34; found: C, 61.32; H, 8.35; ${ }^{1} \mathrm{H}-\mathrm{NMR}\left(400 \mathrm{MHz} ; \mathrm{CDCl}_{3}\right): \delta[\mathrm{ppm}]=4.59(\mathrm{~s}$, $0.5 \mathrm{H}), 4.53(\mathrm{~s}, 0.5 \mathrm{H}), 3.98(\mathrm{ddd}, J=11.2,8.7,2.3 \mathrm{~Hz}, 0.5 \mathrm{H}), 3.91$ (ddd, $J=10.3,8.0,2.1$ $\mathrm{Hz}, 0.5 \mathrm{H}), 3.83(\mathrm{~s}, 1.5 \mathrm{H}), 3.80(\mathrm{~s}, 1.5 \mathrm{H}), 2.69(\mathrm{dq}, J=8.4,7.0 \mathrm{~Hz}, 0.5 \mathrm{H}), 2.58(\mathrm{dq}, J=7.9$, $7.0 \mathrm{~Hz}, 0.5 \mathrm{H}), 2.21(\mathrm{~s}, 1.5 \mathrm{H}), 2.16(\mathrm{~s}, 1.5 \mathrm{H}), 2.03(\mathrm{ddq}, J=10.9,6.7,4.3 \mathrm{~Hz}, 0.5 \mathrm{H}), 1.91$ (ddq, $J=10.7,6.6,4.2 \mathrm{~Hz}, 0.5 \mathrm{H}), 1.75-1.50$ (m, 3H), 1.53 (s, 3H), 1.44 (s, 1.5H), 1.41 (s, $1.5 \mathrm{H}), 1.35-1.25(\mathrm{~m}, 1 \mathrm{H}), 1.03(\mathrm{~d}, J=7.0 \mathrm{~Hz}, 1.5 \mathrm{H}), 0.99(\mathrm{~d}, J=7.0 \mathrm{~Hz}, 1.5 \mathrm{H}), 0.98(\mathrm{~d}, J=$ $6.7 \mathrm{~Hz}, 1.5 \mathrm{H}), 0.75(\mathrm{~d}, J=6.6 \mathrm{~Hz}, 1.5 \mathrm{H}) ;{ }^{13} \mathrm{C}-\mathrm{NMR}\left(63 \mathrm{MHz} ; \mathrm{CDCl}_{3}\right): \delta[\mathrm{ppm}]=212.0$, $211.8,169.3,168.5,112.5,111.1,107.7,107.1,84.1,80.1,74.7,73.7,52.2,51.9,51.6,51.3$, $31.1,30.7,28.7,28.5,28.2,28.1,27.8,26.9,26.7,25.5,16.1,15.8,12.6,12.4$; MS: (FI, $\left.7 \mathrm{kV}, 3 \mathrm{~mA}, 40^{\circ} \mathrm{C}\right) \mathrm{m} / \mathrm{z}=314[\mathrm{M}](10), 299[\mathrm{M}-15](100) ;[\alpha]_{\mathrm{D}}^{20}=+75.0^{\circ}\left(\mathrm{c}=2 ; \mathrm{CH}_{2} \mathrm{Cl}_{2}\right) ; \mathrm{IR}$ (thin film) $1 / \lambda\left[\mathrm{cm}^{-1}\right]=2944,2882,1765,1738,1715,1456,1440,1381,1287,1207,1189$, $1118,1072,1033,991,963$.

Methyl $(4 R / S, 5 S, 7 S, 10 R)$-2,2,10-trimethyl-7-[(1R, 4S, 7E, 9Z, 13S)-4-hydroxy-1methyl-2-oxo-13-tert-butyldimethylsilyloxy-tetradeca-7,9-dien-1-yl]-1,3,6-

trioxaspiro[4.5]decan-4-carboxylate (53)

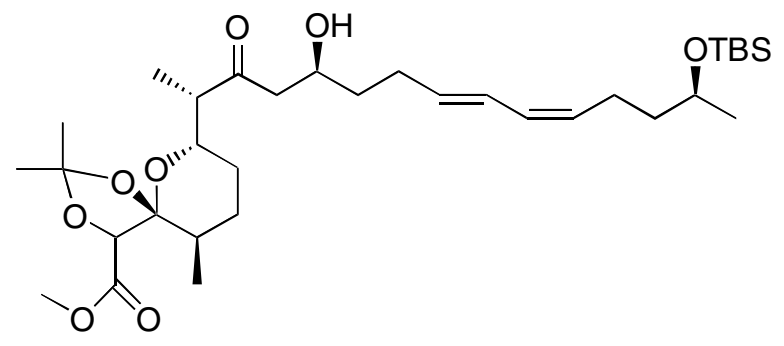


(-)-Chlorodiisopinocampheylborane $(560 \mathrm{mg}, 1.74 \mathrm{mmol})$ was dissolved in $5 \mathrm{~mL}$ of THF. $\mathrm{Et}_{3} \mathrm{~N}(0.26 \mathrm{~mL}, 1.83 \mathrm{mmol})$ was added and the mixture was cooled to $-78^{\circ} \mathrm{C}$. Over a period of $15 \mathrm{~min}, 44$ (520 mg, $1.66 \mathrm{mmol})$ in $5 \mathrm{~mL}$ THF was added and the mixture was stirred for further $4 \mathrm{~h}$ at $-40^{\circ} \mathrm{C}$. After this time, a white precipitate had formed and the mixture was cooled to $-78^{\circ} \mathrm{C}$. Then aldehyde $4 \mathbf{a}(540 \mathrm{mg}, 1.83 \mathrm{mmol})$ in $5 \mathrm{~mL}$ of THF was added and the mixture was stored for $60 \mathrm{~h}$ at $-78^{\circ} \mathrm{C}$ in a freezer. Saturated aqueous $\mathrm{NH}_{4} \mathrm{Cl}(15 \mathrm{~mL})$ was added and the mixture was warmed to room temperature. The layers were separated and aqueous layer extracted three times with ether. The combined organic layers were concentrated in vacuo, the residue was dissolved in $30 \mathrm{~mL}$ of ice cold pentane and shaken for 10 min with $\mathrm{H}_{2} \mathrm{O}_{2}(0.8 \mathrm{~mL}, 30 \%, 7 \mathrm{mmol})$ in $10 \mathrm{~mL}$ of $0.5 \mathrm{M}$ aqu. $\mathrm{NaOH}$. The layers were separated and aqueous layer extracted three times with ether. The combined organic layers were washed with saturated aqueous $\mathrm{NH}_{4} \mathrm{Cl}$, brine, dried over $\mathrm{MgSO}_{4}$, filtered and concentrated in vacuo. The residue was purified by column chromatography (40 $\mathrm{g}$ of $\mathrm{SiO}_{2}$, hexane/EtOAc $\left.3: 1, \mathrm{R}_{\mathrm{f}}=0.39\right)$ to provide $730 \mathrm{mg}(72 \%)$ of $\mathbf{5 3}$ as 4 diastereomers in a $4: 1$ ratio with respect to the $\mathrm{C}-11$ center, which could not be separated. Data given for the two main diastereomers: Anal. calcd for $\mathrm{C}_{33} \mathrm{H}_{58} \mathrm{O}_{8} \mathrm{Si}$ : C 64.88; H, 9.57. Found: C, 65.17; H, 9.39. ${ }^{1} \mathrm{H}-\mathrm{NMR}\left(400 \mathrm{MHz} ; \mathrm{CDCl}_{3}\right): \delta[\mathrm{ppm}]=6.33(\mathrm{dd}, J=15.2,10.9 \mathrm{~Hz}, 0.5 \mathrm{H}), 6.32(\mathrm{dd}, J=$ $15.2,10.9 \mathrm{~Hz}, 0.5 \mathrm{H}), 5.93(\mathrm{dd}, J=10.9,10.7 \mathrm{~Hz}, 0.5 \mathrm{H}), 5.91(\mathrm{dd}, J=10.9,10.7 \mathrm{~Hz}, 0.5 \mathrm{H})$, $5.68(\mathrm{dt}, J=15.2,6.9 \mathrm{~Hz}, 0.5 \mathrm{H}), 5.63(\mathrm{dt}, J=15.2,6.9 \mathrm{~Hz}, 0.5 \mathrm{H}), 5.30(\mathrm{dt}, J=10.7,7.4 \mathrm{~Hz})$, $5.28(\mathrm{dt}, J=10.7,7.4 \mathrm{~Hz}), 4.53(\mathrm{~s}, 0.5 \mathrm{H}), 4.42(\mathrm{~s}, 0.5 \mathrm{H}), 3.97-4.07(\mathrm{~m}, 1 \mathrm{H}), 3.82-3.95(\mathrm{~m}$, 2H), 3.79 (s, $1.5 \mathrm{H}), 3.75$ (s, $1.5 \mathrm{H}), 2.43-2.64$ (m, $3 \mathrm{H}), 2.08-2.35$ (m, $4 \mathrm{H}), 1.97$ (dqd, $J=$ $10.5,7.0,5.7 \mathrm{~Hz}, 0.5 \mathrm{H}), 1.86(\mathrm{dqd}, J=11.0,6.5,3.7 \mathrm{~Hz}, 0.5 \mathrm{H}), 1.60-1.72(\mathrm{~m}, 3 \mathrm{H}), 1.50(\mathrm{~s}$, $1.5 \mathrm{H}), 1.45(\mathrm{~s}, 1.5 \mathrm{H}), 1.38(\mathrm{~s}, 1.5 \mathrm{H}), 1.36(\mathrm{~s}, 1.5 \mathrm{H}), 1.35-1.58(\mathrm{~m}, 4 \mathrm{H}), 1.20-1.33(\mathrm{~m}, 1 \mathrm{H})$, $1.13(\mathrm{~d}, J=7.0 \mathrm{~Hz}, 3 \mathrm{H}), 0.99$ (d, $J=7.0 \mathrm{~Hz}, 1.5 \mathrm{H}), 0.95$ (d, $J=7.0 \mathrm{~Hz}, 1.5 \mathrm{H}), 0.92$ (d, $J=$ $7.0 \mathrm{~Hz}, 1.5 \mathrm{H}), 0.71(\mathrm{~d}, J=6.5 \mathrm{~Hz}, 1.5 \mathrm{H}), 0.04(\mathrm{~s}, 6 \mathrm{H}) ;{ }^{13} \mathrm{C}-\mathrm{NMR}\left(63 \mathrm{MHz} ; \mathrm{CDCl}_{3}\right): \delta[\mathrm{ppm}]$ = 215.2, 215.1, 169.2, 168.2, 112.5 134.1, 133.5, 130.1, 129.8, 128.6, 128.5, 126.1, 125.9, 112.5, 111.0, 107.7, 106.9, 84.0, 79.8, 74.9, 73.8, 68.2, 66.9, 66.8, 52.2, 52.0, 51.9, 51.3, 51.0, 50.6, 31.1, 30.7, 28.7, 28.5, 28.3, 28.1, 27.8, 26.9, 26.7, 25.5, 16.1, 15.7, 12.6, 12.4, 4.4, -4.7; MS: (FI, $7 \mathrm{kV}, 3 \mathrm{~mA}, 170^{\circ} \mathrm{C}$ ) m/z =610 (100), 611 (28), 374 (45); IR (thin film): $1 / \lambda\left[\mathrm{cm}^{-1}\right]=3544,2931,2857,1768,1739,1710,1459,1375,1255,1217,1120$, 1074, 1008, 836, 774 . 
Methyl $(4 R / S, 5 S, 7 S, 10 R)-2,2,10$-trimethyl-7-[(1R, 4S, 7E, 9Z, 13S) -13-tertbutyldimethylsilyloxy-4-methoxymethyloxy-1-methyl-2-oxo-tetradeca-7,9-dien-1-yl]1,3,6-trioxaspiro[4.5]decan-4-carboxylate (54)

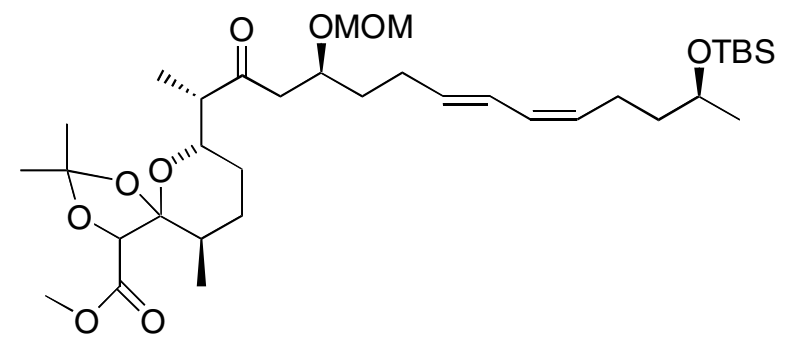

To a stirred solution of $\mathbf{5 3}(100 \mathrm{mg}, 0.16 \mathrm{mmol})$ in $6 \mathrm{~mL}$ of $\mathrm{CH}_{2} \mathrm{Cl}_{2}$ and Hünig's base (130 $\mathrm{mg}, 1 \mathrm{mmol})$ was added $\mathrm{MOMCl}(54 \mathrm{mg}, 0.65 \mathrm{mmol})$. The reaction mixture was stirred for $30 \mathrm{~h}$ and satd. aqu. $\mathrm{NH}_{4} \mathrm{Cl}(8 \mathrm{~mL})$ was added. The layers were separated and the aqueous layer extracted three times with ether. The combined organic layers were washed with brine, dried over $\mathrm{MgSO}_{4}$, filtered, and concentrated in vacuo. The crude product was purified by column chromatography (40 g of $\mathrm{SiO}_{2}$, hexanes/EtOAc $\left.5: 1 \mathrm{R}_{\mathrm{f}}=0.30\right)$ to provide $95 \mathrm{mg}(90$ $\%$ ) of 54 as a mixture of 2 main diastereomers : Anal. calcd for $\mathrm{C}_{35} \mathrm{H}_{62} \mathrm{O}_{9} \mathrm{Si}: \mathrm{C} 64.18 ; \mathrm{H}$, 9.54; found: C, 63.98; H, 9.40. ${ }^{1} \mathrm{H}-\mathrm{NMR}\left(400 \mathrm{MHz} ; \mathrm{CDCl}_{3}\right): \delta[\mathrm{ppm}]=6.31(\mathrm{dd}, J=15.0$, $11.1 \mathrm{~Hz}, 0.5 \mathrm{H}), 6.30(\mathrm{dd}, J=15.0,11.1 \mathrm{~Hz}, 0.5 \mathrm{H}), 5.91(\mathrm{dd}, J=11.1,11.0 \mathrm{~Hz}, 0.5 \mathrm{H}), 5.89$ $(\mathrm{dd}, J=11.1,11.0 \mathrm{~Hz}, 0.5 \mathrm{H}), 5.65(\mathrm{dt}, J=15.0,6.9 \mathrm{~Hz}, 0.5 \mathrm{H}), 5.60(\mathrm{dt}, J=15.0,6.9 \mathrm{~Hz}$, $0.5 \mathrm{H}), 5.29(\mathrm{dt}, J=11.0,7.5 \mathrm{~Hz}, 0.5 \mathrm{H}), 5.25(\mathrm{dt}, J=11.0,7.5 \mathrm{~Hz}, 0.5 \mathrm{H}), 4.67(\mathrm{~d}, J=2.0 \mathrm{~Hz}$, $0.5 \mathrm{H}), 4.65(\mathrm{~d}, J=2.0 \mathrm{~Hz}, 0.5 \mathrm{H}), 4.59(\mathrm{~d}, J=4.5 \mathrm{~Hz}, 0.5 \mathrm{H}), 4.57(\mathrm{~d}, J=4.5 \mathrm{~Hz}, 0.5 \mathrm{H}), 4.52$ (s, 0.5H), $4.43(\mathrm{~s}, 0.5 \mathrm{H}), 3.97-4.07(\mathrm{~m}, 1 \mathrm{H}), 3.95$ (ddd, $J=11.5,8.7,2.0 \mathrm{~Hz}, 0.5 \mathrm{H}), 3.86$ (ddd, $J=11.5,8.7,2.0 \mathrm{~Hz}, 0.5 \mathrm{H}), 3.76-3.84$ (m, 1H), 3.79 (s, 1.5H), 3.75 (s, 1.5H), 3.33 (s, $1.5 \mathrm{H}), 3.32(\mathrm{~s}, 1.5 \mathrm{H}), 2.82(\mathrm{dd}, J=17.0,7.0 \mathrm{~Hz}, 0.5 \mathrm{H}), 2.75(\mathrm{dd}, J=17.0,7.5 \mathrm{~Hz}, 0.5 \mathrm{H})$, $2.65(\mathrm{dq}, J=8.5,7.0 \mathrm{~Hz}, 0.5 \mathrm{H}), 2.48-2.60(\mathrm{~m}, 1.5 \mathrm{H}), 2.08-2.28(\mathrm{~m}, 4 \mathrm{H}), 1.98(\mathrm{dqd}, J=$ 10.4, 6.5, $5.8 \mathrm{~Hz}, 0.5 \mathrm{H}), 1.85$ (dqd, $J=11.1,6.5,3.6 \mathrm{~Hz}, 0.5 \mathrm{H}), 1.60-1.72$ (m, $3 \mathrm{H}), 1.49$ (s, 1.5H), 1.44 (s, 1.5H), $1.38(\mathrm{~s}, 1.5 \mathrm{H}), 1.35(\mathrm{~s}, 1.5 \mathrm{H}), 1.35-1.58(\mathrm{~m}, 4 \mathrm{H}), 1.20-1.33(\mathrm{~m}, 1 \mathrm{H})$, $1.12(\mathrm{~d}, J=6.0 \mathrm{~Hz}, 3 \mathrm{H}), 0.96(\mathrm{~d}, J=7.0 \mathrm{~Hz}, 1.5 \mathrm{H}), 0.94(\mathrm{~d}, J=6.5 \mathrm{~Hz}, 1.5 \mathrm{H}), 0.91$ (d, $J=$ $6.5 \mathrm{~Hz}, 1.5 \mathrm{H}), 0.88(\mathrm{~s}, 9 \mathrm{H}), 0.70(\mathrm{~d}, J=6.5 \mathrm{~Hz}, 1.5 \mathrm{H}), 0.04(\mathrm{~s}, 6 \mathrm{H}) ;{ }^{13} \mathrm{C}-\mathrm{NMR}(63 \mathrm{MHz}$; $\left.\mathrm{CDCl}_{3}\right): \delta[\mathrm{ppm}]=212.3,211.8,169.6,168.6,134.2,133.8,130.4,130.1,128.9,128.8$, $126.3,126.2,112.7,111.4,108.1,107.3,96.6,84.4,80.2,74.9,74.0,73.8,68.5,55.9,52.5$, 
52.1, 51.8, 51.7, 50.5, 51.0, 40.0, 35.1, 35.0, 34.1, 34.0, 29.4, 29.0, 28.7, 28.1, 28.0, 27.9, 27.1, 27.0, 26.2, 25.8, 24.1, 24.0, 16.4, 16.0, 12.7, 12.2, -3.9, -4.3; MS: (FI, 7kV, 3mA, $\left.170^{\circ} \mathrm{C}\right) \mathrm{m} / \mathrm{z}=654[\mathrm{M}](100), 597(18), 391$ (13); IR (thin film): $1 / \lambda\left[\mathrm{cm}^{-1}\right]=2932,2857$, 1768, 1739, 1717, 1460, 1438, 1376, 1255, 1216, 1150, 1120, 1093, 1043, 1008, 919, 885, 836,775 .

Methyl (4R/S, 5S, 7S, 10R)-2,2,10-trimethyl-7-[(1R, 2R/S, 4S, 7E, 9Z, 13S) -13-tertbutyldimethylsilyloxy-2-hydroxy-4-methoxymethyloxy-1-methyl-tetradeca-7,9-dien-1yl]-1,3,6-trioxaspiro[4.5]decan-4-carboxylate (55)

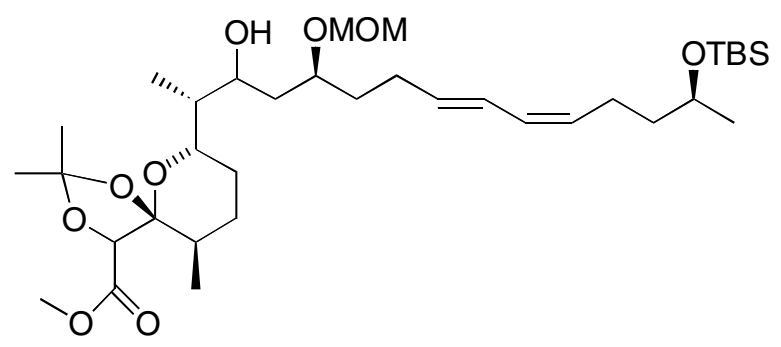

To a stirred solution of 54 (405 mg $0.62 \mathrm{mmol})$ in $\mathrm{MeOH}(10 \mathrm{~mL})$ and THF $(2 \mathrm{~mL})$ at $-20^{\circ} \mathrm{C}$ was added sodium borohydride $(36 \mathrm{mg}, 0.93 \mathrm{mmol}$ ). The reaction mixture was stirred for $2 \mathrm{~h}$ allowing the temperature to rise to $0^{\circ} \mathrm{C}$ and then for $2 \mathrm{~h}$ at $0^{\circ} \mathrm{C}$. Upon quenching with acetone $(1 \mathrm{~mL})$ the reaction mixture was diluted with brine $(10 \mathrm{~mL})$ and ether $(15 \mathrm{~mL})$. The layers were separated and the aqueous layer extracted three times with ether. The combined organic extracts were dried over $\mathrm{MgSO}_{4}$, filtered, and concentrated in vacuo. The crude product was purified by column chromatography $\left(60 \mathrm{~g}\right.$ of $\mathrm{SiO}_{2}$, hexane/EtOAc, $\left.3: 1\right)$ to provide $102 \mathrm{mg}$ $\left(0.16 \mathrm{mmol}\right.$ ) of a minor product (two diastereomers $\mathrm{R}_{\mathrm{f}}=0.35$ ) and $263 \mathrm{mg}$ of a main product (two diastereomers $\mathrm{R}_{\mathrm{f}}=0.31$ ) (89\% combined yield). HPLC separation delivered all 4 main diastereomers in the analytically pure forms. HPLC data for Merck Supersphere ${ }^{\circledR}$ Si $604 \mu \mathrm{m}$, $244 \mathrm{~mm} \times 4 \mathrm{~mm}$ column, $2 \% \mathrm{i}-\mathrm{PrOH}$ in hexane, flow: $2 \mathrm{~mL} / \mathrm{min}$. Compound 1 55a, $\mathrm{t}_{\mathrm{R}}=4.4$ min; compound $2 \mathbf{5 5 b}, t_{R}=5.4 \mathrm{~min}$; compound $3 \mathbf{5 5 c}, t_{R}=6.5 \mathrm{~min}$; compound $4 \mathbf{5 5 d}, \mathrm{t}_{\mathrm{R}}=$ 7.5 min. Analytical data given for compound 1 55a. Anal. calcd for $\mathrm{C}_{35} \mathrm{H}_{64} \mathrm{O}_{9} \mathrm{Si}$ : C 63.99; $\mathrm{H}$, 9.82; found: C, 63.93; $\mathrm{H}, 9.58 .{ }^{1} \mathrm{H}-\mathrm{NMR}\left(400 \mathrm{MHz} ; \mathrm{CDCl}_{3}\right): \delta[\mathrm{ppm}]=6.31(\mathrm{dd}, 15.1,11.0$ $\mathrm{Hz}, 1 \mathrm{H}), 5.92(\mathrm{dd}, J=11.0,10.5 \mathrm{~Hz}, 1 \mathrm{H}), 5.65$ (dt, $J=15.1,6.8 \mathrm{~Hz}, 1 \mathrm{H}), 5.30$ (dt, $J=10.5$, $7.5 \mathrm{~Hz}, 1 \mathrm{H}), 4.65(\mathrm{~s}, 2 \mathrm{H}), 4.59(\mathrm{~s}, 1 \mathrm{H}), 4.00(\mathrm{~m}, 1 \mathrm{H}), 3.72-3.86(\mathrm{~m}, 2 \mathrm{H}), 3.79(\mathrm{~s}, 3 \mathrm{H}), 3.38$ (s, 3H), 2.08-2.30 (m, 4H), $1.98(\mathrm{dqd}, J=11.0,6.5,4.5 \mathrm{~Hz}, 1 \mathrm{H}), 1.79(\mathrm{ddd}, J=14.1,9.0,7.0$ 
Hz, 1H), $1.35-1.74(\mathrm{~m}, 11 \mathrm{H}), 1.68(\mathrm{~s}, 3 \mathrm{H}), 1.40(\mathrm{~s}, 3 \mathrm{H}), 1.13(\mathrm{~d}, J=6.5 \mathrm{~Hz}, 3 \mathrm{H}), 0.97$ (d, $J$ $=6.5 \mathrm{~Hz}, 3 \mathrm{H}), 0.89(\mathrm{~s}, 9 \mathrm{H}), 0.89(\mathrm{~d}, J=6.0 \mathrm{~Hz}, 3 \mathrm{H}), 0.04(\mathrm{~s}, 6 \mathrm{H}) ;{ }^{13} \mathrm{C}-\mathrm{NMR}(63 \mathrm{MHz}$; $\left.\mathrm{CDCl}_{3}\right): \delta[\mathrm{ppm}]=168.9,133.9,130.0,128.5,125.9,112.5,107.5,95.4,80.4,76.1,74.5$, $68.2,68.1,55.7,52.2,42.7,39.9,39.2,34.2,34.0,29.0,28.4,28.3,27.9,26.9,25.9,23.8$, $23.7,18.1,16.2,10.5,-4.4,-4.7 ;[\alpha]_{\mathrm{D}}^{20}=31.0^{\circ}\left(\mathrm{c}=2.0 ; \mathrm{CHCl}_{3}\right)$; MS: (FI, 7kV, $\left.3 \mathrm{~mA}, 150^{\circ} \mathrm{C}\right)$ $\mathrm{m} / \mathrm{z}=656[\mathrm{M}]$ (73), 530 (100), 115 (48); IR (thin film): $1 / \lambda\left[\mathrm{cm}^{-1}\right]=3524,2931,2858$, 1767, 1740, 1460, 1381, 1255, 1217, 1124, 1094, 1035, 919, 886, 837, 775.

Methyl $(5 S, 7 S, 10 R)-2,2,10$-trimethyl-7-[(1R, 4S, 7E, 9Z, 13S)-2,13-dihydroxy-4methoxymethyloxy-1-methyl-tetradeca-7,9-dien-1-yl]-1,3,6-trioxaspiro[4.5]decan-4carboxylate (56)

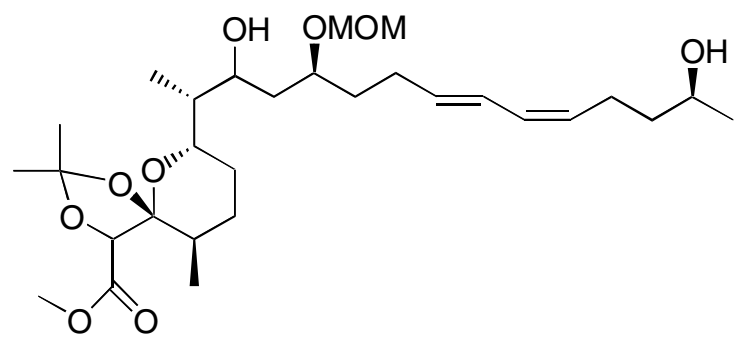

A stock solution of $5 \mathrm{~mL} \mathrm{HF} /$ pyridine complex, $10 \mathrm{~mL}$ pyridine and $15 \mathrm{~mL}$ THF was prepared. In a Teflon vessel, 55a $(130 \mathrm{mg}, 0.20 \mathrm{mmol})$ was dissolved in THF (3 mL) and 1.3 $\mathrm{mL}$ of the stock solution was added slowly at ambient temperature. The mixture was stirred for $24 \mathrm{~h}$ and then diluted with $\mathrm{Et}_{2} \mathrm{O}(15 \mathrm{~mL})$ and saturated aqueous $\mathrm{CuSO}_{4}(10 \mathrm{~mL})$. The phases were separated and the aqueous layer was extracted three times with EtOAc ( 3 x 20 $\mathrm{mL}$ ). The combined organic layers were washed with saturated aqueous $\mathrm{NaHCO}_{3}$, dried over $\mathrm{MgSO}_{4}$ and concentrated in vacuo. The residue was purified by column chromatography (25 $\mathrm{g} \mathrm{SiO}_{2}$, hexane/EtOAc, $\left.1: 1, \mathrm{R}_{\mathrm{f}}=0.19\right)$ to provide $56(101 \mathrm{mg}, 94 \%)$ as a clear colorless oil. Anal. calcd for $\mathrm{C}_{29} \mathrm{H}_{50} \mathrm{O}_{9} \mathrm{Si}$ : C 64.18; H, 9.29; found: C, 63.93; H, 9.05. ${ }^{1} \mathrm{H}-\mathrm{NMR}(400 \mathrm{MHz}$; $\left.\mathrm{CDCl}_{3}\right): \delta[\mathrm{ppm}]=6.32(\mathrm{dd}, J=14.8,11.0 \mathrm{~Hz}, 1 \mathrm{H}), 5.92(\mathrm{dd}, J=11.0,11.0 \mathrm{~Hz}, 1 \mathrm{H}), 5.64$ $(\mathrm{dt}, J=14.8,7.0 \mathrm{~Hz}, 1 \mathrm{H}), 5.31(\mathrm{dt}, J=11.0,7.5 \mathrm{~Hz}, 1 \mathrm{H}), 4.66(\mathrm{~s}, 2 \mathrm{H}), 3.99(\mathrm{~m}, 1 \mathrm{H}), 3.72-$ $3.86(\mathrm{~m}, 3 \mathrm{H}), 3.75(\mathrm{~s}, 3 \mathrm{H}), 3.37(\mathrm{~s}, 3 \mathrm{H}), 2.59(\mathrm{~m}, 1 \mathrm{H}), 2.12-2.30(\mathrm{~m}, 4 \mathrm{H}), 1.96(\mathrm{~m}, 1 \mathrm{H}), 1.79$ (ddd, $J=14.5,9.1,6.6 \mathrm{~Hz}, 1 \mathrm{H}), 1.35-1.72(\mathrm{~m}, 10 \mathrm{H}), 1.62$ (s, 3H), 1.40 (s, 3H), 1.18 (d, $J=$ $6.2 \mathrm{~Hz}, 3 \mathrm{H}), 0.97(\mathrm{~d}, J=6.6 \mathrm{~Hz}, 3 \mathrm{H}), 0.87(\mathrm{~d}, J=7.0 \mathrm{~Hz}, 3 \mathrm{H}) ;{ }^{13} \mathrm{C}-\mathrm{NMR}\left(63 \mathrm{MHz} ; \mathrm{CDCl}_{3}\right)$ : $\delta[\mathrm{ppm}]=168.9,134.3,129.4,129.1,125.8,112.5,107.6,95.5,80.4,76.0,74.5,68.2,67.5$, 55.7, 52.2, 42.8, 39.3, 39.0, 34.2, 33.8, 29.0, 28.4, 28.3, 27.9, 26.9, 25.9, 24.1, 23.5, 16.2, 
10.5; MS: (FI, $\left.7 \mathrm{kV}, 3 \mathrm{~mA}, 100^{\circ} \mathrm{C}\right) \mathrm{m} / \mathrm{z}=542[\mathrm{M}](100) ;[\alpha]_{\mathrm{D}}^{20}=+31.0^{\circ}\left(\mathrm{c}=2.0, \mathrm{CHCl}_{3}\right)$; IR (thin film): $1 / \lambda[\mathrm{cm}-1]=3469,2936,2858,1765,1739,1439,1382,1287,1217,1149$, $1121,1033,989,919$.

\section{Monolactone (58)}

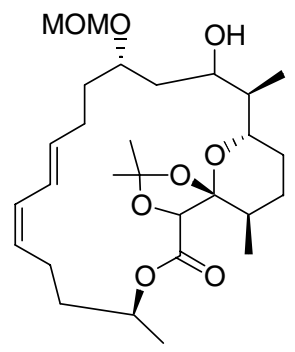

$56(12 \mathrm{mg}, 22 \mu \mathrm{mol})$ was dissolved in $\mathrm{MeOH}(1 \mathrm{~mL})$ and powdered $\mathrm{Ba}(\mathrm{OH})_{2} \cdot 8 \mathrm{H}_{2} \mathrm{O}(200 \mathrm{mg}$, $0.63 \mathrm{mmol}$ ) was added. The white suspension was vigorously stirred for $1 \mathrm{~h}$, then diluted with $5 \mathrm{~mL} \mathrm{Et}_{2} \mathrm{O}$ and $5 \mathrm{~mL}$ brine and acidified to $\mathrm{pH} 3$ with $1 \mathrm{M} \mathrm{HCl}(1.4 \mathrm{ml})$. The two clear phases were separated and aqueous layer was extracted four times with EtOAc. The combined organic extracts were washed with brine, dried over $\mathrm{MgSO}_{4}$ and concentrated in vacuo. The crude seco acid $\mathbf{5 7}$ was used for macrolactonization without any further purification. A stock solution was prepared by dissolving 2,4,6-trichlorobenzoyl chloride (96 mg, $400 \mu \mathrm{mol}$ ) and triethylamine ( $44 \mathrm{mg}, 440 \mu \mathrm{mol})$ in $2 \mathrm{~mL}$ toluene. A portion of the stock solution $(0.1 \mathrm{~mL})$ was added to the seco acid in $0.5 \mathrm{~mL}$ toluene. After stirring for $2 \mathrm{~h}$ the mixture was diluted with $0.4 \mathrm{ml}$ toluene and added over $1 \mathrm{~h}$ via syringe pump to a solution of DMAP (24 mg, 200 $\mu \mathrm{mol})$ in $2 \mathrm{~mL}$ toluene at room temperature. The suspension was stirred for an additional 0.5 h, diluted with EtOAc $(10 \mathrm{~mL})$ and saturated aqueous $\mathrm{NaHCO}_{3}(5 \mathrm{~mL})$. The phases were separated and aqueous layer was extracted three times with EtOAc. Combined organic extracts were washed with brine, dried over $\mathrm{MgSO}_{4}$ and concentrated in vacuo. The residue was purified by column chromatography ( $8 \mathrm{~g}$ of $\mathrm{SiO}_{2}$, hexane/EtOAc, $3: 1$ to $1: 1$ ) to provide $9 \mathrm{mg}\left(80 \%, \mathrm{R}_{\mathrm{f}}=0.25\right.$ hexane/EtOAc $\left.3: 1\right)$ of the monolactone 58 and $1 \mathrm{mg}\left(9 \%, \mathrm{R}_{\mathrm{f}}\right.$ $=0.25$ hexane/EtOAc $3: 1)$ of diolide 62 as clear colorless oils. Analytical data given for the monolide 58: Anal. calcd. for $\mathrm{C}_{28} \mathrm{H}_{46} \mathrm{O}_{8}$ : C, 65.86; H, 9.08; found: C, 70.19; $\mathrm{H}, 9.30 .{ }^{1} \mathrm{H}-$ $\operatorname{NMR}\left(400 \mathrm{MHz} ; \mathrm{CDCl}_{3}\right): \delta[\mathrm{ppm}]=6.31(\mathrm{dd}, J=15.4,11.1 \mathrm{~Hz}, 1 \mathrm{H}), 6.06(\mathrm{dd}, J=11.1$, $10.2 \mathrm{~Hz}, 1 \mathrm{H}), 5.67$ (ddd, $J=15.4,8.2,5.7 \mathrm{~Hz}, 1 \mathrm{H}), 5.40(\mathrm{dt}, J=10.2,7.8 \mathrm{~Hz}, 1 \mathrm{H}), 4.86$ (dqd, $J=9.6,6.4,3.8 \mathrm{~Hz}, 1 \mathrm{H}), 4.67(\mathrm{~d}, J=6.6 \mathrm{~Hz}, 1 \mathrm{H}), 4.64(\mathrm{~d}, J=6.6 \mathrm{~Hz}, 1 \mathrm{H}), 4.52(\mathrm{~s}$, 1H) $4.07(\mathrm{dq}, J=10.1,1.8 \mathrm{~Hz}, 1 \mathrm{H}), 3.76-3.88(\mathrm{~m}, 2 \mathrm{H}), 3.38(\mathrm{~s}, 3 \mathrm{H}), 2.92$ (d, $J=2.5 \mathrm{~Hz}$, 
1H), 2.27-2.38 (m, 1H), 1.90-2.25 (m, 5H), 1.54-1.80 (m, H), $1.61(\mathrm{~s}, 3 \mathrm{H}), 1.40(\mathrm{~s}, 3 \mathrm{H})$, $1.25-1.58(\mathrm{~m}, 4 \mathrm{H}), 1.13(\mathrm{~d}, J=7.0 \mathrm{~Hz}, 3 \mathrm{H}), 0.97$ (d, $J=6.5 \mathrm{~Hz}, 1.5 \mathrm{H}), 0.84$ (d, $J=7.0 \mathrm{~Hz}$, $1.5 \mathrm{H}), 0.76(\mathrm{~d}, J=7.0 \mathrm{~Hz}, 1.5 \mathrm{H}), 0.74(\mathrm{~d}, J=6.5 \mathrm{~Hz}, 1.5 \mathrm{H}) ;{ }^{13} \mathrm{C}-\mathrm{NMR}\left(63 \mathrm{MHz} ; \mathrm{CDCl}_{3}\right): \delta$ $[\mathrm{ppm}]=168.1,133.3,130.0,128.5,126.7,112.2,107.4,95.2,80.4,76.6,72.7,71.8,68.5$, $55.8,44.3,39.6,36.2,34.6,33.1,29.6,28.5,28.4,27.8,27.0,23.7,19.8,16.2,9.5$; MS: (FAB, NBA, CsI) m/z = 643 (100); HRMS (FAB) calcd for $\mathrm{C}_{28} \mathrm{H}_{46} \mathrm{O}_{8} \mathrm{Cs}$, [M+Cs] :643.2247 found: 643.2244 ; IR (thin film): $1 / \lambda\left[\mathrm{cm}^{-1}\right]=3545,2937,1761,1729,1459,1380,1280$, 1219, 1153, 1119, 1092, 1036, 1003, 954, 923, 737.

\section{Protected dimer (59)}

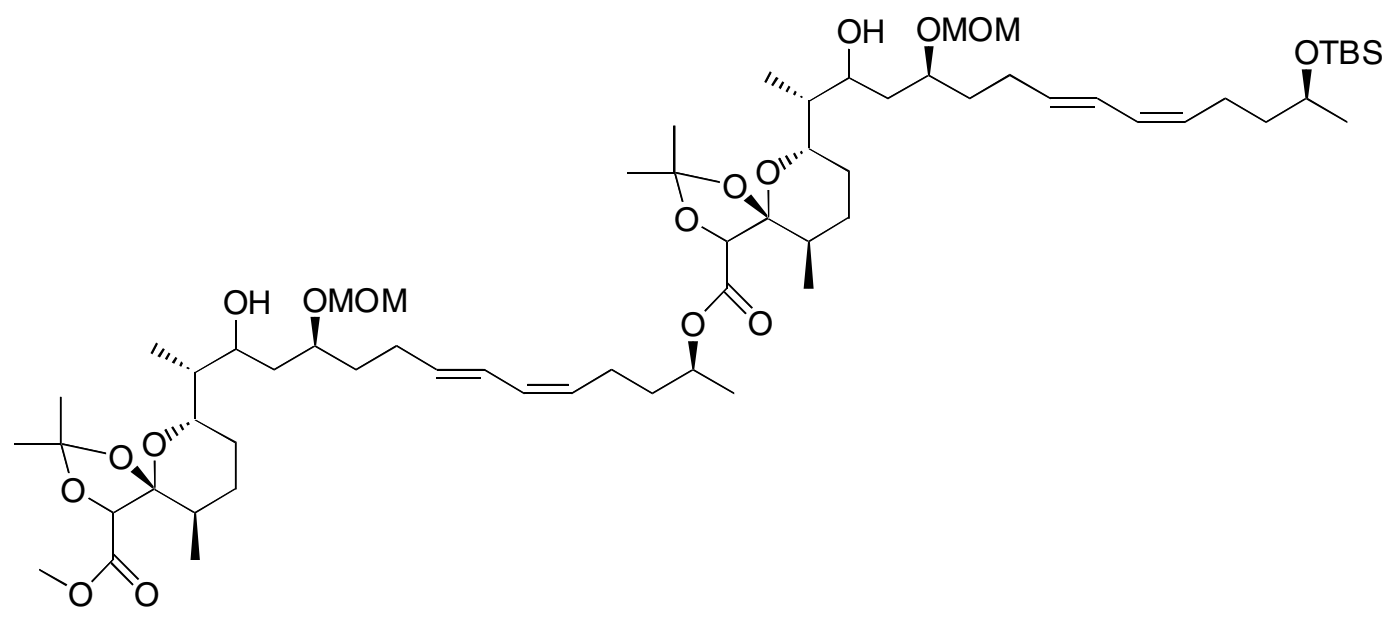

55 (42 mg, 64 $\mu \mathrm{mol}$ ) was dissolved in $\mathrm{MeOH}(2 \mathrm{~mL})$ and fine powdered barium hydroxide octahydrate (400 mg, $1.27 \mathrm{mmol}$ ) was added. The mixture was vigorously stirred for $1 \mathrm{~h}$ at room temperature. The white suspension was diluted with brine $(4 \mathrm{~mL})$ and acidified with $1 \mathrm{M} \mathrm{HCl}$ to $\mathrm{pH} 3$ resulting in a clear solution which was four times extracted with EtOAc. Combined organic layers were washed with brine and dried over $\mathrm{MgSO}_{4}$. The crude acid was judged pure by TLC $\left(\mathrm{R}_{\mathrm{f}}=0.23\right.$ hexane/ EtOAc/MeOH 4:4:1) and used for esterification without further purification. A stock solution was prepared by dissolving 2,4,6trichlorobenzoylchloride $(96 \mathrm{mg}, 400 \mu \mathrm{mol})$ and triethylamine $(44 \mathrm{mg}, 440 \mu \mathrm{mol})$ in $2 \mathrm{~mL}$ toluene. The acid was azeotroped with toluene $(2 \times 0.5 \mathrm{~mL})$ and a portion of the stock solution $(0.48 \mathrm{~mL})$ was added to the acid in toluene $(0.5 \mathrm{~mL})$. After stirring for $1 \mathrm{~h}$, the mixture was diluted with toluene $(0.6 \mathrm{~mL})$ and added over $1 \mathrm{~h}$ via syringe pump to a solution 
of methyl $(5 S, 7 S, 10 R)-2,2,10-$ trimethyl-7-[(1R, 4S, 7E, 9Z, 13S)-2,13-dihydroxy-4methoxymethyloxy-1-methyl-tetradeca-7,9-dien-1-yl]-1,3,6-trioxaspiro[4.5]decan-4-

carboxylate 56b $(38 \mathrm{mg}, 70 \mu \mathrm{mol})$ and DMAP $(12 \mathrm{mg}, 100 \mu \mathrm{mol})$ in $3 \mathrm{~mL}$ toluene at room temperature. The suspension was stirred for an additional $0.5 \mathrm{~h}$, diluted with EtOAc $(10 \mathrm{~mL})$ and saturated aqueous $\mathrm{NaHCO}_{3}(5 \mathrm{~mL})$ were added. The phases were separated and the aqueous layer was extracted three times with EtOAc. The combined organic extracts were washed with brine, dried over $\mathrm{MgSO}_{4}$ and concentrated in vacuo. The residue was purified by column chromatography (25 $\mathrm{g}$ of $\mathrm{SiO}_{2}$, hexanes/ EtOAc $2: 1, \mathrm{R}_{\mathrm{f}}=0.26$ ) to provide $55 \mathrm{mg}$ (74\%) of the protected dimer $\mathbf{5 9}$ as a clear colorless oil and $7 \mathrm{mg}(13 \mu \mathrm{mol})$ of recovered alcohol 56b.

59: Anal. calcd for $\mathrm{C}_{63} \mathrm{H}_{110} \mathrm{O}_{17} \mathrm{Si}$ : C 64.80; H, 9.50; found: C, 64.65; H, 9.20. ${ }^{1} \mathrm{H}-\mathrm{NMR}$ (400 $\left.\mathrm{MHz} ; \mathrm{CDCl}_{3}\right): \delta[\mathrm{ppm}]=6.31(\mathrm{dd}, J=15.1,11.3 \mathrm{~Hz}, 1 \mathrm{H}), 6.27(\mathrm{dd}, J=15.1,11.3 \mathrm{~Hz}, 1 \mathrm{H})$, $5.95(\mathrm{dd}, J=11.3,11.0 \mathrm{~Hz}, 1 \mathrm{H}), 5.92(\mathrm{dd}, J=11.3,11.0 \mathrm{~Hz}, 1 \mathrm{H}), 5.67(\mathrm{dt}, J=15.1,7.1 \mathrm{~Hz}$, $1 \mathrm{H}), 5.63(\mathrm{dt}, J=15.1,7.1 \mathrm{~Hz}, 1 \mathrm{H}), 5.30(\mathrm{dt}, J=11.0,7.4 \mathrm{~Hz}, 1 \mathrm{H}), 5.26(\mathrm{dt}, J=11.0,7.4$ $\mathrm{Hz}, 1 \mathrm{H}), 5.02(\mathrm{qt}, J=6.4,6.2 \mathrm{~Hz}, 1 \mathrm{H}), 4.66(\mathrm{~d}, J=6.7 \mathrm{~Hz}, 1 \mathrm{H}), 4.64(\mathrm{~s}, 2 \mathrm{H}), 4.63(\mathrm{~d}, J=6.7$ Hz, 1H), 4.58 (s, 1H), 4.49 (s, 1H), 4.15 (m, 1H), 3.99 (m, 1H), 3.97-4.07 (m, 1H), 3.76-3.91 (m, 5H), $3.75(\mathrm{~s}, 3 \mathrm{H}), 3.37(\mathrm{~s}, 6 \mathrm{H}), 2.08-2.34(\mathrm{~m}, 8 \mathrm{H}), 1.91-2.01(\mathrm{~m}, 2 \mathrm{H}), 1.73-1.90$ (m, 4H), 1.40-1.72 (m, 18H), $1.62(\mathrm{~s}, 3 \mathrm{H}), 1.52(\mathrm{~s}, 3 \mathrm{H}), 1.51(\mathrm{~s}, 3 \mathrm{H}), 1.40(\mathrm{~s}, 3 \mathrm{H}), 4 \mathrm{H}), 1.27$ (d, $J=6.4 \mathrm{~Hz}, 3 \mathrm{H}), 1.12(\mathrm{~d}, J=6.0 \mathrm{~Hz}, 3 \mathrm{H}), 0.96(\mathrm{~d}, J=6.4 \mathrm{~Hz}, 3 \mathrm{H}), 0.92(\mathrm{~d}, J=7.1 \mathrm{~Hz}, 3 \mathrm{H})$, $0.88(\mathrm{~d}, J=6.9 \mathrm{~Hz}, 3 \mathrm{H}), 0.88(\mathrm{~s}, 9 \mathrm{H}), 0.78(\mathrm{~d}, J=6.4 \mathrm{~Hz}, 1.5 \mathrm{H}), 0.04(\mathrm{~s}, 6 \mathrm{H}) ;{ }^{13} \mathrm{C}-\mathrm{NMR}(63$ $\left.\mathrm{MHz} ; \mathrm{CDCl}_{3}\right): \delta[\mathrm{ppm}]=168.9,168.2,134.8,133.8,130.1,129.3,128.5,128.3,125.9$, $125.5,112.5,110.7,107.6,107.5,95.4,95.3,84.3,80.4,76.2,76.1,75.4,74.5,72.3,68.5$, 68.2, 68.1, 55.7, 52.2, 42.8, 42.3, 39.7, 39.3, 35.6, 34.2, 34.1, 34.0, 29.0, 28.8, 28.5, 28.4, $28.0,26.6,25.9,25.5,24.0,23.7,19.6,18.1,16.2,16.1,10.5,10.1,-4.4,-4.7 ;[\alpha]_{\mathrm{D}}^{20}=$ $+46.0^{\circ}\left(\mathrm{c}=1.0 ; \mathrm{CHCl}_{3}\right) ; \mathrm{MS}:\left(\mathrm{FI}, 7 \mathrm{kV}, 3 \mathrm{~mA}, 270^{\circ} \mathrm{C}\right) \mathrm{m} / \mathrm{z}=1167$ [M] (100), 1022 (6), 846 (18); IR (thin film): $1 / \lambda\left[\mathrm{cm}^{-1}\right]=3524,2933,1765,1455,1381,1216,1121,1035,919,836$, 774.

\section{Dimeric triol (60)}




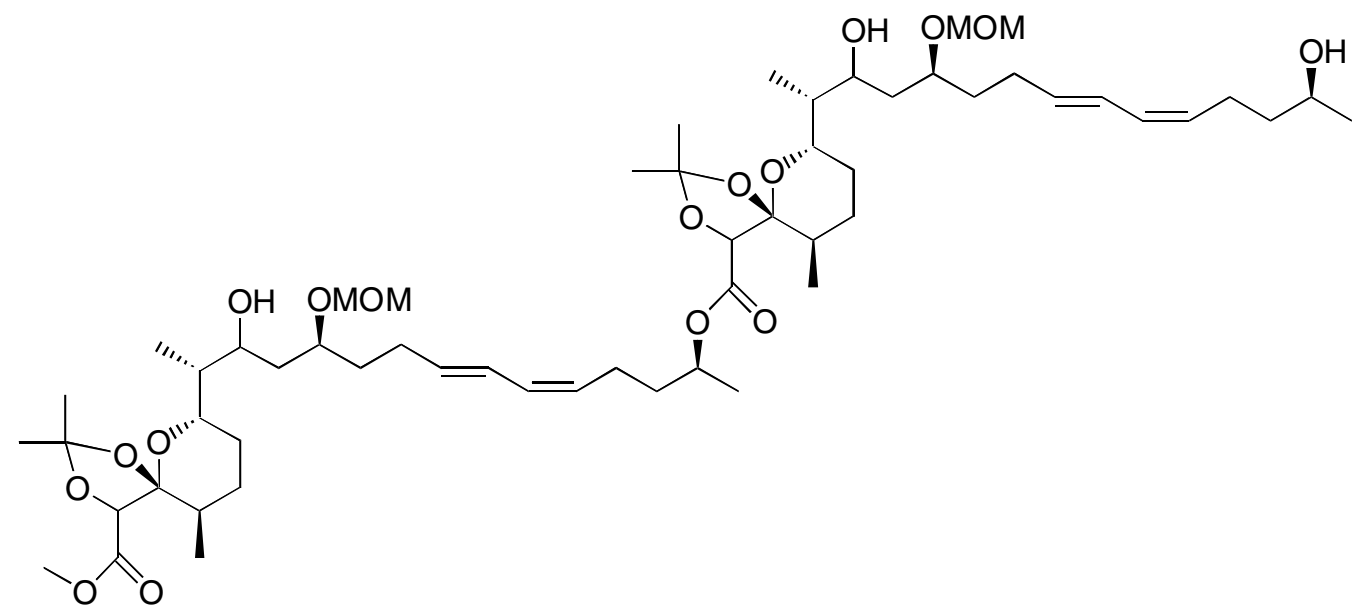

A stock solution of $5 \mathrm{~mL} \mathrm{HF} /$ pyridine complex, $10 \mathrm{~mL}$ pyridine and $15 \mathrm{~mL}$ THF was prepared. In a Teflon vessel the protected dimer 59 (51 $\mathrm{mg}, 43,7 \mu \mathrm{mol})$ was dissolved in 3 $\mathrm{mL}$ THF and $0.5 \mathrm{~mL}$ of the stock solution was added slowly at ambient temperature. The mixture was stirred for $24 \mathrm{~h}$ and then diluted with $\mathrm{Et}_{2} \mathrm{O}(15 \mathrm{~mL})$ and saturated aqueous $\mathrm{CuSO}_{4}(10 \mathrm{~mL})$. The phases were separated and aqueous layer was extracted three times with EtOAc. The combined organic layers were washed with saturated aqueous $\mathrm{NaHCO}_{3}$, dried over $\mathrm{MgSO}_{4}$ and concentrated in vacuo. The residue was purified by column chromatography $\left(25 \mathrm{~g} \mathrm{SiO}_{2}, 50 \%\right.$ EtOAc/hexanes, $\left(\mathrm{R}_{\mathrm{f}}=0.14\right)$ to provide $44 \mathrm{mg}(96 \%)$ of dimeric triol $\mathbf{6 0}$ as a clear colorless oil. ${ }^{1} \mathrm{H}-\mathrm{NMR}\left(600 \mathrm{MHz} ; \mathrm{CDCl}_{3}\right): \delta[\mathrm{ppm}]=6.34(\mathrm{dd}, J=15.1,11.0 \mathrm{~Hz}, 1 \mathrm{H})$, $6.26(\mathrm{dd}, J=15.1,10.9 \mathrm{~Hz}, 1 \mathrm{H}), 5.95(\mathrm{dd}, J=11.0,10.8 \mathrm{~Hz} 1 \mathrm{H}), 5.94(\mathrm{dd}, J=10.9,10.6$ $\mathrm{Hz}, 1 \mathrm{H}), 5.66(\mathrm{dt}, J=15.1,7.0 \mathrm{~Hz}, 1 \mathrm{H}), 5.65(\mathrm{dt}, J=15.1,6.9 \mathrm{~Hz}, 1 \mathrm{H}), 5.30(\mathrm{dt}, J=10.8$, $7.6 \mathrm{~Hz}, 1 \mathrm{H}), 5.26(\mathrm{dt}, J=10.6,7.6 \mathrm{~Hz}, 1 \mathrm{H}), 5.02(\mathrm{qt}, J=6.4,6.2 \mathrm{~Hz}, 1 \mathrm{H}), 4.66(\mathrm{~d}, J=6.8$ Hz, 1H), 4.64 (s, 2H), 4.63 (d, $J=6.8 \mathrm{~Hz}, 1 \mathrm{H}), 4.58$ (s, 1H), 4.49 (s, 1H), 4.14 (ddd, $J=9.0$, 3.7, $2.2 \mathrm{~Hz}, 1 \mathrm{H}), 3.99(\mathrm{~m}, 1 \mathrm{H}), 3.87$ (ddd, $J=11.3,6.1,2.1 \mathrm{~Hz}, 1 \mathrm{H}), 3.82$ (ddd, $J=11.5$, 5.5, $2.2 \mathrm{~Hz}, 1 \mathrm{H}), 3.73-3.82(\mathrm{~m}, 3 \mathrm{H}), 3.74(\mathrm{~s}, 3 \mathrm{H}), 3.36(\mathrm{~s}, 6 \mathrm{H}), 2.12-2.28(\mathrm{~m}, 8 \mathrm{H}), 1.97$ (dqd, $J=10.9,6.5,4.1 \mathrm{~Hz}, 1 \mathrm{H}), 1.85$, (dqd $J=10.9,6.4,4.2 \mathrm{~Hz}, 1 \mathrm{H}), 1.73-1.83(\mathrm{~m}, 4 \mathrm{H})$, 1.40-1.72 (m, 18H), 1.62 (s, 3H), 1.52 (s, 3H), 1.51 (s, 3H), 1.40 (s, 3H), 1.27 (d, J = 6.3 Hz, $3 \mathrm{H}), 1.18(\mathrm{~d}, J=6.3 \mathrm{~Hz}, 3 \mathrm{H}), 0.96(\mathrm{~d}, J=6.5 \mathrm{~Hz}, 3 \mathrm{H}), 0.92(\mathrm{~d}, J=7.0 \mathrm{~Hz}, 3 \mathrm{H}), 0.87$ (d, $J=$ $7.0 \mathrm{~Hz}, 3 \mathrm{H}), 0.77(\mathrm{~d}, J=6.4 \mathrm{~Hz}, 3 \mathrm{H}) ;{ }^{13} \mathrm{C}-\mathrm{NMR}\left(63 \mathrm{MHz} ; \mathrm{CDCl}_{3}\right): \delta[\mathrm{ppm}]=168.9,168.2$, $134.8,134.2,129.5,129.3,129.0,128.2,125.8,125.5,112.5,110.7,107.6,107.5,95.4$, 84.3, 80.4, 76.1, 75.4, 74.5, 72.3, 68.4, 68.2, 64.4, 55.7, 52.2, 42.8, 42.3, 39.3, 39.2, 39.0, 35.6, 34.2, 33.9, 29.0, 28.8, 28.5, 28.4, 28.0, 26.9, 26.6, 25.5, 24.1, 23.7, 23.6, 19.5, 16.1, $10.5,10.1 ;[\alpha]_{\mathrm{D}}^{20}=+29.8^{\circ}\left(\mathrm{c}=0.5 ; \mathrm{CHCl}_{3}\right) ; \mathrm{MS}:\left(\mathrm{FI}, 7 \mathrm{kV}, 3 \mathrm{~mA}, 255^{\circ} \mathrm{C}\right) \mathrm{m} / \mathrm{z}=1054[\mathrm{M}]$ 


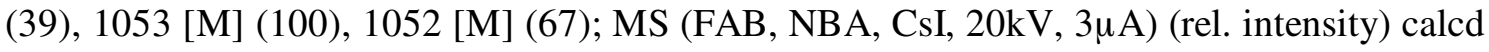
for $\mathrm{C}_{57} \mathrm{H}_{96} \mathrm{O}_{17} \mathrm{Cs} 1185.6$ (100), 1186.6 (66), 1187.6 (25), 1188.6 (7), found : 1185.2 (100), 1186.2 (56), 1187.3 (22), 1156.3 (6); HRMS (FAB) calcd for $\mathrm{C}_{57} \mathrm{H}_{96} \mathrm{O}_{17} \mathrm{Cs}$ : [M+Cs] :1185.5702 found: 1185.5695 ; IR (thin film): $1 / \lambda\left[\mathrm{cm}^{-1}\right]=3502,2936,1760,1456,1381$, 1216, 1120, 1033, 920, 836 .

\section{Cyclic dimeric diole (62)}

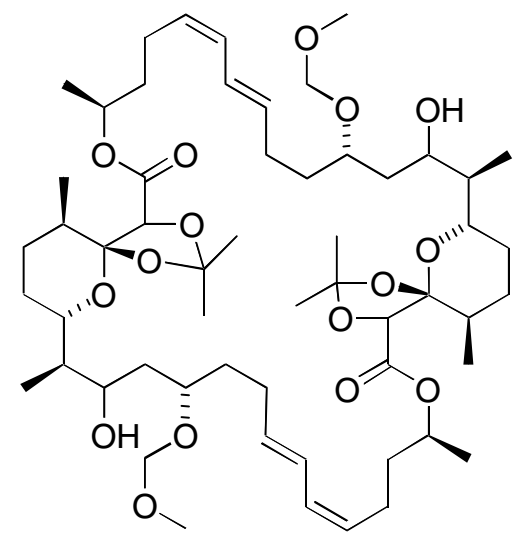

Dimeric triol $60(10.5 \mathrm{mg}, 10 \mu \mathrm{mol})$ was dissolved in $\mathrm{MeOH}(1.3 \mathrm{~mL})$ and powdered $\mathrm{Ba}(\mathrm{OH})_{2} 8 \mathrm{H}_{2} \mathrm{O}(200 \mathrm{mg}, 0.63 \mathrm{mmol})$ was added. The white suspension was vigorously stirred

for $30 \mathrm{~min}$, then diluted with $5 \mathrm{~mL} \mathrm{Et}_{2} \mathrm{O}$ and $5 \mathrm{~mL}$ brine and acidified to $\mathrm{pH} 3$ with $1 \mathrm{M} \mathrm{HCl}$ (1.4 mL). The two clear phases were separated and the aqueous layer was extracted four times with EtOAc. The combined organic layers were washed with brine, dried over $\mathrm{MgSO}_{4}$ and concentrated in vacuo. The crude dimeric seco acid $\mathbf{6 1}$ was used for macrolactonization without any further purification. A stock solution was prepared by dissolving 2,4,6trichlorobenzoylchloride $(96 \mathrm{mg}, 400 \mu \mathrm{mol})$ and triethylamine $(44 \mathrm{mg}, 440 \mu \mathrm{mol})$ in $2 \mathrm{~mL}$ toluene. A portion of $0.1 \mathrm{ml}$ was added to the dimeric seco acid in $0.5 \mathrm{~mL}$ toluene. After stirring for $2 \mathrm{~h}$ the mixture was diluted with $0.6 \mathrm{~mL}$ toluene and added over $2 \mathrm{~h}$ via syringe pump to a solution of DMAP $(12 \mathrm{mg}, 100 \mu \mathrm{mol})$ in $3 \mathrm{~mL}$ toluene at $35^{\circ} \mathrm{C}$. The suspension was stirred for an additional $0.5 \mathrm{~h}$ diluted with EtOAc $(10 \mathrm{~mL})$ and saturated aqueous $\mathrm{NaHCO}_{3}(5 \mathrm{~mL})$. The phases were separated and the aqueous layer was extracted three times with EtOAc. The combined organic layers were washed with brine, dried over $\mathrm{MgSO}_{4}$ and 
concentrated in vacuo. The residue was purified by column chromatography $\left(12 \mathrm{~g} \mathrm{SiO}_{2}, 50 \%\right.$ EtOAc/hexanes; $\left.\mathrm{R}_{\mathrm{f}}=0.41\right)$ to provide $8.4 \mathrm{mg}(82 \%)$ of $\mathbf{6 2}$ as a clear colorless oil.

${ }^{1} \mathrm{H}-\mathrm{NMR}\left(600 \mathrm{MHz} ; \mathrm{CDCl}_{3}\right): \delta[\mathrm{ppm}]=6.30(\mathrm{dd}, J=15.4,11.0 \mathrm{~Hz}, 2 \mathrm{H}), 5.97(\mathrm{dd}, J=11.0$, $10.8 \mathrm{~Hz}, 1 \mathrm{H}), 5.95(\mathrm{dd}, J=11.0,10.8 \mathrm{~Hz}, 1 \mathrm{H}), 5.71(\mathrm{dt}, J=15.4,6.9 \mathrm{~Hz}, 1 \mathrm{H}), 5.68(\mathrm{dt}, J=$ 15.4, 7.0 Hz, 1H), $5.28(\mathrm{dt}, J=10.8,7.5 \mathrm{~Hz}, 2 \mathrm{H}), 4.96-5.06(\mathrm{~m}, 2 \mathrm{H}), 4.68(\mathrm{~d}, J=6.7 \mathrm{~Hz}$, $1 \mathrm{H}), 4.66(\mathrm{~s}, 2 \mathrm{H}), 4.63(\mathrm{~d}, J=6.7 \mathrm{~Hz}, 1 \mathrm{H}), 4.54(\mathrm{~s}, 1 \mathrm{H}), 4.50(\mathrm{~s}, 1 \mathrm{H}), 4.16(\mathrm{~m}, 1 \mathrm{H}), 4.07$ (m, $1 \mathrm{H}), 3.86-3.91(\mathrm{~m}, 1 \mathrm{H}), 3.76-3.82(\mathrm{~m}, 3 \mathrm{H}), 3.38$ (s, 6H), 2.12-2.29 (m, 8H), 1.94-1.99 (m, 1H), 1.89, (ddd, $J=14.4,10.0,5.1 \mathrm{~Hz}, 1 \mathrm{H}), 1.73-1.84(\mathrm{~m}, 4 \mathrm{H}), 1.50-1.72$ (m, 14H), 1.63 (s, $3 \mathrm{H}), 1.54(\mathrm{~s}, 3 \mathrm{H}), 1.51(\mathrm{~s}, 3 \mathrm{H}), 1.40(\mathrm{~s}, 3 \mathrm{H}), 1.32-1.38(\mathrm{~m}, 4 \mathrm{H}) 1.29(\mathrm{~d}, J=6.1 \mathrm{~Hz}, 3 \mathrm{H})$, $1.23(\mathrm{~d}, J=6.2 \mathrm{~Hz}, 3 \mathrm{H}), 1.00(\mathrm{~d}, J=7.2 \mathrm{~Hz}, 3 \mathrm{H}), 0.97$ (d, $J=6.4 \mathrm{~Hz}, 3 \mathrm{H}), 0.87$ (d, $J=6.9$ $\mathrm{Hz}, 3 \mathrm{H}), 0.81(\mathrm{~d}, J=6.7 \mathrm{~Hz}, 3 \mathrm{H}) ;{ }^{13} \mathrm{C}-\mathrm{NMR}\left(63 \mathrm{MHz} ; \mathrm{CDCl}_{3}\right): \delta[\mathrm{ppm}]=168.8,134.8$, 134.7, 129.4, 129.2, 128.6, 128.2, 125.5, 112.3, 110.6, 107.3, 107.2, 95.5, 95.4, 84.1, 80.2, 76.6, 75.9, 73.5, 72.3, 71.5, 67.7, 67.6, 64.4, 55.7, 43.8, 42.3, 39.8, 39.6, 35.9, 35.6, 34.6, 34.4, 33.9, 33.8, 29.5, 28.9, 28.5, 28.0, 27.0, 26.4, 25.2, 23.9, 23.7, 19.7, 16.2, 16.0, 10.5,

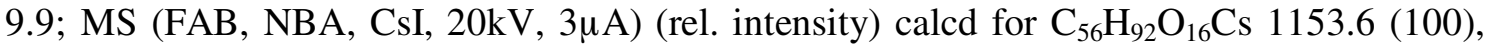
1154.6 (65), 1155.6 (24), 1156.6 (6), found : 1153.6 (100), 1154.6 (62), 1155.6 (24), 1156.6 (7); HRMS (FAB) calcd for $\mathrm{C}_{56} \mathrm{H}_{92} \mathrm{O}_{16} \mathrm{Cs}:[\mathrm{M}+\mathrm{Cs}]: 1153.5439$ found: 1153.5206 ; $[\alpha]_{\mathrm{D}}^{20}=$ $+44.2^{\circ}\left(\mathrm{c}=1.0 ; \mathrm{CHCl}_{3}\right) ; \mathrm{IR}$ (thin film): $1 / \lambda\left[\mathrm{cm}^{-1}\right]=3545,2935,1757,1729,1458,1381$; 1274, 1217, 1119, 1036, 955, 736.

\section{MOM/acetonide protected tartrolon A 2 (63)}

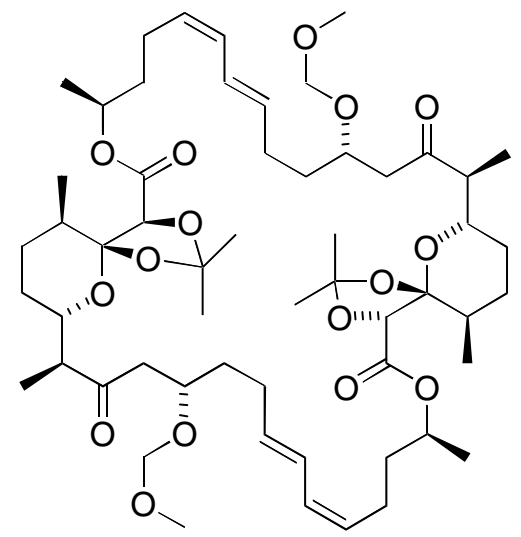

$4(35 \mathrm{mg}, 34 \mu \mathrm{mol})$ in $\mathrm{CH}_{2} \mathrm{Cl}_{2}(2 \mathrm{~mL})$ was oxidized under Swern conditions (TP 1) to give, after column chromatography $\left(15 \mathrm{~g}\right.$ of $\mathrm{SiO}_{2}$, EtOAc/hexanes $\left.1: 1, \mathrm{R}_{\mathrm{f}}=0.63\right)$ to provide 31 
mg (89\%) of MOM/acetonide protected tartrolon A $2 \mathbf{6 3}$ as a colorless foam. ${ }^{1} \mathrm{H}-\mathrm{NMR}$ (400 $\left.\mathrm{MHz} ; \mathrm{CDCl}_{3}\right): \delta[\mathrm{ppm}]=6.46(\mathrm{dd}, J=15.0,11.2 \mathrm{~Hz}, 1 \mathrm{H}), 6.28(\mathrm{dd}, J=15.0,11.2 \mathrm{~Hz}, 1 \mathrm{H})$, $6.00(\mathrm{dd}, J=11.2,11.0 \mathrm{~Hz}, 1 \mathrm{H}), 5.96(\mathrm{dd}, J=11.2,11.0 \mathrm{~Hz}, 1 \mathrm{H}), 5.76(\mathrm{dt}, J=15.0,6.5 \mathrm{~Hz}$, $1 \mathrm{H}), 5.66(\mathrm{dt}, J=15.0,7.0 \mathrm{~Hz}, 0.5 \mathrm{H}), 5.30(\mathrm{dt}, J=11.0,7.4 \mathrm{~Hz}, 1 \mathrm{H}), 5.28(\mathrm{dt}, J=11.0,7.4$ $\mathrm{Hz}, 1 \mathrm{H}), 5.04-5.14(\mathrm{~m}, 2 \mathrm{H}), 4.74(\mathrm{~d}, J=7.0 \mathrm{~Hz}, 1 \mathrm{H}), 4.71(\mathrm{~d}, J=7.0 \mathrm{~Hz}, 1 \mathrm{H}), 4.68(\mathrm{~d}, J=$ $6.0 \mathrm{~Hz}, 1 \mathrm{H}), 4.62$ (d, J = 6.0 Hz, 1H), 4.49 (s, 1H), 4.39 (s, 1H), 4.05-4.15 (m, 2H), 3.93 (ddd, $J=11.5,8.7,2.0 \mathrm{~Hz}, 1 \mathrm{H}), 3.86$ (ddd, $J=11.5,8.7,2.0 \mathrm{~Hz}, 0.5 \mathrm{H}), 3.37$ (s, 3H), 3.35 (s, $3 \mathrm{H}), 2.86(\mathrm{dd}, J=17.5,7.7 \mathrm{~Hz}, 1 \mathrm{H}) 2.62-2.74(\mathrm{~m}, 4 \mathrm{H}), 2.51(\mathrm{dq}, J=9.5,7.0 \mathrm{~Hz}, 1 \mathrm{H}), 2.12-$ $2.32(\mathrm{~m}, 4 \mathrm{H}), 2.03(\mathrm{dqd}, J=10.5,7.0,5.7 \mathrm{~Hz}, 1 \mathrm{H}), 1.89(\mathrm{dqd}, J=11.0,6.5,3.7 \mathrm{~Hz}, 1 \mathrm{H})$, $1.58-1.82(\mathrm{~m}, 8 \mathrm{H}), 1.57(\mathrm{~s}, 3 \mathrm{H}), 1.51(\mathrm{~s}, 3 \mathrm{H}), 1.42(\mathrm{~s}, 3 \mathrm{H}), 1.38(\mathrm{~s}, 3 \mathrm{H}), 1.25-1.45(\mathrm{~m}, 8 \mathrm{H})$, $1.34(\mathrm{~d}, J=6.5 \mathrm{~Hz}, 3 \mathrm{H}), 1.30(\mathrm{~d}, J=6.4 \mathrm{~Hz}, 3 \mathrm{H}), 1.00(\mathrm{~d}, J=7.0 \mathrm{~Hz}, 3 \mathrm{H}), 0.99$ (d, $J=7.0$ $\mathrm{Hz}, 3 \mathrm{H}), 0.94(\mathrm{~d}, J=7.0 \mathrm{~Hz}, 3 \mathrm{H}), 0.77(\mathrm{~d}, J=6.5 \mathrm{~Hz}, 3 \mathrm{H}) ;{ }^{13} \mathrm{C}-\mathrm{NMR}\left(63 \mathrm{MHz} ; \mathrm{CDCl}_{3}\right): \delta$ $[\mathrm{ppm}]=213.0,212.1,168.8,167.4,135.3,134.7,130.0,129.5,129.0,128.4,126.0,125.9$, 112.7, 111.1, 107.9, 107.1, 96.8, 84.5, 79.9, 75.5, 74.8, 73.9, 73.8, 72.5, 71.5, 56.0, 55.9, 51.9, 51.7, 51.2, 50.4, 36.5, 36.1, 35.0, 34.7, 30.1, 29.5, 29.3, 29.0, 28.8, 28.1, 27.3, 27.1, 25.7, 24.4, 23.9, 20.7, 19.8, 16.6, 16.4, 13.0, 12.7; MS (FAB, NBA, CsI, 20 kV, $3 \mu \mathrm{A}$ ) (rel. intensity) calcd for $\mathrm{C}_{56} \mathrm{H}_{88} \mathrm{O}_{16} \mathrm{Cs} 1149.6$ (100), 1150.6 (65), 1151.6 (24), 1152.6 (6), found : 1149.6 (100), 1150.6 (61), 1151.6 (36), 1152.6 (14); HRMS (FAB) calcd. for $\mathrm{C}_{56} \mathrm{H}_{88} \mathrm{O}_{16} \mathrm{Cs}$ : $[\mathrm{M}+\mathrm{Cs}]: 1149.5127$ found : 1149.5058; (EI, $\left.80 \mathrm{eV}, 190^{\circ} \mathrm{C}\right)$ calcd. for $\mathrm{C}_{54} \mathrm{H}_{81} \mathrm{O}_{14}[\mathrm{M}-$ $\left.\mathrm{C}_{2} \mathrm{H}_{7} \mathrm{O}_{2}\right]: 953.5626$ found : $953.5689 .[\alpha]_{\mathrm{D}}^{20}=+55.0^{\circ}\left(\mathrm{c}=0.67 ; \mathrm{CHCl}_{3}\right)$. IR (thin film): $1 / \lambda$ $\left[\mathrm{cm}^{-1}\right]=2937,1758,1719,1458,1381,1215,1117,1044$.

\section{Tartrolon B (1)}

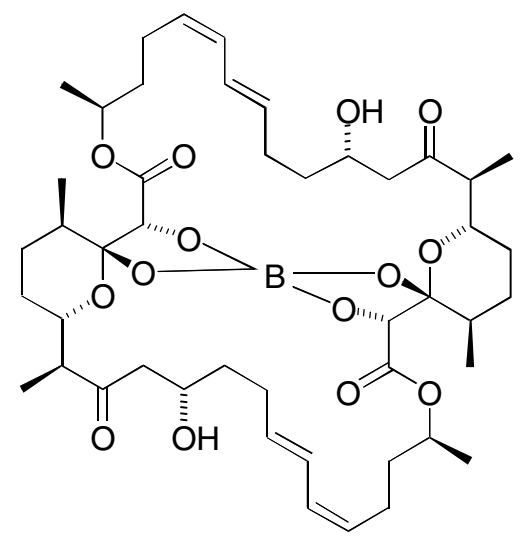


To a stirred solution of $\mathbf{6 3}(10.3 \mathrm{mg}, 10 \mu \mathrm{mol})$ in $\mathrm{CH}_{2} \mathrm{Cl}_{2}(2 \mathrm{~mL})$ at $-78^{\circ} \mathrm{C}$ was added a 0.5 $\mathrm{M}$ solution of $\mathrm{NEt}_{3}(0.1 \mathrm{ml}, 0.05 \mathrm{mmol})$ and a $1 \mathrm{M}$ solution of dimethylboron bromide $(0.3$ $\mathrm{mL}, 0.3 \mathrm{mmol}$ ) in $\mathrm{CH}_{2} \mathrm{Cl}_{2}$. After $4 \mathrm{~h}$ at $-78^{\circ} \mathrm{C}, 3 \mathrm{~mL}$ water and $5 \mathrm{~mL}$ THF were added and the mixture was quickly warmed to ambient temperature. After 10 min of efficient stirring $3 \mathrm{~mL}$ brine and $10 \mathrm{~mL}$ EtOAc were added. The phases were separated and the aqueous layer was extracted three times with EtOAc. The combined organic layers were washed with brine, dried over $\mathrm{MgSO}_{4}$ and concentrated in vacuo. The residue was filtered though a short column (10 $\mathrm{g} \mathrm{SiO}_{2} ; 10 \% \mathrm{MeOH}$ in $\mathrm{CH}_{2} \mathrm{Cl}_{2}$ ) to provide $5.5 \mathrm{mg}(65 \%)$ of a crude mixture of all three tartrolon $\mathrm{A}$ forms. The unseparated mixture was dissolved in $\mathrm{MeOH}(2 \mathrm{~mL}) . \mathrm{Na}_{2} \mathrm{~B}_{4} \mathrm{O}_{7} 10$ $\mathrm{H}_{2} \mathrm{O}(5 \mathrm{mg}, 12 \mu \mathrm{mol})$ was added and the mixture was warmed to $55^{\circ} \mathrm{C}$ for $12 \mathrm{~h}$. The cold mixture was concentrated under reduced pressure and dissolved in $\mathrm{CH}_{2} \mathrm{Cl}_{2}(2 \mathrm{~mL})$. The solution was passed through a Sephatex ${ }^{\circledR} \mathrm{LH} 20$ column $\left(10 \mathrm{~g}, \mathrm{CH}_{2} \mathrm{Cl}_{2} / \mathrm{MeOH} 10\right.$ : 1). The residue was purified by HPLC (data for Merck Supersphere Si $604 \mu \mathrm{m}, 244 \mathrm{~mm}$ x $4 \mathrm{~mm}$ column, $25 \%$ EtOAc in hexane, flow: $\left.2 \mathrm{~mL} / \mathrm{min}, \mathrm{t}_{\mathrm{R}}=2.2 \mathrm{~min}\right)$ to provide $2.3 \mathrm{mg}(27 \%$ over two steps) tartrolon B 1 as a colorless foam. ${ }^{1} \mathrm{H}-\mathrm{NMR}\left(600 \mathrm{MHz} ; \mathrm{CDCl}_{3}\right): \delta[\mathrm{ppm}]=6.07$ (dd, $J=10.8,10.4 \mathrm{~Hz}, 2 \mathrm{H}), 5.98(\mathrm{dd}, J=15.5,10.8 \mathrm{~Hz}, 2 \mathrm{H}), 5.91(\mathrm{dt}, J=15.5,4.2 \mathrm{~Hz}, 2 \mathrm{H})$, $5.26(\mathrm{ddd}, J=10.8,10.8,6.0 \mathrm{~Hz}, 2 \mathrm{H}), 4.65(\mathrm{dq}, J=10.4,6.0 \mathrm{~Hz}, 2 \mathrm{H}), 4.43$ (s, 2H), 4.01 (ddd, $J=10.8,10.8,1.3 \mathrm{~Hz}, 2 \mathrm{H}), 3.98(\mathrm{~m}, 2 \mathrm{H}), 3.33(\mathrm{~s}, 2 \mathrm{H}), 3.22(\mathrm{dd}, J=19.0,10.3 \mathrm{~Hz}$, 2H), 2.36-2.46 (m, 4H), 2.38 (d, $J=19.0 \mathrm{~Hz}, 2 \mathrm{H}), 2.21(\mathrm{dq}, J=10.8,7.3 \mathrm{~Hz}, 2 \mathrm{H}), 1.89-2.02$ (m, 4H), 1.74-1.86 (m, 6H), 1.50-1.73 (m, 6H), 1.34 (m, 2H), $1.26(\mathrm{~m}, 2 \mathrm{H}), 1.19(\mathrm{~d}, J=6.5$ $\mathrm{Hz}, 6 \mathrm{H}), 1.06(\mathrm{~m}, 2 \mathrm{H}), 1.0$ (d, $J=6.0 \mathrm{~Hz}, 6 \mathrm{H}), 0.99$ (d, $J=6.5 \mathrm{~Hz}, 6 \mathrm{H}) ;{ }^{13} \mathrm{C}-\mathrm{NMR}(63 \mathrm{MHz}$; $\left.\mathrm{CDCl}_{3}\right): \delta[\mathrm{ppm}]=214.8,174.4,134.7,131.0,127.8,122.9,104.4,78.2$, 71.2, 69.4, 67.7, $54.9,42.5,35.9,35.7,31.6,28.3,28.2,23.0,20.6,16.9,12.8 ; \mathrm{MS}(\mathrm{FAB}, \mathrm{NBA}): \mathrm{m} / \mathrm{z}=878$ $[\mathrm{M}], 855[\mathrm{M}-\mathrm{Na}], \mathrm{UV}: \lambda_{\max }=235 \mathrm{~nm}(\varepsilon=19400 ; \mathrm{MeOH}) . \quad[\alpha]_{\mathrm{D}}^{20}=+27.0^{\circ}\left(\mathrm{c}=0.1, \mathrm{CHCl}_{3}\right)$, ref. $^{3 \mathrm{a}}[\alpha]_{\mathrm{D}}^{20}=+28.7^{\circ}\left(\mathrm{c}=1, \mathrm{CHCl}_{3}\right) . \mathrm{CD}: \lambda_{\max }=205 \mathrm{~nm}(\Delta \varepsilon=-4.5 ; \mathrm{MeOH}) ;$ natural sample $\left((\Delta \varepsilon=-7.5)\right.$; IR: (thin film): $1 / \lambda\left[\mathrm{cm}^{-1}\right]=3545,2966,2937,2894,1729,1702,1459,1458$, 1380, 1274, 1119, 1036. 


\section{Comparison of ${ }^{1} \mathrm{H}$-NMR data}

\begin{tabular}{|c|c|c|c|c|}
\hline & \multicolumn{2}{|c|}{ natural tartrolon $\mathrm{B}$} & \multicolumn{2}{|c|}{ synthetic tartrolon B } \\
\hline & $\delta[\mathrm{ppm}]$ & $J[\mathrm{~Hz}]$ & $\delta[\mathrm{ppm}]$ & $J[\mathrm{~Hz}]$ \\
\hline H16 & 6.06 & $\mathrm{dd}, 10.6,10.6$ & 6.07 & $\mathrm{dd}, 10.8,10.4$ \\
\hline H15 & 5.98 & ddd, $15.5,10.6$ & 5.98 & $\mathrm{dd}, 15.5,10.8$ \\
\hline H14 & 5.90 & ddd, $15.5,4.5,4.3$ & 5.91 & $\mathrm{ddd}, 15.5,4.2,4.2$ \\
\hline H17 & 5.26 & ddd, $11.0,10.6,5.5$ & 5.25 & ddd, $10.8,10.8,6.0$ \\
\hline $\mathrm{H} 20$ & 4.64 & dqd, 10.4, 6.2, 1.0 & 4.65 & $\mathrm{dq}, 10.4,6.0$ \\
\hline $\mathrm{H} 2$ & 4.42 & $\mathrm{~s}$ & 4.43 & $\mathrm{~s}$ \\
\hline $\mathrm{H} 7$ & 4.00 & ddd, 11.0, 10.9,1.8 & 4.01 & ddd, $10.8,10.8,1.3$ \\
\hline H11 & 3.97 & ddd, $10.5,10.5,3.2$ & 3.98 & $\mathrm{~m}$ \\
\hline $11 \mathrm{OH}$ & 3.30 & $\mathrm{~s}$ & 3.33 & $\mathrm{~s}$ \\
\hline H10a & 3.22 & $\mathrm{dd}, 19.0,10.5$ & 3.22 & $\mathrm{dd}, 19.0,10.3$ \\
\hline $\mathrm{H} 13 \mathrm{a}$ & 2.41 & $\mathrm{~m}$ & 2.41 & $\mathrm{~m}$ \\
\hline $\mathrm{H} 18 \mathrm{a}$ & 2.41 & $\mathrm{~m}$ & 2.41 & $\mathrm{~m}$ \\
\hline $\mathrm{H} 10 \mathrm{~b}$ & 2.36 & $\mathrm{~d}, 19.0$ & 2.38 & 19.0 \\
\hline $\mathrm{H} 8$ & 2.20 & $\mathrm{dq}, 10.9,7.1$ & 2.21 & $\mathrm{dq}, 10.8,7.3$ \\
\hline H13b & 1.96 & $\mathrm{~m}$ & 1.98 & $\mathrm{~m}$ \\
\hline $\mathrm{H} 18 \mathrm{~b}$ & 1.91 & $\mathrm{~m}$ & 1.93 & $\mathrm{~m}$ \\
\hline $\mathrm{H} 4$ & 1.80 & $\operatorname{ddd}(\mathrm{dqd}), 12.0,6.4,4.5$ & 1.81 & $\mathrm{~m}$ \\
\hline $\mathrm{H} 19 \mathrm{a}$ & 1.80 & $\mathrm{~m}$ & 1.80 & $\mathrm{~m}$ \\
\hline H6a & 1.74 & $\mathrm{~m}$ & 1.76 & $\mathrm{~m}$ \\
\hline $\mathrm{H} 12 \mathrm{a}$ & 1.68 & $\mathrm{~m}$ & 1.68 & $\mathrm{~m}$ \\
\hline H5a & 1.62 & $\mathrm{~m}$ & 1.65 & $\mathrm{~m}$ \\
\hline $\mathrm{H} 5 \mathrm{~b}$ & 1.55 & $\mathrm{~m}$ & $(1.55)$ & $\mathrm{m}$ \\
\hline H19b & 1.32 & $\mathrm{~m}$ & 1.34 & $\mathrm{~m}$ \\
\hline $\mathrm{H} 12 \mathrm{~b}$ & 1.26 & $\mathrm{~m}$ & 1.26 & $\mathrm{~m}$ \\
\hline $\mathrm{H} 21$ & 1.16 & $\mathrm{~d}, 6.2$ & 1.19 & $\mathrm{~d}, 6.5$ \\
\hline H6b & 1.05 & $\mathrm{~m}$ & 1.06 & $\mathrm{~m}$ \\
\hline
\end{tabular}




\begin{tabular}{|c|c|c|c|c|}
\hline $\mathrm{H} 22$ & 1.00 & $\mathrm{~d}, 6.4$ & 1.00 & $\mathrm{~d}, 6.1$ \\
\hline $\mathrm{H} 23$ & 0.99 & $\mathrm{~d}, 7.1$ & 0.99 & $\mathrm{~d}, 6.7$ \\
\hline
\end{tabular}

\section{Comparison of ${ }^{13} \mathrm{C}$-NMR-data}

\begin{tabular}{|c|c|c|}
\hline carbon & natural tartrolon B [ppm] & synthetic tartrolon B [ppm] \\
\hline 9 & 214.8 & 214.8 \\
\hline 1 & 174.4 & 174.4 \\
\hline 14 & 134.7 & 134.7 \\
\hline 17 & 127.8 & 127.8 \\
\hline 15 & 122.9 & 122.9 \\
\hline 16 & 131.0 & 131.0 \\
\hline 3 & 104.4 & 104.4 \\
\hline 2 & 78.2 & 78.2 \\
\hline 11 & 67.8 & 67.7 \\
\hline 7 & 71.3 & 71.2 \\
\hline 20 & 69.5 & 69.4 \\
\hline 8 & 55.0 & 54.9 \\
\hline 10 & 42.6 & 42.5 \\
\hline 19 & 35.9 & 35.9 \\
\hline 4 & 35.7 & 35.7 \\
\hline 12 & 31.7 & 31.6 \\
\hline 6 & 30.4 & 30.3 \\
\hline 13 & 28.3 & 28.3 \\
\hline 5 & 28.2 & 28.2 \\
\hline 21 & 20.6 & 20.6 \\
\hline 22 & 16.9 & 16.9 \\
\hline 18 & 23.0 & 23.0 \\
\hline
\end{tabular}


Figure. Crystal structure of tartrolon B (potassium derivative)

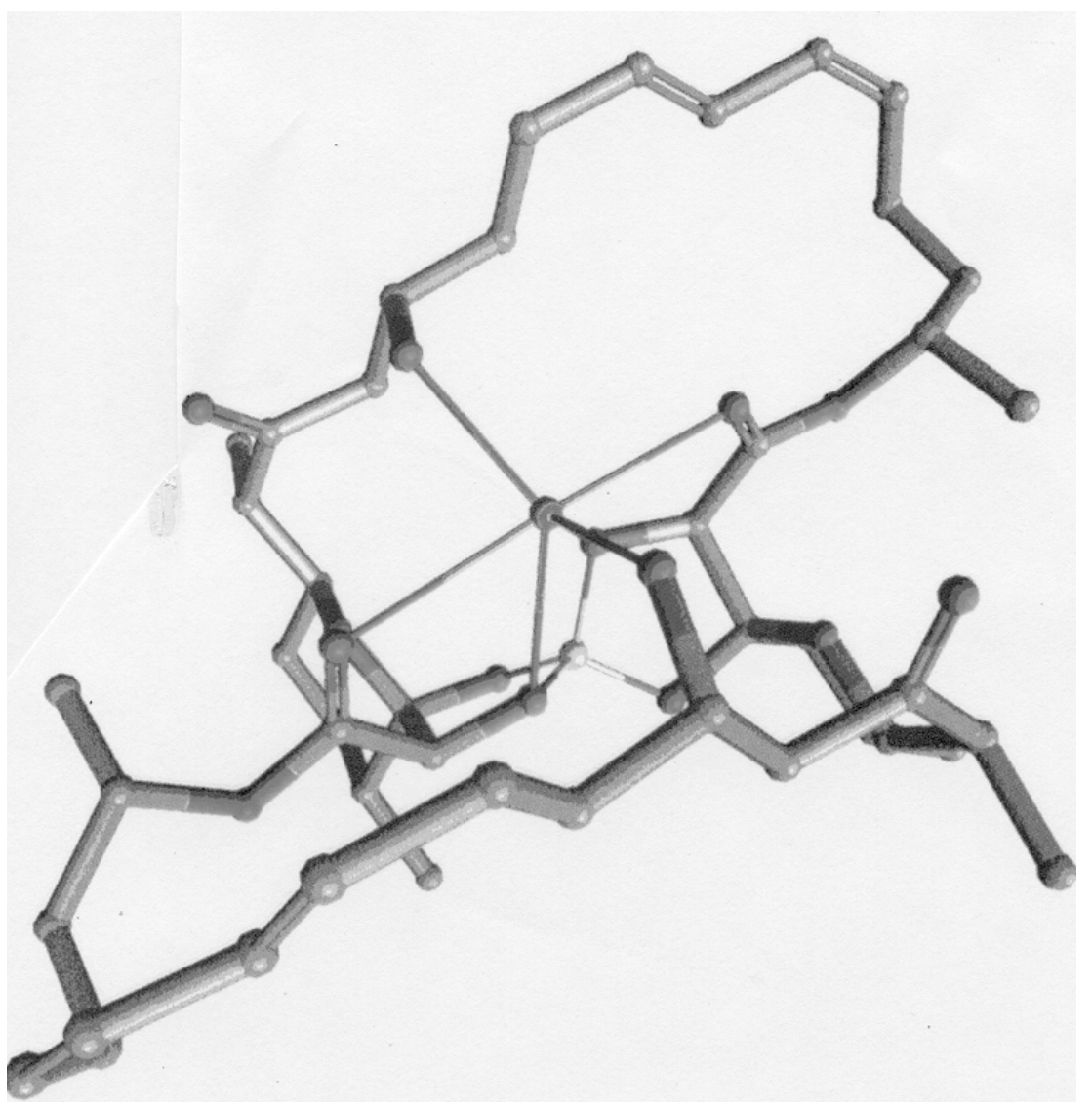


${ }^{1} \mathrm{H}-\mathrm{NMR}$ spectra of tartrolon B

$400 \mathrm{MHz}{ }^{1} \mathrm{H}$ NMR spectrum of our synthetic sample
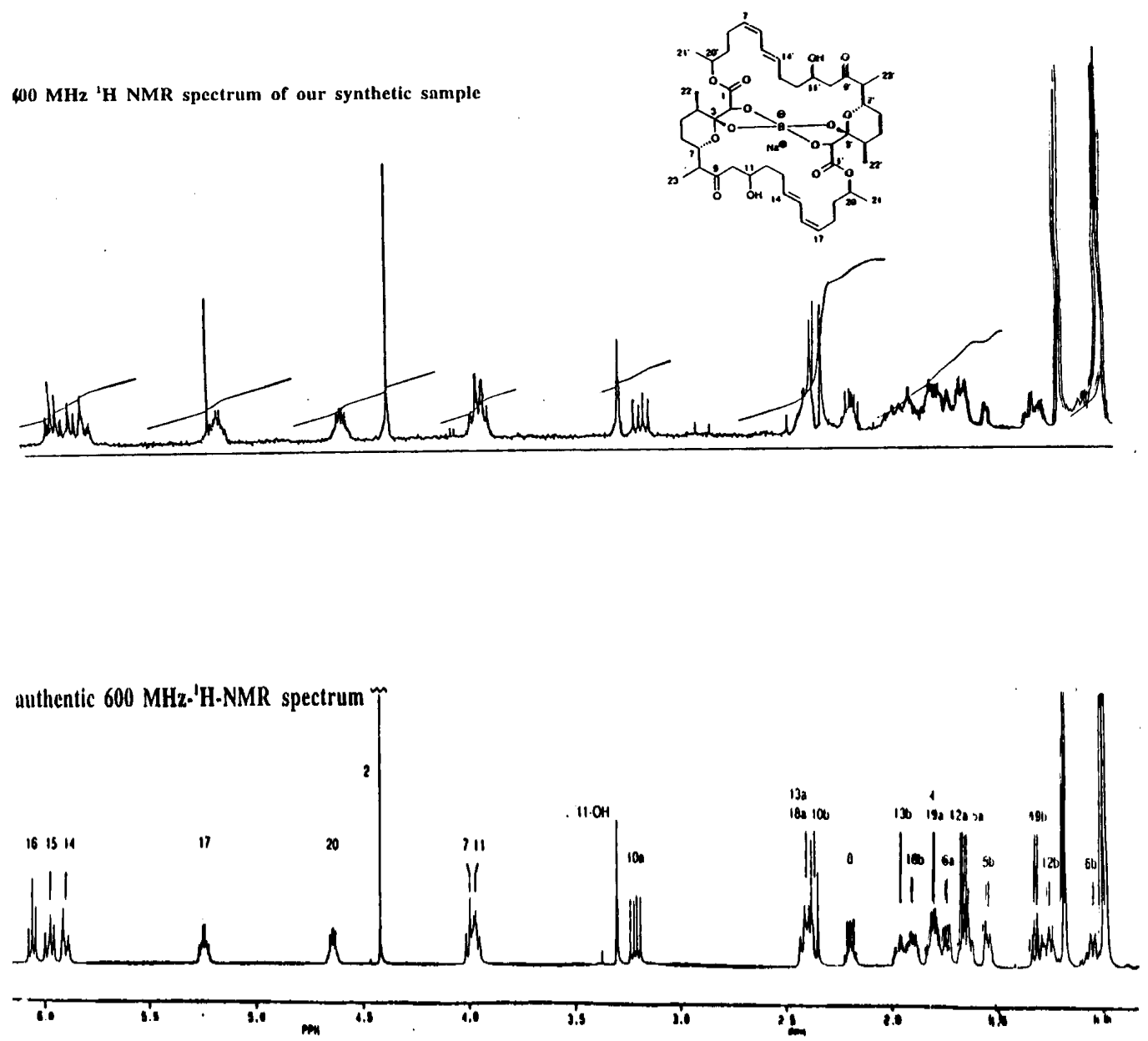


\section{${ }^{13} \mathrm{C}-\mathrm{NMR}$ spectrum of tartrolon $\mathrm{B}$}

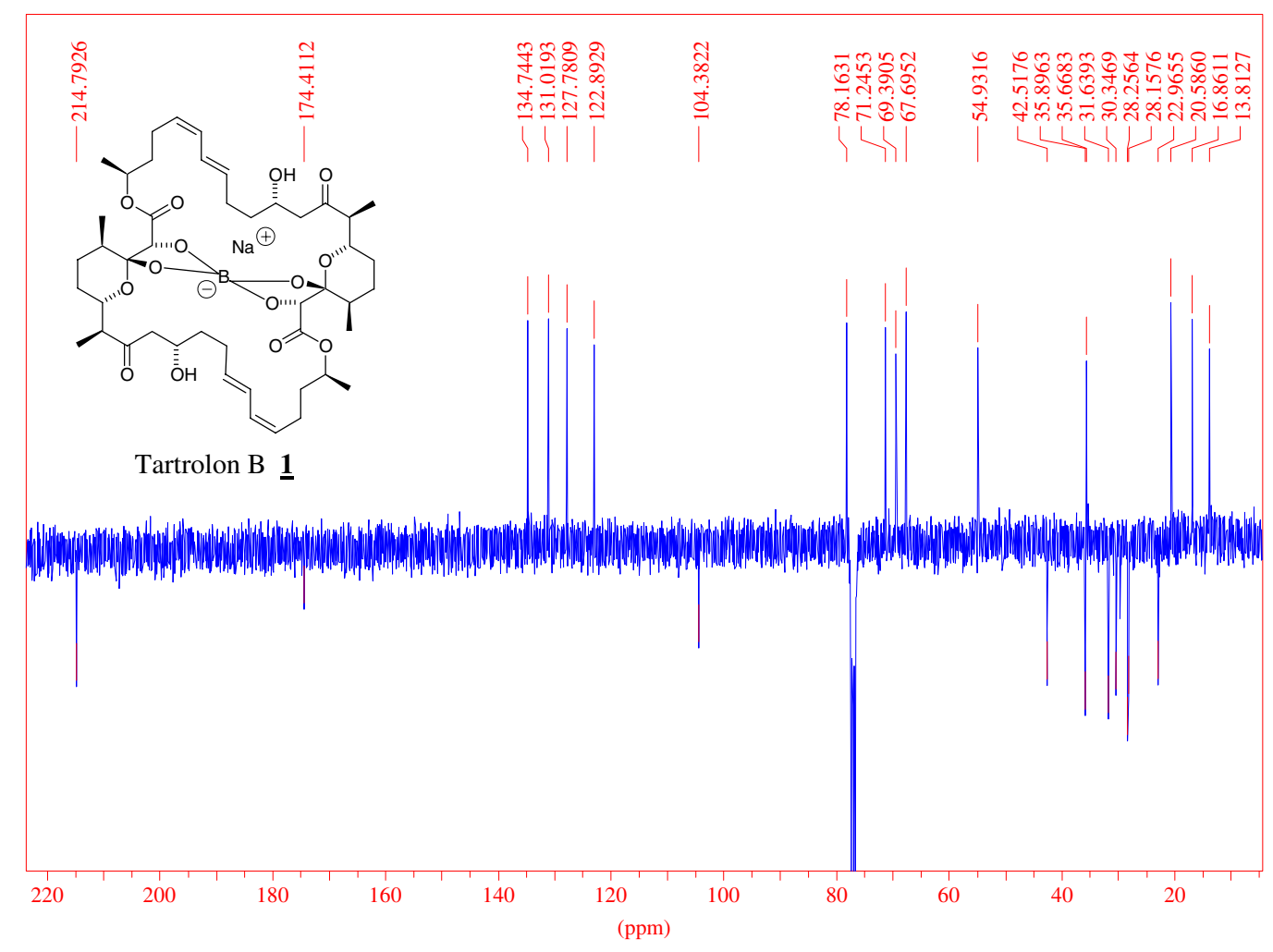



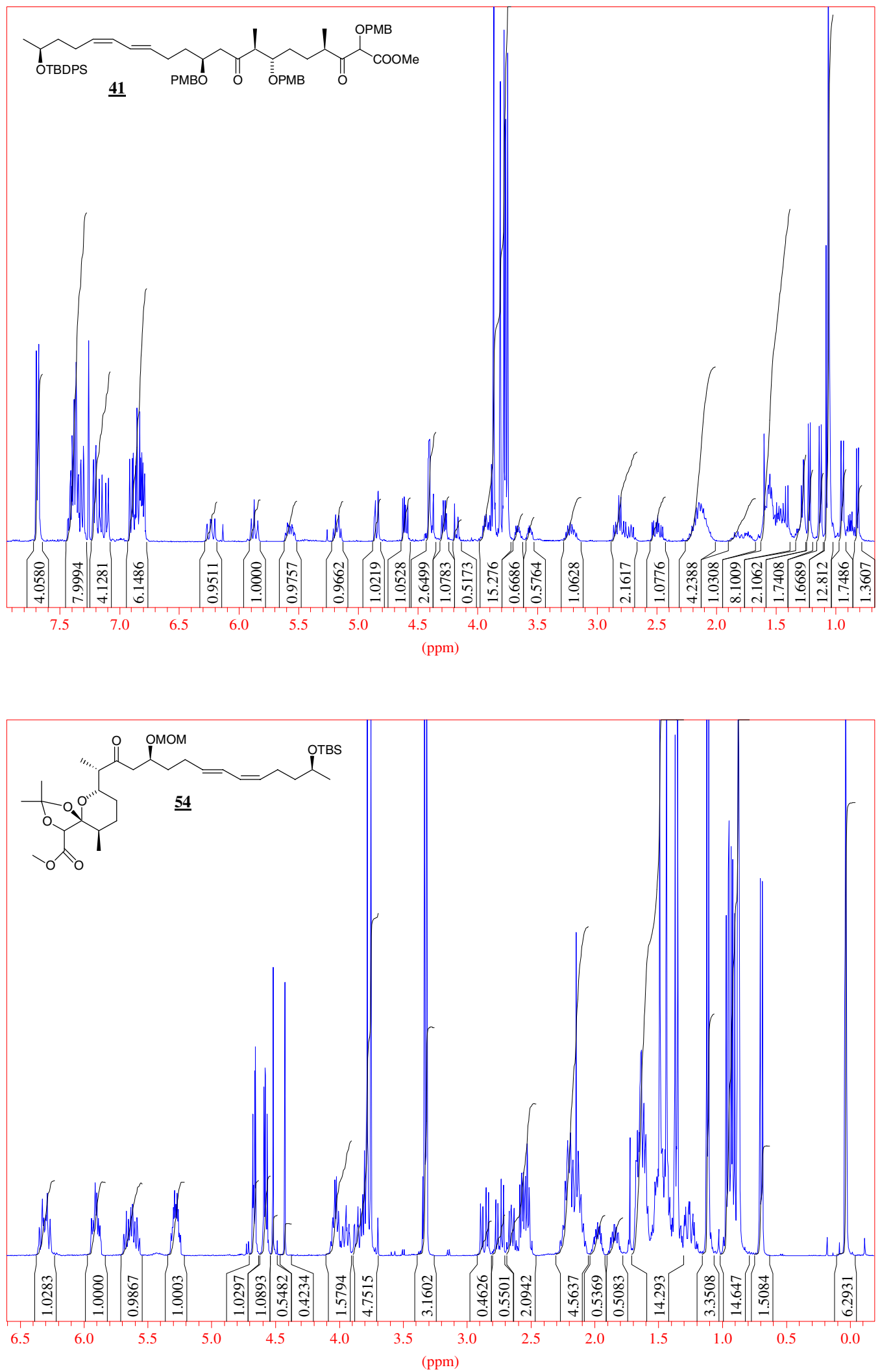